\title{
JET SCHEMES OF QUASI-ORDINARY SURFACE SINGULARITIES
}

\author{
HELENA COBO AND HUSSEIN MOURTADA
}

\begin{abstract}
In this paper we give a complete description of the irreducible components of the jet schemes (with origin in the singular locus) of a two-dimensional quasi-ordinary hypersurface singularity. We associate with these components and with their codimensions and embedding dimensions, a weighted graph. We prove that the data of this weighted graph is equivalent to the data of the topological type of the singularity. We also determine a component of the jet schemes (or equivalently, a divisor on $\mathbb{A}^{3}$ ), that computes the $\log$ canonical threshold of the singularity embedded in $\mathbb{A}^{3}$. This provides us with pairs $X \subset \mathbb{A}^{3}$ whose $\log$ canonical thresholds are not contributed by monomial divisorial valuations. Note that for a pair $C \subset \mathbb{A}^{2}$, where $C$ is a plane curve, the $\log$ canonical threshold is always contributed by a monomial divisorial valuation (in suitable coordinates of $\mathbb{A}^{2}$ ).
\end{abstract}

\section{INTRODUCTION}

A quasi-ordinary singularity $(X, 0)$ of dimension $d$, comes with a finite projection $p: X \longrightarrow \mathbb{A}^{d}$, whose discriminant is a normal crossing divisor. These singularities appear in the Jungian approach to resolution of singularities (see [35]). We are interested in irreducible quasi-ordinary hypersurfaces. Thanks to AbhyankarJung theorem, we know that a hypersurface of this type can be parametrized by a Puiseux series (i.e an element in $\left.\mathbb{C}\left[\left[x_{1}^{\frac{1}{n}}, \ldots, x_{d}^{\frac{1}{n}}\right]\right]\right)$. Moreover, some special exponents (called the characteristic exponents) which belong to the support of this series, are complete invariants of the topological type of the singularity (see [16]). In particular, they determine invariants which come from resolution of singularities, like the log canonical threshold or the Motivic zeta functions ([8], 3], [9]). They also give insights about the construction of a resolution of singularities ([6], [7, [18, [39]).

Our aim is to construct some comparable complete invariants for all type of singularities. We search for such invariants in the jet schemes. For $m \in \mathbb{N}$, the $m$-jet scheme, denoted by $X_{m}$, is a scheme that parametrizes morphisms Spec $\mathbb{C}[t] /\left(t^{m+1}\right) \longrightarrow X$. Intuitively we can think of it as parametrizing arcs in an ambient space, which have a large contact, depending on $m$, with $X$. We know already that some invariants which come from resolution of singularities are encoded in jet schemes ([32, [13]).

We want to extract from the jet schemes information about the singularity, which can be expressed in terms of invariants of resolutions of singularities. With this, our next goal is to construct a resolution of singularities by using invariants of jet schemes. For specific types of singularities, the knowledge of the irreducible components of the jet schemes $X_{m}$ of a singular variety $\mathrm{X}$, together with some invariants of them, like dimension or embedding dimension, permits to determine deep invariants of the singularity of $X$ : the topological type in the case of curves (see [26]), and the analytical type in the case of normal toric surfaces (see [27] and [28]). Moreover, in the case of irreducible plane curves, the minimal embedded resolution can be constructed from the jet schemes ([23]), and the same for rational double point singularities ([31]).

Notice that, understanding the structure of jet schemes for particular singularities, remains a difficult problem. These structures have been studied in [40] and [12] for determinantal varieties, in [26] for plane curve singularities, 27] and 28] for normal toric surfaces, in [29] for rational double point surface singularities, and in [36] for commuting matrix pairs schemes. In the toric case, no result is known for dimension bigger than two.

More general jet schemes can be defined, and there are relations with invariants of singularities (see [33]).

Date: October 14, 2018.

2010 Mathematics Subject Classification. 14E18,14J17.

Key words and phrases. Singularities, Jet schemes, Quasi-ordinary singularities, log canonical threshold. 
In this paper, we study jet schemes of a two-dimensional quasi-ordinary hypersurface singularity $X$. We will give a combinatorial description of the irreducible components of the set of $m$-jets with center in the singular locus of $X$, in terms of the following invariants of the singularity: the lattices $N_{0}, N_{1}, \ldots, N_{g}$, the minimal system of generators $\gamma_{1}, \ldots, \gamma_{g}$ of the semigroup $\Gamma$ of $X$, and the numerical data attached to them, $n_{1}, \ldots, n_{g}$ and $e_{1}, \ldots, e_{g}$ (see Section 3 for definitions). Given $h \in \mathbb{C}\left[x_{1}, \ldots, x_{n}\right]$, an algebraic variety $X$, and $p, m \in \mathbb{Z}>0$ with $p \leq m$, let Cont $_{X}^{p}(h)_{m}$ be the locally closed set defined as $\operatorname{Cont}_{X}^{p}(h)_{m}=\left\{\gamma \in X_{m} \mid \operatorname{ord}_{t}(h \circ \gamma)=p\right\}$. Then we associate with any lattice point $\nu \in N_{0}$ the constructible set

$$
D_{m}^{\nu}=\left(\operatorname{Cont}_{X}^{\nu_{1}}\left(x_{1}\right)_{m} \cap \operatorname{Cont}_{X}^{\nu_{2}}\left(x_{2}\right)_{m}\right)_{r e d},
$$

and its closure $C_{m}^{\nu}=\overline{D_{m}^{\nu}}$. The sets $C_{m}^{\nu}$ are the candidates to be the irreducible components, but there are many inclusions among them. We study these inclusions by defining, on the lattices associated with $X$, subtle relations which depend on the singular loci of the quasi-ordinary surfaces defined by the approximated roots. For some components the relation is very easy, is given by the product ordering $\leq_{p}$, and we can prove that

$$
\text { if } \nu \leq_{p} \nu^{\prime} \text { then } C_{m}^{\nu^{\prime}} \subset C_{m}^{\nu}
$$

For other components the relation is more complicated, we have that

$$
\text { if } \nu^{\prime}-\nu \in \sigma_{R e g, j^{\prime}(m, \nu)} \text { then } C_{m}^{\nu^{\prime}} \subset C_{m}^{\nu}
$$

where $j^{\prime}(m, \nu)$ is the integer $j \in\{0, \ldots, g\}$ defined by

$$
n_{j} e_{j}\left\langle\nu, \gamma_{j}\right\rangle+e_{j} \leq m<n_{j+1} e_{j+1}\left\langle\nu, \gamma_{j+1}\right\rangle+e_{j+1},
$$

and $\sigma_{R e g, j^{\prime}(m, \nu)}$ is defined to keep track of the singular locus of the $j^{\prime}(m, \nu)$-th approximated root as follows. Since the normalization of any quasi-ordinary singularity is a toric variety, let $\nu_{j}: Z_{\sigma, N_{j}} \longrightarrow V\left(f_{j}\right)$ be the normalization of $V\left(f_{j}\right)\left(f_{j}\right.$ being the $j$-th approximated root). Then $Z_{\sigma, N_{j}} \backslash \nu_{j}^{-1}\left(\operatorname{Sing}\left(V\left(f_{j}\right)\right)\right)$ is an open set of the toric variety $Z_{\sigma, N_{j}}$, it is a union of orbits, and we denote by $\sigma_{R e g, j}$ the fan formed by the faces of $\sigma$ corresponding to the orbits. Then, with the minimal elements with respect to these relations we define a set $F_{m} \subset \mathbb{Z}^{2}$, and for any $\nu \in F_{m}$, we have a component $C_{m}^{\nu} \subset X_{m}$. We prove that these are the irreducible components.

Theorem 1.1. For any $m \in \mathbb{Z}_{>0}$ we have that the space of $m$-jets of $X$ with center in the singular locus has the following decomposition into irreducible components

$$
\pi_{m}^{-1}\left(X_{\text {Sing }}\right)=\cup_{\nu \in F_{m}} C_{m}^{\nu}
$$

Hence, for every $m \in \mathbb{Z}_{>0}$, we determine the irreducible components of $X_{m}$ with center in the singular locus of $X$. Moreover, we give a formula for the codimension of $C_{m}^{\nu}$ in Proposition 4.16.

To prove the theorem we need to understand the geometry of the sets $D_{m}^{\nu}$. This is done by comparing their geometries with the geometries of the corresponding subsets for the approximate roots. The following proposition describes this comparison.

Proposition 1.2. For $m \in \mathbb{Z}_{>0}$ and $\nu \in \sigma \cap N_{j}$ such that $n_{1} \cdots n_{g}\left\langle\nu, \gamma_{1}\right\rangle \leq m$, we have that

$$
D_{m}^{\nu}=\left(\pi_{m,\left[\frac{m}{e_{j}}\right]}^{a}\right)^{-1}\left(D_{j,\left[\frac{m}{e_{j}}\right]}^{\nu}\right)
$$

where the integer $j \in\{1, \ldots, g\}$ satisfies the relation

$$
n_{j} \cdots n_{g}\left\langle\nu, \gamma_{j}\right\rangle \leq m<n_{j+1} \cdots n_{g}\left\langle\nu, \gamma_{j+1}\right\rangle
$$

by $D_{j, m}^{\nu}$ we denote the corresponding set $D_{m}^{\nu}$ for the quasi-ordinary surface defined by the $j$-th approximated root, and for $q>p, \pi_{q, p}^{a}: \mathbb{A}_{q}^{3} \longrightarrow \mathbb{A}_{p}^{3}$ is the projection of the jet schemes of the affine ambient space.

This explains in part how the singular locus of the approximated roots play a role in our problem.

The irreducible components of the jet schemes fit in natural projective systems, to which we associate a weighted graph. The vertices of the graph correspond to irreducible components, and to every vertex we attach the corresponding codimension and embedded dimension. We will prove the following result.

Theorem 1.3. The weighted graph determines and it is determined by the topological type of the singularity. 
This theorem achieves one of our goals for this type of singularities: constructing a complete invariant of the singularity from its jet schemes. Note that other invariants involving arcs and jets, like motivic zeta functions, do not determine the topological type in the case of quasi ordinary singularities, see [9] and [19].

In another direction, using Mustata's formula ([32]), we will determine an irreducible component of an $m$ jet scheme, or equivalently a divisor on the ambient space $\mathbb{A}^{3}$, which contributes the log canonical threshold of the pair $X \subset \mathbb{A}^{3}$ (note that the log canonical threshold for such a pair has been computed in [8], by looking at the poles of the motivic zeta function). This provides us with pairs $X \subseteq \mathbb{A}^{3}$ whose log canonical threshold is not contributed by a monomial divisorial valuation. For instance, for the quasi-ordinary surface defined by $f=\left(z^{2}-x_{1} x_{2}\right)^{2}-x_{1}^{3} x_{2} z$, the $\log$ canonical thresholds satisfy this property. Note that for a pair $C \subseteq \mathbb{A}^{2}$, where $C$ is a plane curve, the log canonical threshold is always contributed by a monomial valuation. See [2] and [4] for the computation of the log canonical threshold for plane curves.

Along with the same ideas of [30], we are working to construct an embedded resolution of singularities of $X$ from the data of the graph constructed in this paper. We think that such a resolution puts light on the resolution of singularities obtained by González Pérez in [18, and give in some sense an answer to the question of Lipman ([25]) on the construction of a canonical resolution of singularity of a quasi-ordinary hypersurface from the characteristic exponents. Moreover, understanding the surface case is an important step in the understanding of the general case.

The structure of the paper is as follows. In Section 2 we introduce jet schemes. A brief exposition on quasi-ordinary singularities is given in Section 3 , together with some useful definitions at the end of the section. Section 4 is the heart of the paper; it is devoted to the study of the irreducible components of the jet schemes of quasi-ordinary surface singularities. In Section 5 we state and proof some results which are useful but technical, and moreover we leave the proofs of some previous results, to make Section 4 more readable.

Acknowledgments. We thank Pedro González Pérez and the referees, for comments and suggestions which improved substantially the presentation of this paper. The beginning of this work was done during a stay of HC supported by the European Research Council under the European Community's Seventh Framework Programme (FP7/2007-2013) / ERC Grant Agreement nr. 246903 NMNAG. She thanks Institut Mathématique de Jussieu for hospitality. HM is partially supported by ANR-12-JS01-0002-01 SUSI.

\section{Jet SCHEMES}

In this section we define jet schemes of an affine scheme $X$, see $\left[20\right.$ for details. Let $X=\operatorname{Spec} \mathbb{C}\left[x_{1}, \ldots, x_{n}\right] / I$ be an affine scheme of finite type. For $m \in \mathbb{Z}_{>0}$ the functor $F_{m}: \mathbb{C}$-Schemes $\longrightarrow$ Sets which, with an affine scheme defined by a $\mathbb{C}$-algebra $A$, associates

$$
F_{m}(\operatorname{Spec}(A))=\operatorname{Hom}_{\mathbb{C}}\left(\operatorname{Spec}\left(A[t] /\left(t^{m+1}\right)\right), X\right),
$$

is representable by a $\mathbb{C}$-scheme, denoted by $X_{m}$. This is the scheme of $m$-jets. Its closed points are morphisms of the form

$$
\gamma: \operatorname{Spec}\left(\mathbb{C}[t] /\left(t^{m+1}\right)\right) \longrightarrow X
$$

Such a morphism $\gamma$ is equivalent to a $\mathbb{C}$-algebra homomorphism

$$
\gamma^{*}: \mathbb{C}\left[x_{1}, \ldots, x_{n}\right] / I \longrightarrow \mathbb{C}[t] /\left(t^{m+1}\right) .
$$

If we fix a set of generators $f_{1}, \ldots, f_{r}$ for the ideal $I$, the map $\gamma^{*}$ is determined by the image of the $x_{i}^{\prime} s$

$$
x_{i} \mapsto x_{i}^{(0)}+x_{i}^{(1)} t+\cdots+x_{i}^{(m)} t^{m}, 1 \leq i \leq n
$$

where the relations

$$
f_{i}\left(x_{1}^{(0)}+\cdots+x_{1}^{(m)} t^{m}, \ldots, x_{n}^{(0)}+\cdots+x_{n}^{(m)} t^{m}\right) \equiv 0 \bmod t^{m+1}
$$

must hold for each $f_{i}$, with $1 \leq i \leq r$. If we write equations in (11) as

$$
\begin{gathered}
f_{i}\left(x_{1}^{(0)}+x_{1}^{(1)} t+\cdots+x_{1}^{(m)} t^{m}, \ldots, x_{n}^{(0)}+x_{n}^{(1)} t+\cdots+x_{n}^{(m)} t^{m}\right)= \\
=\sum_{j=0}^{m} F_{i}^{(j)}\left(x_{1}^{(0)}, \ldots, x_{1}^{(j)}, \ldots, x_{n}^{(0)}, \ldots, x_{n}^{(j)}\right) t^{j} \bmod t^{m+1}
\end{gathered}
$$


we have that giving a closed point of $X_{m}$ is equivalent to giving a point in $V\left(F_{l}^{(j)}\right)_{0 \leq j \leq m, 1 \leq l \leq r} \subset \mathbb{A}_{m}^{n}$, where $\mathbb{A}_{m}^{n}=\operatorname{Spec}\left(\mathbb{C}\left[x_{i}^{(0)}, \ldots, x_{i}^{(m)}\right]_{i=1, \ldots, n}\right)$. Hence we can make the following identification

$$
X_{m}=\operatorname{Spec}\left(\frac{\mathbb{C}\left[x_{i}^{(0)}, \ldots, x_{i}^{(m)}\right]_{i=1, \ldots, n}}{\left(F_{l}^{(j)}\right)_{0 \leq j \leq m, 1 \leq l \leq r}}\right) .
$$

Example 2.1. Let $X$ be the quasi-ordinary surface defined by the polynomial $f=z^{3}-x_{1}^{3} x_{2}^{2}$. The equations defining the 3 -jets are

$$
\begin{aligned}
F^{(0)}= & z^{(0)^{3}}-x_{1}^{(0)^{3}} x_{2}^{(0)^{2}} \\
F^{(1)}= & 3 z^{(0)^{2}} z^{(1)}-3 x_{1}^{(0)^{2}} x_{1}^{(1)} x_{2}^{(0)^{2}}-2 x_{1}^{(0)^{3}} x_{2}^{(0)} x_{2}^{(1)} \\
F^{(2)}= & 3 z^{(0)^{2}} z^{(2)}+3 z^{(0)} z^{(1)^{2}}-6 x_{1}^{(0)^{2}} x_{1}^{(1)} x_{2}^{(0)} x_{2}^{(1)}-2 x_{1}^{(0)^{3}} x_{2}^{(0)} x_{2}^{(2)}-3 x_{1}^{(0)}{ }^{2} x_{1}^{(2)} x_{2}^{(0)}{ }^{2} \\
& -x_{1}^{(0)^{3}} x_{2}^{(1)^{2}}-3 x_{1}^{(0)} x_{1}^{(1)^{(0)}} x_{2}^{(0)} \\
F^{(3)}= & z^{(1)^{3}}+6 z^{(0)} z^{(1)} z^{(2)}+3 z^{(0)^{2}} z^{(3)}-2 x_{1}^{(0)}{ }^{3} x_{2}^{(0)} x_{2}^{(3)}-2 x_{1}^{(0)^{3}} x_{2}^{(1)} x_{2}^{(2)}-6 x_{1}^{(0)^{2}} x_{1}^{(1)} x_{2}^{(0)} x_{2}^{(2)} \\
& -3 x_{1}^{(0)} x_{1}^{(1)} x_{2}^{(1)^{2}}-6 x_{1}^{(0)^{2}} x_{1}^{(2)} x_{2}^{(0)} x_{2}^{(1)}-6 x_{1}^{(0)} x_{1}^{(1)^{2}} x_{2}^{(0)} x_{2}^{(1)}-3 x_{1}^{(0)} x_{1}^{(3)} x_{2}^{(0)^{2}} \\
& -6 x_{1}^{(0)} x_{1}^{(1)} x_{1}^{(2)} x_{2}^{(0)}{ }^{2}-x_{1}^{(1)^{3}} x_{2}^{(0)^{2}}
\end{aligned}
$$

Remark 2.2. Every polynomial $F^{(l)}$ is non-zero and quasi-homogeneous of degreel in the variables $x_{i}^{(0)}, \ldots, x_{i}^{(l)}$, $z^{(0)}, \ldots, z^{(l)}$ for $i=1,2$. In $F^{(0)}, \ldots, F^{(l)}$ the variables $x_{1}^{(l)}, x_{2}^{(l)}$ and $z^{(l)}$ appear only in $F^{(l)}$.

\section{QUASI-ORDINARY SURFACE SINGULARITIES}

In this section we collect some well known facts about quasi-ordinary hypersurface singularities of dimension two, and we prove some lemmas which will be used in the next section. We state everything for the case of dimension two, though the definitions and results hold in any dimension.

An equidimensional germ $(X, 0)$, of dimension 2, is quasi-ordinary (q.o. for short) if there exists a finite projection $p:(X, 0) \rightarrow\left(\mathbb{C}^{2}, 0\right)$ which is a local isomorphism outside a normal crossing divisor. If $(X, 0)$ is a hypersurface there is an embedding $(X, 0) \subset\left(\mathbb{C}^{3}, 0\right)$, where $X$ is defined by an equation $f=0$, and $f \in \mathbb{C}\left\{x_{1}, x_{2}\right\}[z]$ is a quasi-ordinary polynomial; that is, a Weierstrass polynomial with discriminant $\Delta_{z} f$ of the form $\Delta_{z} f=x_{1}^{\delta_{1}} \cdot x_{2}^{\delta_{2}} \epsilon$ for a unit $\epsilon$ in the ring $\mathbb{C}\left\{x_{1}, x_{2}\right\}$ of convergent power series and $\left(\delta_{1}, \delta_{2}\right) \in \mathbb{Z}_{2}^{2}$. In these coordinates the projection $p$ is the restriction of the projection

$$
\mathbb{C}^{3} \rightarrow \mathbb{C}^{2}, \quad\left(x_{1}, x_{2}, z\right) \mapsto\left(x_{1}, x_{2}\right) .
$$

From now on we assume that $(X, 0)$ is analytically irreducible, that is $f \in \mathbb{C}\left\{x_{1}, x_{2}\right\}[z]$ is irreducible (see [5] and [15] for criteria of irreducibility of q.o. polynomial). The Jung-Abhyankar theorem guarantees that the roots of a q.o. polynomial $f$, called q.o. branches, are fractional power series in $\mathbb{C}\left\{x_{1}^{1 / n}, x_{2}^{1 / n}\right\}$, for $n=\operatorname{deg} f$ (see [1]). The difference $\zeta^{(i)}-\zeta^{(j)}$ of two different roots of $f$ divides the discriminant of $f$ in the ring $\mathbb{C}\left\{x_{1}^{1 / n}, x_{2}^{1 / n}\right\}$. Therefore $\zeta^{(i)}-\zeta^{(j)}=x_{1}^{\lambda_{i j}^{(1)}} x_{2}^{\lambda_{i j}^{(2)}} u_{i j}$ where $u_{i j}$ is a unit in $\mathbb{C}\left\{x_{1}^{1 / n}, x_{2}^{1 / n}\right\}$. The exponents $\lambda_{i j}$ are characterized in the following Lemma:

Lemma 3.1. (see [16, Prop. 1.3) Let $f \in \mathbb{C}\left\{x_{1}, x_{2}\right\}[z]$ be an irreducible q.o. polynomial. Let $\zeta$ be a root of $f$ with expansion:

$$
\zeta=\sum \beta_{\lambda} \mathbf{x}^{\lambda} .
$$

There exists $0 \neq \lambda_{1}, \ldots, \lambda_{g} \in \mathbb{Q}_{\geqslant 0}^{2}$ such that $\lambda_{1} \leq \lambda_{2} \leq \cdots \leq \lambda_{g}$, and if $M_{0}:=\mathbb{Z}^{2}$ and $M_{j}:=M_{j-1}+\mathbb{Z} \lambda_{j}$ for $j=1, \ldots, g$, then:

(i) $\beta_{\lambda_{i}} \neq 0$ and if $\beta_{\lambda} \neq 0$ then $\lambda \in M_{j}$ where $j$ is the unique integer such that $\lambda_{j} \leqslant \lambda$ and $\lambda_{j+1} \not \leq \lambda$ (where $\leqslant$ means coordinate-wise and we convey that $\lambda_{g+1}=\infty$ ).

(ii) For $j=1, \ldots, g$, we have $\lambda_{j} \notin M_{j-1}$, hence the index $n_{j}=\left[M_{j-1}: M_{j}\right]$ is $>1$. 
Moreover if $\zeta \in \mathbb{C}\left\{x_{1}^{1 / n}, x_{2}^{1 / n}\right\}$ is a fractional power series satisfying the conditions above, then $\zeta$ is a quasiordinary branch.

Definition 3.2. The exponents $\lambda_{1}, \ldots, \lambda_{g}$ in Lemma 3.1 are called characteristic exponents of the q.o. branch $\zeta$. We denote by $M$ the lattice $M_{g}$ and we call it the lattice associated to the q.o. branch $\zeta$. We denote by $N$ (resp. $N_{i}$ ) the dual lattice of $M$ (resp. $M_{i}$ for $\left.i=1, \ldots, g\right)$. For convenience we denote $\lambda_{0}:=(0,0)$ and $n_{0}:=1$.

In [16] Gau proved that the characteristic exponents determine and are determined by the embedded topological type of $(X, 0)$. As a consequence of Lemma 3.1 we have the following result:

Lemma 3.3. If $\zeta$ is a quasi-ordinary branch of the form (4) then the series $\zeta_{j-1}:=\sum_{\lambda \nsucceq \lambda_{j}} \beta_{\lambda} \mathbf{x}^{\lambda}$ is a quasi-ordinary branch with characteristic exponents $\lambda_{1}, \ldots, \lambda_{j-1}$, for $j=1, \ldots, g$.

Definition 3.4. For $0 \leq j \leq g-1$ we have the germ of quasi-ordinary hypersurface $\left(X^{(j)}, 0\right)$, where $X^{(j)}$ is parametrized by the branch $\zeta_{j}$. For convenience we also denote $\zeta$ by $\zeta_{g}$ and $X$ by $X^{(g)}$.

Without loss of generality we relabel the variables $x_{1}, x_{2}$ in such a way that if $\lambda_{j}=\left(\lambda_{j}^{(1)}, \lambda_{j}^{(2)}\right) \in \mathbb{Q}^{2}$ for $j=1, \ldots, g$, then we have:

$$
\left(\lambda_{1}^{(1)}, \ldots, \lambda_{g}^{(1)}\right) \geqslant_{\text {lex }}\left(\lambda_{1}^{(2)}, \ldots, \lambda_{g}^{(2)}\right),
$$

where $\geqslant_{\text {lex }}$ is lexicographic order. The q.o. branch $\zeta$ is said to be normalized if $\lambda_{1}$ is not of the form $\left(\lambda_{1}^{(1)}, 0\right)$ with $\lambda_{1}^{(1)}<1$. Lipman proved that the germ $(X, 0)$ can be parametrized by a normalized q.o. branch (see [16], Appendix). We assume from now on that the q.o. branch $\zeta$ is normalized.

The semigroup $\mathbb{Z}_{\geqslant 0}^{2}$ has a minimal set of generators $v_{1}, v_{2}$, which is a basis of the lattice $M_{0}$. The dual basis, $\left\{w_{1}, w_{2}\right\}$, is a basis of the dual lattice $N_{0}$, and spans a regular cone $\sigma$ in $N_{0, \mathbb{R}}=N_{0} \otimes_{\mathbb{Z}} \mathbb{R}$. It follows that $\mathbb{Z}_{\geqslant 0}^{2}=\sigma^{\vee} \cap M_{0}$, where $\sigma^{\vee}=\mathbb{R}_{\geqslant 0}^{2}$ is the dual cone of $\sigma$. The $\mathbb{C}$-algebra $\mathbb{C}\left\{x_{1}, x_{2}\right\}$ is isomorphic to $\mathbb{C}\left\{\sigma^{\vee} \cap M_{0}\right\}$. The local algebra $\mathcal{O}_{X}=\mathbb{C}\left\{x_{1}, x_{2}\right\}[z] /(f)$ of the singularity $(X, 0)$ is isomorphic to $\mathbb{C}\left\{\sigma^{\vee} \cap M_{0}\right\}[\zeta]$. By Lemma 3.1 the series $\zeta$ can be viewed as an element $\sum \beta_{\lambda} \mathbf{x}^{\lambda}$ of the algebra $\mathbb{C}\left\{\sigma^{\vee} \cap M\right\}$.

Lemma 3.5. (see [18]) The homomorphism $\mathcal{O}_{X} \longrightarrow \mathbb{C}\left\{\sigma^{\vee} \cap M\right\}$ is the inclusion of $\mathcal{O}_{X}$ in its integral closure in its field of fractions.

This Lemma shows that the normalization of a quasi-ordinary hypersurface $(X, 0)$ is the germ of the toric variety $X(\sigma, N)=Z^{\sigma^{\vee} \cap M}$ at the distinguished point.

The elements of $M$ defined by:

$$
\gamma_{1}=\lambda_{1} \text { and } \gamma_{j+1}-n_{j} \gamma_{j}=\lambda_{j+1}-\lambda_{j} \text { for } j=1, \ldots, g-1 \text {, }
$$

span the semigroup $\Gamma:=\mathbb{Z}_{\geqslant 0}^{2}+\gamma_{1} \mathbb{Z}_{\geqslant 0}+\cdots+\gamma_{g} \mathbb{Z}_{\geqslant 0} \subset \sigma^{\vee} \cap M$. For convenience we denote $\gamma_{0}:=0$. The semigroup $\Gamma$ defines an analytic invariant of the germ $(X, 0)$ (see [17], [34, 222]).

Definition 3.6. The monomial variety associated to $(X, 0)$ is the toric variety

$$
X^{\Gamma}:=\operatorname{Spec} \mathbb{C}[\Gamma] \text {. }
$$

Moreover we associate with the characteristic exponents the following sequence of semigroups:

$$
\Gamma_{j}=\sigma^{\vee} \cap M+\gamma_{1} \mathbb{Z}_{\geq 0}+\cdots+\gamma_{j} \mathbb{Z}_{\geq 0}, \text { for } j=0, \ldots, g .
$$

And we have the corresponding monomial varieties associated to $\Gamma_{j}$. We denote by $e_{i-1}:=n_{i} \cdots n_{g}$ for $1<i \leq g$ and set $e_{g}:=1$. Notice that, by (5) and the definition of $\gamma_{1}, \ldots, \gamma_{g}$, we deduce that

$$
\left(\gamma_{1}^{(1)}, \ldots, \gamma_{g}^{(1)}\right) \geqslant_{\text {lex }}\left(\gamma_{1}^{(2)}, \ldots, \gamma_{g}^{(2)}\right) .
$$

The following Lemma gathers some important facts about the generators $\gamma_{j}$ and the semigroups $\Gamma_{j}$.

Lemma 3.7. (see Lemma 3.3 in [17])

(i) We have that $\gamma_{j}>n_{j-1} \gamma_{j-1}$ for $j=2, \ldots, g$, where $<$ means $\neq$ and $\leq$ coordinate-wise.

(ii) If a vector $u_{j} \in \sigma^{\vee} \cap M_{j}$, then we have $u_{j}+n_{j} \gamma_{j} \in \Gamma_{j}$. 
(iii) The vector $n_{j} \gamma_{j}$ belongs to the semigroup $\Gamma_{j-1}$ for $j=1, \ldots, g$. Moreover, we have a unique relation

$$
n_{j} \gamma_{j}=\alpha^{(j)}+r_{1}^{(j)} \gamma_{1}+\cdots+r_{j-1}^{(j)} \gamma_{j-1}
$$

such that $0 \leq r_{i}^{(j)} \leq n_{i}-1$ and $\alpha^{(j)} \in M_{0}$ for $j=1, \ldots, g$.

Definition 3.8. Given two irreducible quasi-ordinary polynomials $f$ and $g$ in $\mathbb{C}\left\{x_{1}, x_{2}\right\}[z]$ such that $f g$ is a quasi-ordinary polynomial, we say that $f$ and $g$ have order of coincidence $\alpha \in \mathbb{Q}^{2}$ if $\alpha$ is the largest exponent on the set

where $\zeta^{(i)}$ and $\zeta^{(j)}$ are roots of $f g$.

$$
\left\{\lambda_{i j} \mid f\left(\zeta^{(i)}\right)=g\left(\zeta^{(j)}\right)=0\right\}
$$

Definition 3.9. We associate to $f$ a set of semi-roots

$$
z=f_{0}, f_{1} \ldots, f_{g}=f \in \mathbb{C}\left\{x_{1}, x_{2}\right\}[z] .
$$

Every $f_{j}$ is an irreducible quasi-ordinary polynomial of degree $n_{0} \cdots n_{j}$ with order of coincidence with $f$ equal to $\lambda_{j+1}$ for $j=0, \ldots, g$.

They are parametrized by truncations of a root $\zeta\left(x_{1}^{1 / n}, x_{2}^{1 / n}\right)$ of $f$ in the following sense:

Proposition 3.10. (see [17]) Let $q \in \mathbb{C}\left\{x_{1}, x_{2}\right\}[z]$ be a monic polynomial of degree $n_{0} \cdots n_{j}$. Then $q$ is a $j$-th semi-root of $f$ if and only if $q(\zeta)=\mathbf{x}^{\gamma_{j}} \epsilon_{j}$ for a unit $\epsilon_{j}$ in $\mathbb{C}\left\{x_{1}, x_{2}\right\}[z]$.

Corollary 3.11. The quasi-ordinary polynomials $f_{j} \in \mathbb{C}\left\{x_{1}, x_{2}\right\}[z]$ defining $X^{(j)}$ (see Definition 3.4) for $j=0, \ldots, g$ form a system of semiroots of $f$.

In what follows we state some results about quasi-ordinary polynomials and approximated roots. Moreover we give some definitions and notations that will be used in the next section.

Approximated roots play an important role in the understanding of quasi-ordinary singularities. We have the following expansions of the semiroots in terms of the previous ones:

Lemma 3.12. (See Lemma 35 in [18]) The expansion of the approximated roots is of the following form:

$$
c_{j}^{*} f_{j}=f_{j-1}^{n_{j}}-c_{j} x_{1}^{\alpha_{1}^{(j)}} x_{2}^{\alpha_{2}^{(j)}} f_{0}^{r_{1}^{(j)}} \cdots f_{j-2}^{r_{j-1}^{(j)}}+\sum c_{\underline{\alpha}, \underline{r}} x_{1}^{\alpha_{1}} x_{2}^{\alpha_{2}} f_{0}^{r_{1}} \cdots f_{j-1}^{r_{j}},
$$

where $c_{j}^{*}, c_{j} \in \mathbb{C}^{*}, 0 \leq r_{i}^{(j)}, r_{i}<n_{i}$ for $i=1, \ldots, j$, and

$$
n_{j} \gamma_{j}=\left(\alpha_{1}^{(j)}, \alpha_{2}^{(j)}\right)+r_{1}^{(j)} \gamma_{1}+\cdots+r_{j-1}^{(j)} \gamma_{j-1}<\left(\alpha_{1}, \alpha_{2}\right)+r_{1} \gamma_{1}+\cdots+r_{j} \gamma_{j}
$$

Let $(X, 0) \subset\left(\mathbb{C}^{3}, 0\right)$ be a germ of quasi-ordinary surface with characteristic exponents $\lambda_{1}, \ldots, \lambda_{g}$. We denote by $\lambda_{1}=\left(\frac{a_{1}}{n_{1}}, \frac{b_{1}}{n_{1}}\right)$ the first characteristic exponent. Notice that, by (5), we have that $a_{1} \geq b_{1} \geq 0$.

Lemma 3.13. We have that

$$
f_{1}=z^{n_{1}}-c_{1} x_{1}^{a_{1}} x_{2}^{b_{1}}+\sum c_{i j k} x_{1}^{i} x_{2}^{j} z^{k},
$$

with $(i, j)+k \gamma_{1}>n_{1} \gamma_{1}$ and $k<n_{1}$ whenever $c_{i j k} \neq 0$. And for $1 \leq l \leq g-1$ we have

$$
f=f_{l}^{e_{l}}+\sum_{(i, j)+k \gamma_{1}>n_{l} e_{l} \gamma_{l}} c_{i j k}^{(l)} x_{1}^{i} x_{2}^{j} z^{k} .
$$

Moreover, the following expansions will be useful. For $0 \leq j<g-1$

$$
f=f_{j}^{e_{j}}+\sum c_{\underline{\alpha}, \underline{r}} x_{1}^{\alpha_{1}} x_{2}^{\alpha_{2}} z^{r_{1}} \cdots f_{j}^{r_{j+1}}
$$

where $\left(\alpha_{1}, \alpha_{2}\right)+r_{1} \gamma_{1}+\cdots+r_{j+1} \gamma_{j+1}>n_{j+1} e_{j+1} \gamma_{j+1}$ whenever $c_{\underline{\alpha}, \underline{r}} \neq 0$.

$$
f=f_{j}^{e_{j}}+\sum c_{\underline{\alpha}, \underline{r}} x_{1}^{\alpha_{1}} x_{2}^{\alpha_{2}} z^{r_{1}} \cdots f_{j-1}^{r_{j}}
$$

where $\left(\alpha_{1}, \alpha_{2}\right)+r_{1} \gamma_{1}+\cdots+r_{j} \gamma_{j}>n_{j} e_{j} \gamma_{j}$ whenever $c_{\underline{\alpha}, \underline{r}} \neq 0$.

Proof. By applying recursively Lemma 3.12 and using Lemma 3.7 (i). 
3.1. Some toric geometry. See [14 for a reference on toric geometry.

Given a lattice $N$ we denote by $N_{\mathbb{R}}$ the vector space spanned by $N$ over the field $\mathbb{R}$. We denote by $M$ the dual lattice, $M=\operatorname{Hom}(N, \mathbb{Z})$, and by $\langle\rangle:, N \times M \longrightarrow \mathbb{Z}$ the duality pairing between the lattices $N$ and $M$. A rational convex polyhedral cone (or simply a cone) is the set of non-negative linear combinations of vectors $v_{1}, \ldots, v_{r} \in N$. A cone is strictly convex if it contains no lines. The dual cone of $\sigma$, denoted by $\sigma^{\vee}$, is the set

$$
\sigma^{\vee}=\left\{\omega \in M_{\mathbb{R}} \mid\langle\omega, u\rangle \geq 0 \forall u \in \sigma\right\},
$$

and the orthogonal of $\sigma$, denoted by $\sigma^{\perp}$, is

$$
\sigma^{\perp}=\left\{\omega \in M_{\mathbb{R}} \mid\langle\omega, u\rangle=0 \forall u \in \sigma\right\} .
$$

We denote by $\stackrel{\circ}{\sigma}$ the relative interior of the cone $\sigma$. A fan $\Sigma$ is a family of strictly convex cones in $N_{\mathbb{R}}$ such that for any $\sigma \in \Sigma$ any face of $\sigma$ belongs to $\Sigma$, and for any $\sigma, \tau \in \Sigma$, the intersection $\sigma \cap \tau$ is a face of both. The relation $\tau \leq \sigma$ denotes that $\tau$ is a face of $\sigma$. The support of the fan $\Sigma$ is the set $|\Sigma|:=\cup_{\tau \in \Sigma} \tau \subset N_{\mathbb{R}}$.

Let $\tau$ be a strictly convex cone, rational for the lattice $N$. By Gordan's Lemma the semigroup $\sigma^{\vee} \cap M$ is finitely generated. We denote by $\mathbb{C}\left[\sigma^{\vee} \cap M\right]$ the semigroup algebra of $\sigma^{\vee} \cap M$ with complex coefficients. The toric variety $Z(\tau, N):=\operatorname{Spec} \mathbb{C}\left[\tau^{\vee} \cap M\right]$ is normal. The torus $T_{N}:=Z(N)$ is an open dense subset of $Z(\tau, N)$ which acts on $Z(\tau, N)$ and the action extends the action of the torus on itself by multiplication. There is a one to one correspondence between the faces $\theta$ of $\tau$ and the orbits $\operatorname{orb}(\theta)$ of the torus action on $Z(\tau, N)$, which reverses the inclusions of their closures. The closure of $\operatorname{orb}(\theta)$ is the toric variety $Z\left(\left(\sigma^{\vee} \cap \theta^{\perp}\right)^{\vee}, N\right)$ for $\tau \leq \sigma$.

3.2. Definitions and Notations. We introduce now some definitions and notations which will be used throughout the paper.

The singular locus of a quasi-ordinary singularity is determined, after Lipman, by its characteristic exponents (see [24] and [34]).

Definition 3.14. We define

$$
\begin{aligned}
& Z_{i}=X \cap\left\{x_{i}=0\right\}, \quad \text { for } i=1,2 \\
& Z_{12}=X \cap\left\{x_{1}=x_{2}=0\right\} .
\end{aligned}
$$

Moreover, the smallest number $c \in\{1,2\}$ with the property that

$$
\lambda_{i}^{(j)}=0, \text { for all } 1 \leq i \leq g \text { and } c+1 \leq j \leq 2
$$

is called the equisingular dimension of the quasi-ordinary projection $p$.

In 24] Lipman proved that the spaces $Z_{1}, Z_{2}$ and $Z_{12}$ are irreducible. By condition (5) we have that $c$ gives the number of variables appearing in the monomials $\mathbf{x}^{\lambda_{1}}, \ldots, \mathbf{x}^{\lambda_{g}}$.

Definition 3.15. Let $X$ be a quasi-ordinary surface singularity with $g \geq 1$ characteristic exponents. We define the integers $g_{1} \geq 0$ and $g_{2} \in\left\{g_{1}, g_{1}+1\right\}$ as follows

$$
\text { if } c=1 \text { we set } g_{1}=g_{2}=g+1 \text {, }
$$

otherwise

$$
\begin{aligned}
& \gamma_{g_{1}}^{(2)}=0 \text { and } \gamma_{g_{1}+1}^{(2)} \neq 0, \\
& g_{2}=\left\{\begin{array}{cc}
g_{1}+1 & \text { if } \gamma_{g_{1}+1}^{(2)}=\frac{1}{n_{g_{1}+1}} \\
g_{1} & \text { otherwise }
\end{array}\right.
\end{aligned}
$$

Note that these integers can be defined with the same property for the characteristic exponents. Now we use them to describe the singular locus of $X$. Lipman's theorem describes the singular locus $X_{\text {Sing }}$ of a quasi-ordinary hypersurface $X$. We state it here in the particular case of surfaces.

Theorem 3.16. (See Theorem 7.3 in [24]) Let $X$ be a quasi-ordinary surface singularity with characteristic exponents $\lambda_{1}, \ldots, \lambda_{g}$. Then we have:

(i) $X_{\text {Sing }}=Z_{12}$ if and only if $g=1$ and $\lambda_{1}=\left(\frac{1}{n}, \frac{1}{n}\right)$.

(ii) If $c=1$ then $X_{\text {Sing }}=Z_{1}$. 
(iii) Otherwise $c=2$, and since $\lambda_{1}^{(1)} \neq 0, Z_{1} \subset X$ is a component of $X_{\text {Sing. Moreover, if we do not have }}$ simultaneously $\lambda_{k}^{(2)}=0$ for all $1 \leq k \leq g-1$ and $\lambda_{g}^{(2)}=\frac{1}{n_{g}}$ then the singular locus is reducible of the form $X_{\text {Sing }}=Z_{1} \cup Z_{2}$.

Remark 3.17. Notice that

$$
\begin{aligned}
& Z_{1}=\left\{x_{1}=z=0\right\} \\
& Z_{2}=\left\{x_{2}=f_{g_{1}}=0\right\} \\
& Z_{12}=\{(0,0,0)\}
\end{aligned}
$$

and hence the singular locus of a quasi-ordinary surface singularity is either a point, or a line, or two lines, or a line and a singular curve.

Then, geometrically, the meaning of the integer $g_{2}$ is to measure the irreducibility of the singular locus of the approximated roots. Indeed,

$$
\begin{aligned}
& X_{\text {Sing }}^{(j)} \text { is irreducible, for } 1 \leq j \leq g_{2} \\
& X_{\text {Sing }}^{(j)} \text { is reducible, for } g_{2}<j \leq g
\end{aligned}
$$

Now we define a sequence of semi-open cones keeping track of the singular locus of the quasi-ordinary hypersurfaces $X^{(j)}$ for $j=1, \ldots, g$ (see Definition 3.4). Let

$$
\nu_{j}: X\left(\sigma, N_{j}\right) \longrightarrow X^{(j)}
$$

be the normalization of $X^{(j)}$ (see Lemma 3.5). Consider $\nu_{j}^{-1}\left(X_{\text {Sing }}^{(j)}\right) \subseteq X\left(\sigma, N_{j}\right)$, it is a disjoint union of orbits

$$
\nu_{j}^{-1}\left(X_{\text {Sing }}^{(j)}\right)=\bigsqcup_{\tau} \operatorname{orb}(\tau), \text { for some } \tau \text { faces of } \sigma \text {. }
$$

We also have that the complement of $\nu_{j}^{-1}\left(X_{\text {Sing }}^{(j)}\right)$ in the toric variety $X\left(\sigma, N_{j}\right)$ is a union of orbits.

Definition 3.18. We define, for $j=1, \ldots, g$

$$
\begin{aligned}
& \sigma_{\text {Sing }, j} \text { the fan associated to } \nu_{j}^{-1}\left(X_{\text {Sing }}^{(j)}\right) \\
& \sigma_{\text {Reg, } j} \text { the fan associated to } X\left(\sigma, N_{j}\right) \backslash \nu_{j}^{-1}\left(X_{\text {Sing }}^{(j)}\right)
\end{aligned}
$$

For $j=g$ we just denote them by $\sigma_{\text {Sing }}$ and $\sigma_{\text {Reg }}$ respectively.

Remark 3.19. Recall that $\sigma=\mathbb{R}_{\geq 0}^{2}$, let $\rho_{1}$ and $\rho_{2}$ be its one-dimensional faces. For $1 \leq j \leq g$ we have

$$
\sigma_{\text {Sing }, j}=\left\{\begin{array}{ccc}
\sigma \backslash\left(\rho_{1} \cup \rho_{2}\right) & \text { if } & X_{\text {Sing }}^{(j)}=Z_{12} \\
\sigma \backslash \rho_{2} & \text { if } & X_{\text {Sing }}^{(j)}=Z_{1} \\
\sigma \backslash\{(0,0)\} & \text { if } & X_{\text {Sing }}^{(j)}=Z_{1} \cup Z_{2}
\end{array}\right.
$$

and by definition $\sigma_{\text {Reg,j }}=\sigma \backslash \sigma_{\text {Sing }, j}$.

The fan $\sigma_{\text {Sing }}$ will turn out to be necessary in our description of $\pi_{m}^{-1}\left(X_{\text {Sing }}\right)$ (see Lemma 4.2), while the fans $\sigma_{R e g, j}$ will be important in the description of the irreducible components (see Proposition 4.21).

The sequence $\left\{\sigma_{R e g, 1}, \ldots, \sigma_{R e g, g}\right\}$ is not very complicated, in the sense that most of the elements are the same. Since by definition $\gamma_{g_{1}+1}^{(2)}=\lambda_{g_{1}+1}^{(2)}$ then, by Theorem 3.16, we deduce

$$
\begin{aligned}
& \text { for } 1 \leq j \leq g_{2} \quad \sigma_{\text {Reg }, j}=\left\{\begin{array}{cl}
\rho_{1} \cup \rho_{2} & \text { if } \gamma_{1}=\left(\frac{1}{n_{1}}, \frac{1}{n_{1}}\right) \text { and } j=1 \\
\rho_{2} & \text { otherwise }
\end{array}\right. \\
& \text { for } g_{2}+1 \leq j \leq g \quad \sigma_{\text {Reg }, j}=\{(0,0)\}
\end{aligned}
$$

Moreover notice that, by definition, we have $\sigma_{\text {Sing }, j} \subseteq \sigma_{\text {Sing }, j+1}$. 
Definition 3.20. Given $\nu \in \sigma \cap N_{0}$, we define the following sequence of real numbers

$$
l_{i}(\nu):=n_{i} e_{i}\left\langle\nu, \gamma_{i}\right\rangle \text {, for } 1 \leq i \leq g .
$$

Set $l_{0}(\nu)=0$ and $l_{g+1}(\nu)=\infty$ for any $\nu \in \mathbb{Z}^{2}$. Moreover, we define

$$
i(\nu)=\left\{\begin{array}{cl}
g+1 & \text { if } \nu \in N_{g} \\
\min \left\{i \in\{1, \ldots, g\} \mid \nu \notin N_{i}\right\} & \text { otherwise }
\end{array}\right.
$$

and

$$
\begin{aligned}
& \left.c(\nu)=\max \{0 \leq i \leq g\} \mid\left\langle\nu, \gamma_{i}\right\rangle=0\right\} \\
& m(\nu)=\min \left\{1 \leq i \leq g \mid n_{i}\left\langle\nu, \gamma_{i}\right\rangle<\left\langle\nu, \gamma_{i+1}\right\rangle\right\}
\end{aligned}
$$

Notice that, by definition, $l_{1}(\nu)=l_{2}(\nu)=\cdots=l_{m(\nu)}(\nu)<l_{m(\nu)+1}(\nu)$. Moreover, for $\nu \in \sigma_{S i n g} \cap N_{0}$, we have that

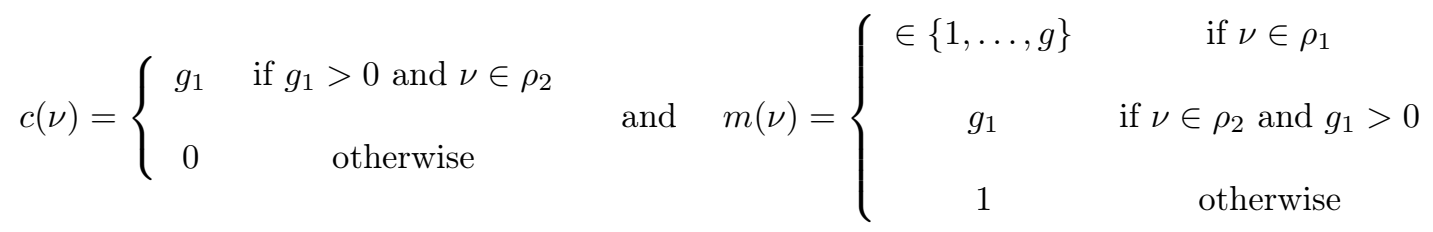

It is straightforward to check that $c(\nu) \leq m(\nu) \leq i(\nu)$.

Lemma 3.21. For any $\nu \in \sigma_{\text {Sing }} \cap N_{0}$, we have that

$$
l_{i}(\nu) \in \mathbb{Z} \text { for } 1 \leq i \leq \min \{i(\nu), g\} .
$$

Moreover the integers $l_{i}(\nu)$ are ordered as

$$
0=l_{c(\nu)}(\nu)<l_{c(\nu)+1}(\nu) \leq \cdots \leq l_{g}(\nu)<l_{g+1}(\nu)=\infty,
$$

and we have the equality $l_{i}(\nu)=l_{i+1}(\nu)$ if and only if $\gamma_{i+1}^{(j)}=n_{i} \gamma_{i}^{(j)}$ for $j$ either 1 or 2 , and $\nu \in \rho_{j}$.

For $i>c(\nu)$ we have that the following statements are equivalent:

(i) $\nu \in N_{i}$

(ii) for $1 \leq j \leq i-1, \nu \in N_{j}$ and the number $\frac{l_{j+1}(\nu)-l_{j}(\nu)}{e_{j}}$ is a positive integer.

Proof. For $1 \leq i<i(\nu)$ we have that $\nu \in N_{i}$, therefore $\left\langle\nu, \gamma_{i}\right\rangle \in \mathbb{Z}$ and $l_{i}(\nu)$ is an integer. If $i(\nu)<g+1$, then $\nu \in N_{i(\nu)-1}$ and by (8) we deduce that $l_{i(\nu)}(\nu)$ is an integer.

By definition of $\gamma_{i}$ it follows that $e_{i} \gamma_{i+1}=e_{i-1} \gamma_{i}+e_{i}\left(\lambda_{i+1}-\lambda_{i}\right)$. Then

$$
l_{i+1}(\nu)=l_{i}(\nu)+e_{i}\left\langle\nu, \lambda_{i+1}-\lambda_{i}\right\rangle
$$

and the second claim of the Lemma follows because the exponents $\lambda_{i}$ are ordered lexicographically as (5).

The equivalence follows by the fact that

$$
l_{j}(\nu)-l_{j-1}(\nu)=e_{j-1}\left(\left\langle\nu, \gamma_{j}\right\rangle-n_{j-1}\left\langle\nu, \gamma_{j-1}\right\rangle\right) .
$$

\section{JET SCHEMES OF QUASI-ORDINARY SURFACE SINGULARITIES}

In this section we describe the irreducible components of $\pi_{m}^{-1}\left(X_{S i n g}\right) \subset X_{m}$. We begin with an overview of the section.

We will associate, to any $\nu=\left(\nu_{1}, \nu_{2}\right) \in \sigma_{\text {Sing }} \cap N_{0}$ with $0 \leq \nu_{i} \leq m$, a family of $m$-jets that we call $C_{m}^{\nu}$. Roughly speaking, it is the Zariski closure of the set of $m$-jets whose order of contact with the hyperplane coordinate $x_{i}$ is bigger or equal to $\nu_{i}$, for $i=1,2$. We divide the sets $C_{m}^{\nu}$ into two types. Sets defined by the annihilation of hyperplane coordinates in $\mathbb{A}_{m}^{3}=\operatorname{Spec} \mathbb{C}\left[x_{1}^{(i)}, x_{2}^{(i)}, z^{(i)}\right]_{i=0, \ldots, m}$, which are associated with $\nu$ in a certain set $H_{m} \subset \sigma_{\text {Sing }} \cap N_{0}$ (where $H$ stands for hyperplane). The $C_{m}^{\nu}$ of the second type have a more complicated geometry and are associated with $\nu$ in a certain set $L_{m}$ (where $L$ stands for lattice, because for such components, $\nu$ belongs to one of the lattices $N_{i}$ for $1 \leq i \leq g$ ).

The geometry of $C_{m}^{\nu}$ for $\nu \in H_{m}$ is evident and the possible inclusions among different $C_{m}^{\nu}$ are determined only by looking at the product ordering of the associated vectors $\nu$. For $\nu \in L_{m}$, to understand the geometry 
of $C_{m}^{\nu}$, we consider the order of contact of the generic point of $C_{m}^{\nu}$ with the different approximated roots. This allows us to detect certain dense subset of $C_{m}^{\nu}$, which is isomorphic to the cartesian product of an open set of the spectrum of the graded algebra (associated with the last approximated root appearing in the equations defining $C_{m}^{\nu}$ ) and an affine space. This permits to prove that each $C_{m}^{\nu}$ is irreducible and to compute its codimension (see Proposition 4.16).

The inclusions among the $C_{m}^{\nu}$ for $\nu \in H_{m} \cup L_{m}$ are more delicate. We introduce in Definition 4.22 a new relation to detect such inclusions. In this definition, the singular locus of the last approximated root affecting the geometry of $C_{m}^{\nu}$, plays a crucial role. Finally, with the collection of sets $C_{m}^{\nu}$ left, we prove in Theorem 4.24 that they are the irreducible components of the $m$-jets through the singular locus.

Definition 4.1. Let $h \in \mathbb{C}\left[x_{1}, \ldots, x_{n}\right]$ and let $X$ be an algebraic variety. For $p, m \in \mathbb{Z}_{>0}$ with $p \leq m$ we set

$$
\operatorname{Cont}_{X}^{p}(h)_{m}:=\left\{\gamma \in X_{m} \mid \operatorname{ord}_{t}(h \circ \gamma)=p\right\} .
$$

And, for $m \in \mathbb{Z}_{>0}$ and any $\nu=\left(\nu_{1}, \nu_{2}\right) \in \sigma_{\text {Sing }} \cap N_{0}$ with $\nu_{i} \leq m$ we define the constructible set

$$
D_{m}^{\nu}=\left(\operatorname{Cont}_{X}^{\nu_{1}}\left(x_{1}\right)_{m} \cap \operatorname{Cont}_{X}^{\nu_{2}}\left(x_{2}\right)_{m}\right)_{r e d},
$$

where we consider the reduced structure, and

$$
C_{m}^{\nu}=\overline{D_{m}^{\nu}}
$$

its Zariski closure. We denote by $D(f)$ the open set

$$
D(f)=\operatorname{Spec} R_{f}
$$

where $R$ is the ring $R=\mathbb{C}\left[x_{1}^{(j)}, x_{2}^{(j)}, z^{(j)}\right]_{j \geq 0}$.

Given a jet $\gamma \in X_{m}$, if $x_{i} \circ \gamma \neq 0$ for $i=1,2$, the vector $\nu=\left(\operatorname{ord}_{t}\left(x_{1} \circ \gamma\right)\right.$, $\left.\operatorname{ord}_{t}\left(x_{2} \circ \gamma\right)\right)$ belongs to $\sigma \cap N_{0}$ and $0 \leq \nu_{i} \leq m$. Moreover it is trivial that $\gamma \in C_{m}^{\nu}$. Now we look at $m$-jets with origin at the singular locus.

Lemma 4.2. Given $\gamma \in \pi_{m}^{-1}\left(X_{\text {Sing }}\right)$, there exists $\nu \in \sigma_{\text {Sing }} \cap N_{0}$ with $0 \leq \nu_{i} \leq m$ for $i=1,2$, such that $\gamma \in C_{m}^{\nu}$. Moreover

$$
\pi_{m}^{-1}\left(X_{\text {Sing }}\right)=\bigcup_{\nu \in[0, m]^{2} \cap \sigma_{\text {Sing }} \cap N_{0}} C_{m}^{\nu}
$$

Proof. Given $\gamma \in \pi_{m}^{-1}\left(X_{\text {Sing }}\right)$, suppose first that $x_{i} \circ \gamma \neq 0$ for $i=1,2$. Then $\nu:=\left(\operatorname{ord}_{t}\left(x_{1} \circ \gamma\right)\right.$, ord ${ }_{t}\left(x_{2} \circ\right.$ $\gamma)) \in[0, m]^{2}$ and obviously $\gamma \in D_{m}^{\nu} \subseteq C_{m}^{\nu}$. We have to prove that $\nu \in \sigma_{\text {Sing }} \cap N_{0}$, and this follows by Remark 3.19, since:

(i) If $X_{\text {Sing }}=\{(0,0,0)\}$, then $\gamma(0)=(0,0,0)$ and $\operatorname{ord}_{t}\left(x_{i} \circ \gamma\right)>0$ for $i=1,2$.

(ii) If $X_{\text {Sing }}=Z_{1}$, then $\gamma(0)=\left(0, x_{2}(0), 0\right)$, and $\operatorname{ord}_{t}\left(x_{1} \circ \gamma\right)>0, \operatorname{ord}_{t}\left(x_{2} \circ \gamma\right) \geq 0$.

(iii) If $X_{\text {Sing }}=Z_{1} \cup Z_{2}$, then

$$
\begin{aligned}
& \text { if } \gamma(0) \in Z_{1} \text {, we have } \operatorname{ord}_{t}\left(x_{1} \circ \gamma\right)>0 \text { and } \operatorname{ord}_{t}\left(x_{2} \circ \gamma\right) \geq 0 \\
& \text { if } \gamma(0) \in Z_{2} \text {, we have } \operatorname{ord}_{t}\left(x_{1} \circ \gamma\right) \geq 0 \text { and } \operatorname{ord}_{t}\left(x_{2} \circ \gamma\right)>0
\end{aligned}
$$

To deal with the other cases, notice that

$$
C_{m}^{\nu}=\left\{\gamma \in X_{m} \mid \operatorname{ord}_{t}\left(x_{i} \circ \gamma\right) \geq \nu_{i}, i=1,2\right\}
$$

If $x_{i} \circ \gamma=0$ for $i=1,2$, then $\gamma \in C_{m}^{\nu}$ for any $\nu \in \sigma_{\text {Sing }} \cap N_{0}$ with $0 \leq \nu_{i} \leq m$ for $i=1,2$.

If $x_{1} \circ \gamma=0$ and $x_{2} \circ \gamma \neq 0$, then we denote $\alpha:=\operatorname{ord}_{t}\left(x_{2} \circ \gamma\right)$. We have $0 \leq \alpha \leq m$, and $\gamma \in C_{m}^{\nu}$ for any $\nu \in \sigma_{\text {Sing }} \cap N_{0}$, with $0 \leq \nu_{i} \leq m$ for $i=1,2$, and $\nu_{2} \leq \alpha$.

The left case $x_{1} \circ \gamma \neq 0$ and $x_{2} \circ \gamma=0$ is analogous to the last one.

We prove the other inclusion. If $\gamma \in X_{m} \backslash \pi_{m}^{-1}\left(X_{\text {Sing }}\right)$, then $\gamma(0) \notin X_{\text {Sing }}$. Again distinguishing cases depending on the singular locus, we can prove that $\nu=\left(\operatorname{ord}_{t}\left(x_{1} \circ \gamma\right), \operatorname{ord}_{t}\left(x_{2} \circ \gamma\right)\right) \notin \sigma_{\text {Sing }}$. 
4.1. Description of the sets $C_{m}^{\nu}$. The sets $C_{m}^{\nu}$ are the candidates to be irreducible components of $\pi_{m}^{-1}\left(X_{\text {Sing }}\right)$. We proceed to study these sets. Notice that, by definition, it follows that

$$
C_{m}^{\nu} \subset V\left(x_{i}^{(0)}, \ldots, x_{i}^{\left(\nu_{i}-1\right)}, i=1,2\right) .
$$

Definition 4.3. For $\nu \in \sigma \cap N_{0}$ and $m \in \mathbb{Z}_{>0}$ we define the ideals

$$
\begin{gathered}
I^{\nu}=\left(x_{i}^{(0)}, \ldots, x_{i}^{\left(\nu_{i}-1\right)}\right)_{i=1,2}, \\
J_{m}^{\nu}=\operatorname{Rad}\left(\left(F^{(i)} \bmod I^{\nu}\right)_{0 \leq i \leq m}\right) .
\end{gathered}
$$

Moreover we define the integers $j(m, \nu) \in\{0, \ldots, i(\nu)-1\}$ and $j^{\prime}(m, \nu) \in\{0, \ldots, j(m, \nu)\}$ by the inequalities

$$
\begin{gathered}
l_{j}(\nu) \leq m<l_{j+1}(\nu), \\
l_{j}(\nu)+e_{j} \leq m<l_{j+1}(\nu)+e_{j+1},
\end{gathered}
$$

respectively.

Then we have that

$$
D_{m}^{\nu}=V\left(I^{\nu}, J_{m}^{\nu}\right) \cap D\left(x_{1}^{\left(\nu_{1}\right)}\right) \cap D\left(x_{2}^{\left(\nu_{2}\right)}\right),
$$

where the fact of taking the radical in the definition of $J_{m}^{\nu}$ corresponds to taking the reduced structure in the definition of $D_{m}^{\nu}$. We have to study the polynomials $F^{(j)} \bmod I^{\nu}$ for $0 \leq j \leq m$. Thanks to the identification in (3), these polynomials have to be seen as the defining equations of $D_{m}^{\nu}$.

Example 4.4. Let $X$ be the quasi-ordinary surface defined by the polynomial $f=z^{3}-x_{1}^{3} x_{2}^{2}$. The singular locus of $X$ is reducible

$$
X_{\text {Sing }}=\left\{x_{1}=z=0\right\} \cup\left\{x_{2}=z=0\right\} .
$$

We described in Example 2.1 the equations of the 3-jets, and if we look at 3-jets with origin in the singular locus, then we have to add the condition either $x_{1}^{(0)}=z^{(0)}=0$ or $x_{2}^{(0)}=z^{(0)}=0$. This is equivalent to consider the equations modulo the ideal $I^{\nu}$. Then

$$
\begin{aligned}
\left(\pi_{1}^{-1}\left(X_{\text {Sing }}\right)\right)_{\text {red }} & =\pi_{1}^{-1}\left(\left\{x_{1}=z=0\right\}\right) \cup \pi_{1}^{-1}\left(\left\{x_{2}=z=0\right\}\right) \\
& =V\left(x_{1}^{(0)}, z^{(0)}\right) \cup V\left(x_{2}^{(0)}, z^{(0)}\right)=C_{1}^{(1,0)} \cup C_{1}^{(0,1)} \subset \mathbb{A}_{1}^{3} . \\
\left(\pi_{2}^{-1}\left(X_{\text {Sing }}\right)\right)_{\text {red }} & =\pi_{2}^{-1}\left(\left\{x_{1}=z=0\right\}\right) \cup \pi_{2}^{-1}\left(\left\{x_{2}=z=0\right\}\right) \\
& =V\left(x_{1}^{(0)}, z^{(0)}\right) \cup\left(V\left(x_{2}^{(0)}, z^{(0)}, x_{1}^{(0)^{3}} x_{2}^{(1)^{2}}\right)\right)_{\text {red }}= \\
& =V\left(x_{1}^{(0)}, z^{(0)}\right) \cup V\left(x_{1}^{(0)}, x_{2}^{(0)}, z^{(0)}\right) \cup V\left(x_{2}^{(0)}, x_{2}^{(1)}, z^{(0)}\right)= \\
& =V\left(x_{1}^{(0)}, z^{(0)}\right) \cup V\left(x_{2}^{(0)}, x_{2}^{(1)}, z^{(0)}\right)=C_{2}^{(1,0)} \cup C_{2}^{(0,2)} \subset \mathbb{A}_{2}^{3},
\end{aligned}
$$

since $V\left(x_{1}^{(0)}, x_{2}^{(0)}, z^{(0)}\right) \subset V\left(x_{1}^{(0)}, z^{(0)}\right)$. And

$$
\left(\pi_{3}^{-1}\left(X_{\text {Sing }}\right)\right)_{\text {red }}=V\left(x_{1}^{(0)}, z^{(0)}, z^{(1)^{3}}-x_{1}^{(1)^{3}} x_{2}^{(0)^{2}}\right) \cup V\left(x_{2}^{(0)}, x_{2}^{(1)}, z^{(0)}, z^{(1)}\right)=C_{3}^{(1,0)} \cup C_{3}^{(0,2)} \subset \mathbb{A}_{3}^{3} .
$$

In this example we see how the components are defined by hyperplane coordinates for $m<3$, and at level $m=3$ the equation $f$ starts playing a role. When there are more than one approximated root, the approximated roots affect the geometry of $C_{m}^{\nu}$ one after the other as $m$ grows. This will be explained in Proposition 4.13, We illustrate this with another example.

Example 4.5. Consider the quasi-ordinary surface $f=\left(z^{2}-x_{1}^{3}\right)^{3}-x_{1}^{10} x_{2}^{4}$. The generators of the semigroup are $\gamma_{1}=\left(\frac{3}{2}, 0\right)$ and $\gamma_{2}=\left(\frac{10}{3}, \frac{4}{3}\right)$, and the singular locus is $X_{\text {Sing }}=\left\{x_{1}=z=0\right\} \cup\left\{x_{2}=z^{2}-x_{1}^{3}=0\right\}$. If we lift the component of the singular locus $Z_{2}=\left\{x_{2}=f_{1}=0\right\}$ at level 3 , we have that $\left(\pi_{3}^{-1}\left(Z_{2}\right)\right)_{\text {red }}=$ 


$$
\begin{aligned}
V\left(x_{2}^{(0)}, F_{1}^{(0)}, F_{2}^{(1)}, F_{2}^{(2)}, F_{2}^{(3)}\right), \text { where } & F_{1}^{(0)}=z^{(0)^{2}}-x_{1}^{(0)^{3}} \text {, and } \\
F_{2}^{(1)} & \equiv 3 F_{1}^{(0)^{2}} F_{1}^{(1)} \bmod \left(x_{2}^{(0)}, z^{(0)}\right) \\
& \equiv 0 \bmod \left(x_{2}^{(0)}, z^{(0)}, F_{1}^{(0)}\right) \\
F_{2}^{(2)} & \equiv 3 F_{1}^{(0)^{2}} F_{1}^{(2)}+6 F_{1}^{(0)} F_{1}^{(1)^{2}} \bmod \left(x_{2}^{(0)}, z^{(0)}\right) \\
& \equiv 0 \bmod \left(x_{2}^{(0)}, z^{(0)}, F_{1}^{(0)}\right) \\
F_{2}^{(3)} & \equiv F_{1}^{(1)^{3}} \bmod \left(x_{2}^{(0)}, z^{(0)}, F_{1}^{(0)}\right),
\end{aligned}
$$

and then $\left(\pi_{3}^{-1}\left(Z_{2}\right)\right)_{\text {red }}=V\left(x_{2}^{(0)}, F_{1}^{(0)}, F_{1}^{(1)}\right)$. Notice that it is not a component of $\left(\pi_{3}^{-1}\left(X_{\text {Sing }}\right)\right)_{\text {red }}$, since it is not irreducible. Indeed, it decomposes as

$$
\left(\pi_{3}^{-1}\left(Z_{2}\right)\right)_{\text {red }}=V\left(x_{1}^{(0)}, x_{2}^{(0)}, z^{(0)}\right) \cup \overline{V\left(x_{2}^{(0)}, F_{1}^{(0)}, F_{1}^{(1)}\right) \cap D\left(x_{1}^{(0)}\right)} .
$$

We have seen in this example how, to give a minimal set of generators of $J_{m}^{\nu}$, we need to study the polynomials $F^{(l)} \bmod J_{l-1}^{\nu}$. Therefore we introduce the following definition.

Definition 4.6. For $\nu \in \sigma_{\text {Sing }} \cap N_{0}$ and $0 \leq l \leq m$, we denote by

$$
F_{\nu}^{(l)}:=F^{(l)} \bmod \left(I^{\nu}, J_{l-1}^{\nu}\right)
$$

and for the approximated roots the notation is $F_{j, \nu}^{(l)}$.

And now we obviously have that

$$
J_{m}^{\nu}=\operatorname{Rad}\left(F_{\nu}^{(0)}, \ldots, F_{\nu}^{(m)}\right) .
$$

Regarding the claim in Remark 2.2, once we consider $F_{\nu}^{(l)}$, it is not true anymore that the polynomials are non-zero. But, whenever $F_{\nu}^{(l)}$ is non-zero, then it is quasi-homogeneous of degree $l$.

In general, the first approximated root which appears is not necessarily the first one, and the process does not finish with the last one. To control, for a given $\nu$, all this behaviour, we defined the integers $i(\nu), c(\nu)$ and $m(\nu)$ in Definition 3.20. Indeed, given $m \in \mathbb{Z}_{>0}$ and $\nu \in \sigma_{S i n g} \cap N_{0}$ such that $l_{c(\nu)}(\nu) \leq m$, the approximated roots which will influence the defining ideal of $D_{m}^{\nu}$, are

$$
f_{c(\nu)}, \ldots, f_{j(m, \nu)},
$$

where remember the convention $f_{0}=z$. Moreover, the moment when $f_{i}$ begins to influence the defining equations of $C_{m}^{\nu}$ (or in other words, the generators of $J_{m}^{\nu}$ ) for the first time is exactly at $m=l_{i}(\nu)$. This is the content of Corollary 4.15. The meaning of the integer $i(\nu)$ is that, at $m=l_{i(\nu)}(\nu), \nu$ does no longer give rise to an irreducible component (see Lemma 4.8). The integer $j(m, \nu)$ will be useful to describe the component $C_{m}^{\nu}$ (see Proposition 4.13), while $j^{\prime}(m, \nu)$ will be crucial when studying the inclusion $C_{m}^{\nu^{\prime}} \subseteq C_{m}^{\nu}$ (see Proposition 4.21).

Example 4.7. We revisit Example 4.4. If we lift the component $C_{3}^{(0,2)}$ to level 4 , we have

$$
\pi_{4,3}^{-1}\left(C_{3}^{(0,2)}\right)=V\left(x_{2}^{(0)}, x_{2}^{(1)}, z^{(0)}, z^{(1)}, F^{(4)}\right)
$$

where $F^{(4)} \equiv x_{1}^{(0)^{3}} x_{2}^{(2)^{2}} \bmod \left(x_{2}^{(0)}, x_{2}^{(1)}, z^{(0)}, z^{(1)}\right)$. Therefore

$$
\pi_{4,3}^{-1}\left(C_{3}^{(0,2)}\right)=C_{4}^{(1,2)} \cup C_{4}^{(0,3)} .
$$

Then at level $m=4$, the vector $(0,2)$ does not give rise to an irreducible component any longer. The reason is that $(0,2) \notin N_{1}$ and $4=l_{1}(0,2)$.

This is the case in general as we claim in the next Lemma, whose proof is left to Section 5

Lemma 4.8. For $m \in \mathbb{Z}_{>0}$ and $\nu \in[0, m]^{2} \cap \sigma_{\text {Sing }} \cap N_{0}$, we have

$$
D_{m}^{\nu}=\emptyset \text { if and only if } m \geq l_{i(\nu)}(\nu) \text {. }
$$


As a consequence of this Lemma, we are going to prove an improvement of Lemma 4.2, namely, for $m \in \mathbb{Z}_{>0}$, to cover $\pi_{m}^{-1}\left(X_{\text {Sing }}\right)$ it is enough to consider $\nu \in[0, m]^{2} \cap \sigma_{\text {Sing }} \cap N_{0}$ with $m<l_{i(\nu)}(\nu)$.

Notice that, if $l_{m(\nu)}(\nu) \leq m$, then $l_{1}(\nu) \leq l_{m(\nu)}(\nu) \leq m$, and by the previous Lemma, we have to ask $\nu \in N_{1}$ whenever $l_{m(\nu)} \leq m$.

Definition 4.9. Given $m \in \mathbb{Z}_{>0}$ we define the sets:

$$
\begin{aligned}
& H_{m}=\left\{\nu \in[0, m]^{2} \cap \sigma_{\text {Sing }} \cap N_{0} \mid l_{m(\nu)}(\nu) \geq m+1\right\}, \\
& L_{m}=\left\{\nu \in[0, m]^{2} \cap \sigma_{\text {Sing }} \cap N_{1} \mid l_{m(\nu)}(\nu) \leq m<l_{i(\nu)}(\nu)\right\} .
\end{aligned}
$$

It will be necessary later to subdivide the set $L_{m}$ as

$$
\begin{aligned}
& L_{m}^{=}=\left\{\nu \in L_{m} \mid l_{m(\nu)}(\nu) \leq m<\min \left\{l_{m(\nu)}(\nu)+e_{m(\nu)}, l_{i(\nu)}(\nu)\right\}\right\}, \\
& L_{m}^{<}=\left\{\nu \in L_{m} \mid l_{m(\nu)}(\nu)+e_{m(\nu)} \leq m<l_{i(\nu)}(\nu)\right\} .
\end{aligned}
$$

If we come back to Example 4.4, we have that

$$
\begin{array}{ll}
\left(\pi_{1}^{-1}\left(X_{\text {Sing }}\right)\right)_{\text {red }}=C_{1}^{(1,0)} \cup C_{1}^{(0,1)} & \text { with }(1,0),(0,1) \in H_{1}, \\
\left(\pi_{2}^{-1}\left(X_{\text {Sing }}\right)\right)_{\text {red }}=C_{2}^{(1,0)} \cup C_{2}^{(0,2)} & \text { with }(1,0),(0,2) \in H_{2}, \\
\left(\pi_{3}^{-1}\left(X_{\text {Sing }}\right)\right)_{\text {red }}=C_{3}^{(1,0)} \cup C_{3}^{(0,2)} & \text { with }(1,0) \in L_{3}^{=} \text {and }(0,2) \in H_{3} .
\end{array}
$$

Lemma 4.10. For $m \in \mathbb{Z}_{>0}$, we have that $H_{m} \cup L_{m} \neq \emptyset$, and

$$
\pi_{m}^{-1}\left(X_{\text {Sing }}\right)=\bigcup_{\nu \in H_{m} \cup L_{m}} C_{m}^{\nu}
$$

Proof. The first claim follows because $(m, m) \in H_{m}$ for any $m \in \mathbb{Z}_{>0}$. Indeed, since $l_{m(\nu)}(\nu) \geq l_{1}(\nu)=$ $n_{1} e_{1}\left\langle\nu, \gamma_{1}\right\rangle=e_{1} m\left(a_{1}+b_{1}\right)>m$, where the last inequality holds because $a_{1}+b_{1}>1$, since the branch is normalized. By Lemma 4.2

$$
\bigcup_{\nu \in H_{m} \cup L_{m}} C_{m}^{\nu} \subseteq \bigcup_{\nu \in[0, m]^{2} \cap \sigma_{\text {Sing }} \cap N_{0}} C_{m}^{\nu}=\pi_{m}^{-1}\left(X_{\text {Sing }}\right) .
$$

We prove the other inclusion. Notice that $\nu \notin H_{m} \cup L_{m}$ is equivalent to $l_{i(\nu)}(\nu) \leq m$, because $m(\nu) \leq i(\nu)$. For any $\gamma \in \pi_{m}^{-1}\left(X_{\text {Sing }}\right)$,

- if $x_{i} \circ \gamma \neq 0$ for $i=1,2$, then $\nu:=\left(\operatorname{ord}_{t}\left(x_{1} \circ \gamma\right), \operatorname{ord}_{t}\left(x_{2} \circ \gamma\right)\right) \in[0, m]^{2}$ and $\gamma \in D_{m}^{\nu}$. Hence, by Lemma 4.8 we have that $m<l_{i(\nu)}(\nu)$ and therefore $\nu \in H_{m} \cup L_{m}$.

Otherwise,

- if $x_{i} \circ \gamma=0$ for $i=1,2$ we saw in the proof of Lemma 4.2 that $\gamma \in C_{m}^{\nu}$ for any $\nu \in[0, m]^{2} \cap \sigma_{\text {Sing }} \cap N_{0}$. Therefore, since $H_{m} \cap L_{m} \neq \emptyset$, there exists $\nu$ with $\gamma \in C_{m}^{\nu}$.

- if $x_{1} \circ \gamma=0$ and $x_{2} \circ \gamma \neq 0$, then by the proof of Lemma 4.2, we have that $\gamma \in C_{m}^{(m, \alpha)}$, where $\alpha=\operatorname{ord}_{t}\left(x_{2} \circ \gamma\right)$. We have to prove that $\nu:=(m, \alpha) \in H_{m}$, and this follows since $l_{m(\nu)}(\nu) \geq l_{1}(\nu)=$ $e_{1}\left(a_{1} m+b_{1} \alpha\right)>m$, again using that the branch is normalized.

- if $x_{1} \circ \gamma \neq 0$ and $x_{2} \circ \gamma=0$, then by the proof of Lemma 4.2 $\gamma \in C_{m}^{\nu}$ for any $\nu$ with $\nu_{1} \leq \alpha$ and $\nu_{2} \leq m$, where $\alpha=\operatorname{ord}_{t}\left(x_{1} \circ \gamma\right)$. Hence we only have to prove that $([0, \alpha] \times[0, m]) \cap\left(H_{m} \cup L_{m}\right) \neq \emptyset$. If $b_{1} \geq 1$ then $\nu:=(\alpha, m) \in H_{m}$. Indeed, if $b_{1}>1$ clearly $l_{m(\nu)}(\nu) \geq l_{1}(\nu)=e_{1}\left(a_{1} \alpha+b_{1} m\right)>m$. The same works if $b_{1}=1$ and $g>1$, because then $e_{1}>1$. If $b_{1}=1$ and $g=1$, then by Theorem 3.16 we deduce that $\rho_{2} \nsubseteq \sigma_{\text {Sing }}$. Then $\alpha>0$ and it follows that $\nu \in H_{m}$. The case left is $b_{1}=0$. In this case, if we set $\nu:=(\alpha, m)$, we have $m(\nu)=g_{1}$ and $i(\nu) \geq g_{1}+1$. Then $l_{i(\nu)}(\nu) \geq l_{g_{1}+1}(\nu)=e_{g_{1}+1}\left(\alpha n_{g_{1}+1} \gamma_{g_{1}+1}^{(1)}+m n_{g_{1}+1} \gamma_{g_{1}+1}^{(2)}\right)>m$, where we are using that if $\alpha=0$ then $\rho_{2} \subseteq \sigma_{\text {Sing }}$ and $n_{g_{1}+1} \gamma_{g_{1}+1}^{(2)}>1$. If $\alpha \geq n_{1}$, the same argument shows that $\left(n_{1}, m\right) \in L_{m}$. Otherwise $(\alpha, m) \in L_{m}$, since $\gamma \in X_{m}$ and $\gamma_{1}=\left(\frac{a_{1}}{n_{1}}, 0\right)$, therefore $a_{1} \operatorname{ord}_{t}\left(x_{1} \circ \gamma\right)=n_{1} \operatorname{ord}_{t}(z \circ \gamma)$, which implies that $\alpha \frac{a_{1}}{n_{1}} \in \mathbb{Z}$ or in other words, $(\alpha, m) \in N_{1}$. 
Given $\nu \in \sigma_{\text {Sing }} \cap N_{0}$, it gives rise to a candidate of irreducible component at level $m$, $C_{m}^{\nu}$, for

$$
0=l_{c(\nu)}(\nu) \leq m<l_{i(\nu)}(\nu) .
$$

Remark 4.11. (i) If $\nu \in H_{m}$, then $c(\nu)=0$, since otherwise $m(\nu)=c(\nu)$ and $l_{m(\nu)}(\nu)=0<m+1$, or in other words, if $g_{1}>0$ then $H_{m} \cap \rho_{2}=\emptyset$.

(ii) It is clear that $j(m, \nu)=0$ if and only if $\nu \in H_{m}$ and $j(m, \nu) \geq 1$ if and only if $\nu \in L_{m}$.

For $\nu \in H_{m}$ the sets $C_{m}^{\nu}$ are very easy to describe, as we see in the next Proposition.

Proposition 4.12. For $m \in \mathbb{Z}_{>0}$ and $\nu \in H_{m}$ we have that

$$
J_{m}^{\nu}=\left(z^{(0)}, \ldots, z^{([m / n])}\right)
$$

and hence the set $C_{m}^{\nu}$ is defined by hyperplane coordinates in $\mathbb{A}_{m}^{3}$ as

$$
C_{m}^{\nu}=V\left(x_{i}^{(0)}, \ldots, x_{i}^{\left(\nu_{i}-1\right)}, i=1,2 ; z^{(0)}, \ldots, z^{\left(\left[\frac{m}{n}\right]\right)}\right) .
$$

Proof. The proof is by induction on $m$. For $m=1$ we have

$$
H_{1}= \begin{cases}\{(1,1)\} & \text { if } \gamma_{1}=\left(\frac{1}{n}, \frac{1}{n}\right) \text { and } g=1 \\ \{(1,0),(1,1)\} & \text { if } g_{1}>0 \text { (recall that the branch is normalized) } \\ \{(1,0),(1,1),(0,1)\} & \text { otherwise }\end{cases}
$$

and the claim follows, since

$$
C_{1}^{(1,0)}=V\left(x_{1}^{(0)}, z^{(0)}\right), C_{1}^{(1,1)}=V\left(x_{1}^{(0)}, x_{2}^{(0)}, z^{(0)}\right) \text { and } C_{1}^{(0,1)}=V\left(x_{2}^{(0)}, z^{(0)}\right) \text { if } g_{1}=0 .
$$

Suppose the claim is true for $m$ and we will prove it for $m+1$. Given $\nu \in H_{m+1}$, since $\nu \in H_{m}$, by induction hypothesis we have that

We claim that

$$
J_{m+1}^{\nu}=\left(z^{(0)}, \ldots, z^{([m / n])}, F_{\nu}^{(m+1)}\right)
$$

$$
F_{\nu}^{(m+1)}=\left\{\begin{array}{cl}
0 & \text { if } m+1 \not \equiv 0 \bmod n \\
z^{\left(\frac{m+1}{n}\right)^{n}} & \text { otherwise }
\end{array}\right.
$$

which proves the result, since

$$
\left[\frac{m+1}{n}\right]= \begin{cases}{\left[\frac{m}{n}\right]} & \text { if } m+1 \not \equiv 0 \bmod n \\ \frac{m+1}{n} & \text { otherwise }\end{cases}
$$

If $F_{\nu}^{(m+1)} \neq 0$ then it is a quasi-homogeneous polynomial of degree $m+1$. By the expansion (12) given in Lemma 3.13

$$
f=z^{n}+\sum c_{\underline{\alpha}, \underline{r}} x_{1}^{\alpha_{1}} x_{2}^{\alpha_{2}} z^{r_{1}}
$$

where $\left(\alpha_{1}, \alpha_{2}\right)+r_{1} \gamma_{1}>n_{1} e_{1} \gamma_{1}=n \gamma_{1}$. Then any monomial in $f-z^{n}$ verifies for any $\gamma \in D_{m}^{\nu}$

$$
\begin{aligned}
\operatorname{ord}_{t}\left(c_{\underline{\alpha}, \underline{r}} x_{1}^{\alpha_{1}} x_{2}^{\alpha_{2}} \circ \gamma\right) & =\left\langle\nu,\left(\alpha_{1}, \alpha_{2}\right)\right\rangle+r_{1} \operatorname{ord}_{t}(z \circ \gamma) \\
& \geq\left\langle\nu,\left(\alpha_{1}, \alpha_{2}\right)\right\rangle+r_{1} \frac{m+1}{n} \\
& \geq n\left\langle\nu, \gamma_{1}\right\rangle-r_{1}\left\langle\nu, \gamma_{1}\right\rangle+r_{1} \frac{m+1}{n} \\
& =\left(n-r_{1} \frac{l_{1}(\nu)}{n}+r_{1} \frac{m+1}{n}>\left(n-r_{1}\right) \frac{m+1}{n}+r_{1} \frac{m+1}{n}=m+1\right.
\end{aligned}
$$

because $\nu \in H_{m+1}$ and by induction hypothesis $\operatorname{ord}_{t}(z \circ \gamma)>\left[\frac{m}{n}\right]$ (and therefore $\geq \frac{m+1}{n}$ ). Hence these monomials do not contribute to $F_{\nu}^{(m+1)}$ and the result follows by the quasi-homogeneity of $F_{\nu}^{(m+1)}$.

For $\nu \in L_{m}$ the geometry of $C_{m}^{\nu}$ is much more complicated, the ideal $J_{m}^{\nu}$ is described in Corollary 4.15, In the next Proposition we compare jet schemes of a quasi-ordinary singularity with jet schemes of its approximated roots. For $1 \leq i \leq g, m \in \mathbb{Z}_{>0}$ and $\nu \in H_{m} \cup L_{m}$, we denote by $D_{i, m}^{\nu}$ the set

$$
D_{i, m}^{\nu}=\left\{\gamma \in X_{m}^{(i)} \mid \operatorname{ord}_{t}\left(x_{k} \circ \gamma\right)=\nu_{k}, k=1,2\right\}_{\text {red }}
$$

and we denote $D_{g, m}^{\nu}$ simply by $D_{m}^{\nu}$ (see Definition 3.4 for the definition of $X^{(i)}$ ). 
Proposition 4.13. For $m \in \mathbb{Z}_{>0}$ and $\nu \in H_{m} \cup L_{m}$, we have that

$$
D_{m}^{\nu}=\left(\pi_{m,\left[\frac{m}{e_{j}}\right]}^{a}\right)^{-1}\left(D_{j,\left[\frac{m}{e_{j}}\right]}^{\nu}\right)
$$

where $j=j(m, \nu)$, and for $q>p, \pi_{q, p}^{a}: \mathbb{A}_{q}^{3} \longrightarrow \mathbb{A}_{p}^{3}$ is the projection on the jet schemes of the affine ambient space.

Hence, for $m \in \mathbb{Z}_{>0}$ and $\nu \in L_{m}$ with $j(m, \nu)=j$, the geometry of $C_{m}^{\nu}$ is determined by the geometry of the $j$-th approximated root.

Before proving the Proposition we need the following technical result, whose proof is moved to Section 5 .

Lemma 4.14. For $m \in \mathbb{Z}_{>0}$ and $\nu \in H_{m} \cup L_{m}$, we have that for all $\gamma \in D_{m}^{\nu}$,

$$
\begin{aligned}
& \operatorname{ord}_{t}\left(f_{k} \circ \gamma\right)=\left\langle\nu, \gamma_{k+1}\right\rangle, \quad \text { for } 0 \leq k \leq j(m, \nu)-1 \\
& \operatorname{ord}_{t}\left(f_{k} \circ \gamma\right)>\frac{m}{e_{k}}, \quad \text { for } j(m, \nu) \leq k \leq g
\end{aligned}
$$

Proof of Proposition 4.13. For $\nu \in H_{m}$ we have $j(m, \nu)=0$, and the claim follows by Proposition 4.12. For $\nu \in L_{m}$ it is enough to prove that, if $j(m, \nu)=j$ we have

$$
D_{m}^{\nu}=\left\{\gamma \in \mathbb{A}_{m}^{3} \mid \operatorname{ord}_{t}\left(x_{i} \circ \gamma\right)=\nu_{i}, i=1,2 \text { and } \operatorname{ord}_{t}\left(f_{j} \circ \gamma\right)>\frac{m}{e_{j}}\right\}
$$

By Lemma 4.14 it follows that

$$
D_{m}^{\nu} \subseteq\left\{\gamma \in \mathbb{A}_{m}^{3} \mid \operatorname{ord}_{t}\left(x_{i} \circ \gamma\right)=\nu_{i}, i=1,2 \text { and } \operatorname{ord}_{t}\left(f_{j} \circ \gamma\right)>\frac{m}{e_{j}}\right\}
$$

We prove the other inclusion. Let $\gamma$ be a jet with $\operatorname{ord}_{t}\left(x_{i} \circ \gamma\right)=\nu_{i}$ for $i=1,2$ and $\operatorname{ord}_{t}\left(f_{j} \circ \gamma\right)>\frac{m}{e_{j}}$. We want to prove that it is indeed an $m$-jet in $X$, or in other words, that $\operatorname{ord}_{t}(f \circ \gamma) \geq m+1$. Notice that if $j=g$ there is nothing to prove. Then $j<g$, and first we will prove that

$$
\operatorname{ord}_{t}\left(f_{j+1} \circ \gamma\right)>\frac{m}{e_{j+1}} \text {. }
$$

Indeed, consider $f_{j}$ quasi-ordinary surface with $j$ characteristic exponents. If $\bar{m}:=\left[\frac{m}{e_{j}}\right]$ and $\bar{\gamma}=\pi_{m, \bar{m}}(\gamma)$, then we have that $\bar{\gamma} \in D_{j, \bar{m}}^{\nu}$. Moreover $n_{j}\left\langle\nu, \gamma_{j}\right\rangle \leq \bar{m}$, and then, by Lemma 4.14 applied to $f_{j}$, we have

$$
\operatorname{ord}_{t}\left(f_{k} \circ \bar{\gamma}\right)=\left\langle\nu, \gamma_{k+1}\right\rangle, \text { for } 0 \leq k \leq j-1 \text {. }
$$

Since $\left\langle\nu, \gamma_{k+1}\right\rangle \leq\left\langle\nu, \gamma_{j}\right\rangle<\bar{m}<m$, we deduce that $\operatorname{ord}_{t}\left(f_{k} \circ \gamma\right)=\operatorname{ord}_{t}\left(f_{k} \circ \bar{\gamma}\right)$.

Now we consider $f_{j+1}$. By Lemma 3.12 we have

$$
f_{j+1}=f_{j}^{n_{j+1}}-c_{j+1} x_{1}^{\alpha_{1}^{(j+1)}} x_{2}^{\alpha_{2}^{(j+1)}} z^{r_{1}^{(j+1)}} \cdots f_{j-1}^{r_{j}^{(j+1)}}+\sum c_{\underline{\alpha}, \underline{\underline{r}}} x_{1}^{\alpha_{1}} x_{2}^{\alpha_{2}} z^{r_{1}} \cdots f_{j}^{r_{j+1}}
$$

and using that $\operatorname{ord}_{t}\left(f_{k} \circ \gamma\right)=\left\langle\nu, \gamma_{k+1}\right\rangle$ for $0 \leq k \leq j-1$ we have

$$
\begin{aligned}
\operatorname{ord}_{t}\left(f_{j}^{n_{j+1}} \circ \gamma\right) & =n_{j+1} \operatorname{ord}_{t}\left(f_{j} \circ \gamma\right)>\frac{m}{e_{j+1}} \\
\operatorname{ord}_{t}\left(\left(x_{1}^{\alpha_{1}^{(j+1)}} x_{2}^{\alpha_{2}^{(j+1)}} z^{r_{1}^{(j+1)}} \cdots f_{j-1}^{r_{j}^{(j+1)}}\right) \circ \gamma\right) & =\left\langle\nu,\left(\alpha_{1}^{(j+1)}, \alpha_{2}^{(j+1)}\right)+r_{1}^{(j+1)} \gamma_{1}+\cdots+r_{j}^{(j+1)} \gamma_{j}\right\rangle \\
& =n_{j+1}\left\langle\nu, \gamma_{j+1}\right\rangle>\frac{m}{e_{j+1}} \\
\operatorname{ord}_{t}\left(\left(c_{\underline{\alpha}, \underline{r}} x_{1}^{\alpha_{1}} x_{2}^{\alpha_{2}} z^{r_{1}} \cdots f_{j}^{r_{j+1}}\right) \circ \gamma\right) & =\left\langle\nu,\left(\alpha_{1}, \alpha_{2}\right)+r_{1} \gamma_{1}+\cdots+r_{j} \gamma_{j}\right\rangle+r_{j+1} \operatorname{ord}_{t}\left(f_{j} \circ \gamma\right)
\end{aligned}
$$

If $\operatorname{ord}_{t}\left(f_{j+1} \circ \gamma\right) \leq \frac{m}{e_{j+1}}$, then there must exist $c_{\underline{\alpha}, \underline{r}} \neq 0$ such that

$$
\operatorname{ord}_{t}\left(f_{j+1} \circ \gamma\right)=\left\langle\nu,\left(\alpha_{1}, \alpha_{2}\right)+r_{1} \gamma_{1}+\cdots+r_{j} \gamma_{j}\right\rangle+r_{j+1} \operatorname{ord}_{t}\left(f_{j} \circ \gamma\right) \leq \frac{m}{e_{j+1}}
$$


and we get the following inequalities

$$
\begin{aligned}
\left(n_{j+1}-r_{j+1}\right)\left\langle\nu, \gamma_{j+1}\right\rangle+r_{j+1} \frac{m}{e_{j}} & \leq\left\langle\nu,\left(\alpha_{1}, \alpha_{2}\right)+r_{1} \gamma_{1}+\cdots+r_{j} \gamma_{j}\right\rangle+r_{j+1} \frac{m}{e_{j}} \\
& <\left\langle\nu,\left(\alpha_{1}, \alpha_{2}\right)+r_{1} \gamma_{1}+\cdots+r_{j} \gamma_{j}\right\rangle+r_{j+1} \operatorname{ord}_{t}\left(f_{j} \circ \gamma\right) \\
& \leq \frac{m}{e_{j+1}}
\end{aligned}
$$

Hence $\left(n_{j+1}-r_{j+1}\right)\left\langle\nu, \gamma_{j+1}\right\rangle+r_{j+1} \frac{m}{e_{j}}<\frac{m}{e_{j+1}}=n_{j+1} \frac{m}{e_{j}}$, and then $\left(n_{j+1}-r_{j+1}\right)\left\langle\nu, \gamma_{j+1}\right\rangle<\left(n_{j+1}-r_{j+1}\right) \frac{m}{e_{j}}$. Since $r_{j+1}<n_{j+1}$, we have

$$
\left\langle\nu, \gamma_{j+1}\right\rangle<\frac{m}{e_{j}}
$$

which is in contradiction with $j(m, \nu)=j$. Therefore we have just proved that $\operatorname{ord}_{t}\left(f_{j+1} \circ \gamma\right)>\frac{m}{e_{j+1}}$.

To finish, by Lemma 3.13 we have

$$
f=f_{j+1}^{e_{j+1}}+\sum_{\left(i_{1}, i_{2}\right)+k \gamma_{1}>n_{j+1} e_{j+1} \gamma_{j+1}} c_{i_{1} i_{2} k} x_{1}^{i_{1}} x_{2}^{i_{2}} z^{k},
$$

and

$$
\begin{gathered}
\operatorname{ord}_{t}\left(f_{j+1}^{e_{j+1}} \circ \gamma\right)=e_{j+1} \operatorname{ord}_{t}\left(f_{j+1} \circ \gamma\right)>m \\
\operatorname{ord}_{t}\left(\left(c_{i_{1} i_{2} k} x_{1}^{i_{1}} x_{2}^{i_{2}} z^{k}\right) \circ \gamma\right) \geq n_{j+1} e_{j+1}\left\langle\nu, \gamma_{j+1}\right\rangle=l_{j+1}(\nu)>m
\end{gathered}
$$

Hence $\operatorname{ord}_{t}(f \circ \gamma)>m$ as we wanted to prove.

As a consequence of Proposition 4.13, we have the following algebraic counterpart, where we explain how the equations of the approximated roots appear as generators of $J_{m}^{\nu}$, and therefore a minimal presentation of the ideal $J_{m}^{\nu}$ is given.

Corollary 4.15. Given $m \in \mathbb{Z}_{>0}$ and $\nu \in H_{m} \cup L_{m}$, for $l_{i}(\nu) \leq l<l_{i+1}(\nu)$ (resp. $\left.l_{i}(\nu) \leq l \leq m\right)$ if $c(\nu) \leq i<j(m, \nu)$ (resp. $i=j(m, \nu))$, we have that

$$
F_{\nu}^{(l)}=\left\{\begin{array}{cl}
F_{i, \nu}^{\left(\frac{l}{e_{i}}\right)^{e_{i}}} & \text { if } l \equiv 0 \bmod e_{i} \\
0 & \text { otherwise }
\end{array}\right.
$$

Hence the ideal $J_{m}^{\nu}$ is generated by the polynomials

$$
J_{m}^{\nu}=\left(F_{i, \nu}^{\left(\frac{l_{i}(\nu)}{e_{i}}+k_{i}\right)}\right)_{i}
$$

for $c(\nu) \leq i \leq j(m, \nu)$ such that $l_{i}(\nu)<l_{i+1}(\nu)$, and $0 \leq k_{i}<\frac{l_{i+1}(\nu)-l_{i}(\nu)}{e_{i}}$ if $i<j(m, \nu)$ and $0 \leq k_{j(m, \nu)} \leq$ $\left[\frac{m-l_{j(m, \nu)}(\nu)}{e_{j(m, \nu)}}\right]$. Moreover, we have

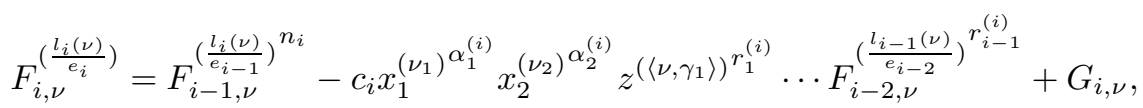

where $G_{i, \nu}$ is the polynomial

$$
G_{i, \nu}=\sum c_{\underline{\alpha}, \underline{r}} x_{1}^{\left(\nu_{1}\right)^{\alpha_{1}}} x_{2}^{\left(\nu_{2}\right)^{\alpha_{2}}} z^{\left(\left\langle\nu, \gamma_{1}\right\rangle\right)^{r_{1}}} \cdots F_{i-2, \nu}^{\left(\frac{l_{i-1}(\nu)}{e_{i-2}}\right)^{r_{i-1}}} F_{i-1, \nu}^{\left(\frac{l_{i}(\nu)}{e_{i-1}}\right)^{r_{i}}}
$$

with $c_{\underline{\alpha}, \underline{\underline{r}}}$ are the coefficients appearing in the expansion given in Lemma 3.12 , and such that

(i) $c_{\underline{\alpha}, \underline{r}} \neq 0$

(ii) $\left\langle\nu,\left(\alpha_{1}, \alpha_{2}\right)+r_{1} \gamma_{1}+\cdots+r_{i} \gamma_{i}\right\rangle=n_{i}\left\langle\nu, \gamma_{i}\right\rangle$

Notice that condition (ii) does not hold when $\nu \notin \rho_{1} \cup \rho_{2}$, and hence $G_{i, \nu}=0$ in these cases.

Proof. It is a consequence of Proposition 4.13, applied to any $l_{i}(\nu) \leq l<l_{i+1}(\nu)$, and using the trivial observation that $J_{m^{\prime}}^{\nu} \subseteq J_{m}^{\nu}$ for any $m^{\prime}<m$. Indeed, for any $l_{i}(\nu) \leq l<l_{i+1}(\nu)$ we study the polynomials 
$F_{\nu}^{\left(l_{i}(\nu)\right)}, \ldots, F_{\nu}^{(l)}$ (note that we need $\left.l_{i}(\nu)<l_{i+1}(\nu)\right)$. We have that $j(l, \nu)=i$, and, by Proposition 4.13, $D_{l}^{\nu}=\left(\pi_{l,\left[\frac{l}{e_{i}}\right]}^{a}\right)^{-1}\left(D_{i,\left[\frac{l}{e_{i}}\right]}^{\nu}\right)$, or in other words

$$
D_{l}^{\nu}=\left\{\gamma \in \mathbb{A}_{l}^{3} \mid \operatorname{ord}_{t}\left(x_{k} \circ \gamma\right)=\nu_{k}, k=1,2, \operatorname{ord}_{t}\left(f_{i} \circ \gamma\right)>\left[\frac{l}{e_{i}}\right]\right\}
$$

Then the ideal $J_{l}^{\nu}$ only depends on $f_{i}$ (and hence on its approximated roots). Moreover, by Lemma 4.14, we deduce that for $0 \leq k<i$

$$
F_{k, \nu}^{\left(r_{k}\right)} \in J_{l}^{\nu}, \text { for } 0 \leq r_{k}<\left\langle\nu, \gamma_{k+1}\right\rangle
$$

By the expansion (12) in Lemma 3.13

$$
f=f_{i}^{e_{i}}+\sum c_{\underline{\alpha}, \underline{r}} x_{1}^{\alpha_{1}} x_{2}^{\alpha_{2}} z^{r_{1}} \cdots f_{i}^{r_{i+1}}
$$

where $\left(\alpha_{1}, \alpha_{2}\right)+r_{1} \gamma_{1}+\cdots+r_{i+1} \gamma_{i+1}>n_{i+1} e_{i+1} \gamma_{i+1}$.

The part $f_{i}^{e_{i}}$ contributes to $F^{(l)}$ with $F_{i, \nu}^{\left(\frac{l}{e_{i}}\right)^{e_{i}}}$ and only when $l$ is divisible by $e_{i}$. While for the monomials $x_{1}^{\alpha_{1}} x_{2}^{\alpha_{2}} z^{r_{1}} \cdots f_{i}^{r_{i+1}}$ the contribution is given by

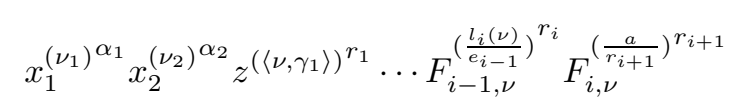

with $\left\langle\nu,\left(\alpha_{1}, \alpha_{2}\right)+r_{1} \gamma_{1}+\cdots+r_{i} \gamma_{i}\right\rangle+a=l$, and only when $a$ is divisible by $r_{i+1}$. Set $a=b r_{i+1}$, we claim that $F_{i, \nu}^{(b)}$ belongs to $J_{l}^{\nu}$ and hence the monomial does not contribute to $F^{(l)}$. Indeed, at level $l^{\prime}=b e_{i}$ it appears as $F_{i, \nu}^{(b)^{e_{i}}}$, and we only need to prove that $l^{\prime}<l$. Since

$$
\begin{aligned}
l & =\left\langle\nu,\left(\alpha_{1}, \alpha_{2}\right)+r_{1} \gamma_{1}+\cdots+r_{i} \gamma_{i}\right\rangle+r_{i+1} b \\
& =\left\langle\nu,\left(\alpha_{1}, \alpha_{2}\right)+r_{1} \gamma_{1}+\cdots+r_{i} \gamma_{i}\right\rangle+r_{i+1} \frac{l^{\prime}}{e_{i}}
\end{aligned}
$$

we have

$$
\begin{aligned}
l^{\prime} & =\frac{e_{i}}{r_{i+1}}\left(l-\left\langle\nu,\left(\alpha_{1}, \alpha_{2}\right)+r_{1} \gamma_{1}+\cdots+r_{i} \gamma_{i}\right\rangle\right) \\
& \leq \frac{e_{i}}{r_{i+1}}\left(l-l_{i+1}(\nu)+r_{i+1}\left\langle\nu, \gamma_{i+1}\right\rangle\right) \\
& =\frac{e_{i}}{r_{i+1}} l-\left(\frac{e_{i}}{r_{i+1}}-1\right) l_{i+1}(\nu)
\end{aligned}
$$

Therefore $r_{i+1} l^{\prime} \leq e_{i} l-\left(e_{i}-r_{i+1}\right) l_{i+1}(\nu)$. Suppose that $l^{\prime} \geq l$, then

$$
\left(e_{i}-r_{i+1}\right) l_{i+1}(\nu) \leq e_{i} l-r_{i+1} l^{\prime} \leq\left(e_{i}-r_{i+1}\right) l
$$

and since $e_{i}>r_{i+1}$ it contradicts the fact that $j(l, \nu)=i$.

Now equation (18) follows by Lemma 3.12 .

From Corollary 4.15] we deduce the following:

$$
D_{m}^{\nu} \subset D\left(F_{0, \nu}^{\left(\frac{l_{1}(\nu)}{e_{0}}\right)} \cdots F_{j-1, \nu}^{\left(\frac{l_{j}(\nu)}{e_{j-1}}\right)}\right), \text { for any } \nu \in L_{m} \text { with } j(m, \nu)=j
$$

To illustrate the description of $J_{m}^{\nu}$ given in Corollary 4.15, we consider some particular cases.

- First, the simplest case, when $\nu \notin \rho_{1} \cup \rho_{2}$ we have $G_{j, \nu}=0$ for any $j$, and $m(\nu)=1$. Hence (note that we also have $c(\nu)=0)$ : 


$$
\begin{aligned}
& F_{0, \nu}^{(r)} \quad=z^{(r)}, \text { for } 0 \leq r<\frac{l_{1}(\nu)}{e_{0}} \\
& F_{1, \nu}^{\left(\frac{l_{1}(\nu)}{e_{1}}\right)} \quad=z^{\left(\left\langle\nu, \gamma_{1}\right\rangle\right)^{n_{1}}}-x_{1}^{\left(\nu_{1}\right)^{a_{1}}} \\
& F_{1}^{\left(\frac{l_{1}(\nu)}{e_{1}}+r\right)} \quad \text { for } 1 \leq r<\frac{l_{2}(\nu)-l_{1}(\nu)}{e_{1}}
\end{aligned}
$$

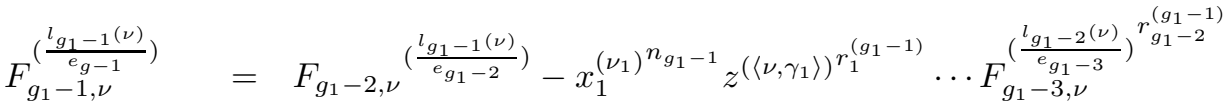

$$
\begin{aligned}
& F_{g_{1}-1, \nu}^{\left(\frac{l_{g_{1}-1}(\nu)}{e_{g}-1}+r\right)} \quad \text { for } 1 \leq r<\frac{l_{g_{1}}(\nu)-l_{g_{1}-1}(\nu)}{e_{g_{1}-1}}
\end{aligned}
$$

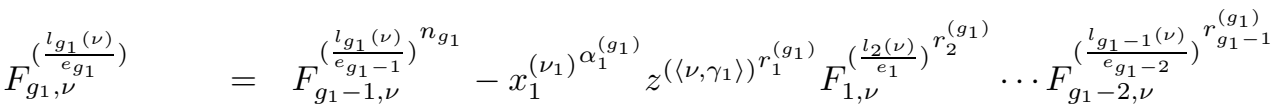

$$
\begin{aligned}
& F_{g_{1}, \nu}^{\left(\frac{l_{g_{1}}(\nu)}{e_{g_{1}}}+r\right)} \quad \text { for } 1 \leq r<\frac{l_{g_{1}+1}(\nu)-l_{g_{1}}(\nu)}{e_{g_{1}}}
\end{aligned}
$$

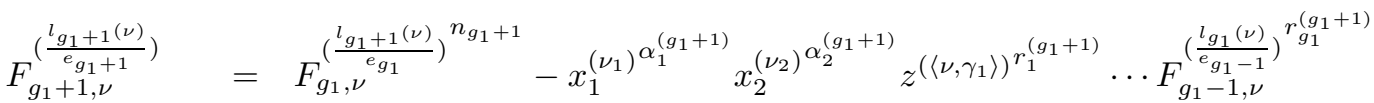

$$
\begin{aligned}
& \vdots \quad \vdots \\
& F_{j, \nu}^{\left(\frac{l_{j}(\nu)}{e_{j}}\right)} \quad=F_{j-1, \nu}^{\left(\frac{l_{j}(\nu)}{e_{j-1}}\right)^{n_{j}}}-x_{1}^{\left(\nu_{1}\right)^{\alpha_{1}^{(j)}}} x_{2}^{\left(\nu_{2}\right)^{\alpha_{2}^{(j)}}} z^{\left(\left\langle\nu, \gamma_{1}\right\rangle\right)^{r_{1}^{(j)}}} \cdots F_{j-2, \nu}^{\left(\frac{l_{j-1}(\nu)}{e_{j-2}}\right)^{r_{j-1}^{(j)}}} \\
& F_{j, \nu}^{\left(\frac{l_{j}(\nu)}{e_{j}}+r\right)} \quad \text { for } 1 \leq r \leq\left[\frac{m-l_{j}(\nu)}{e_{j}}\right]
\end{aligned}
$$

Notice that the variable $x_{2}^{\left(\nu_{2}\right)}$ appears for the first time in the equation $F_{g_{1}+1}^{\left(\frac{l_{g_{1}+1}(\nu)}{e_{g_{1}+1}}\right)}$, and raised to the power one or bigger depending on whether $g_{2}=g_{1}+1$ or $g_{2}=g_{1}$ respectively.

- Another example is when $j(m, \nu)=c(\nu)$, then

$$
J_{m}^{\nu}=\left(F_{c(\nu), \nu}^{(0)}, \ldots, F_{c(\nu), \nu}^{\left(\left[\frac{m}{\left.\left.e_{c(\nu)}\right]\right)}\right)\right.}\right.
$$

and if moreover $c(\nu)=0$ this is just the content of Proposition 4.12. Notice that the description of $J_{m}^{\nu}$ for $\nu \in H_{m}$ given in Proposition 4.12 is contained in Corollary 4.15, but we wanted to stress the fact that for $\nu \in H_{m}$ the description is particularly simple.

Now we can prove the irreducibility of the sets $C_{m}^{\nu}$.

Proposition 4.16. For any $m \in \mathbb{Z}_{>0}$ and $\nu \in H_{m} \cup L_{m}$, the set $C_{m}^{\nu}$ is irreducible and has codimension

$$
\nu_{1}+\nu_{2}+\sum_{k=0}^{j-1} \frac{l_{k+1}(\nu)-l_{k}(\nu)}{e_{k}}+\left[\frac{m-l_{j(m, \nu)}(\nu)}{e_{j(m, \nu)}}\right]+1
$$

Proof. To simplify notation we will denote along this proof $j(m, \nu)$ just by $j$ and by $k_{i}(\nu)$, or simply by $k_{i}$ when $\nu$ is clear from the context, we denote the quotient $\frac{l_{i+1}(\nu)-l_{i}(\nu)}{e_{i}}$. First notice that, by definition of $c(\nu), \sum_{k=0}^{j-1} \frac{l_{k+1}(\nu)-l_{k}(\nu)}{e_{k}}=\sum_{k=c(\nu)}^{j-1} \frac{l_{k+1}(\nu)-l_{k}(\nu)}{e_{k}}$.

If $\nu \in H_{m}$ we have $j(m, \nu)=0$ and the claim about the codimension follows by Proposition 4.12 The closed set $C_{m}^{\nu}$ is irreducible since it is defined by hyperplane coordinates. 
If $\nu \in L_{m}^{=}$, then by Lemma 4.18, we have that $C_{m}^{\nu}=V\left(I^{\nu}, J_{m}^{\nu}\right)$, where the ideal $J_{m}^{\nu}$ is described in equation (22). Then $\operatorname{Codim}\left(C_{m}^{\nu}\right)=\nu_{1}+\nu_{2}+\frac{l_{1}(\nu)}{n}+1$. Notice that if $c(\nu)>0$ then $l_{1}(\nu)=0$. The irreducibility of $C_{m}^{\nu}$ follows from the irreducibility of $F_{m(\nu), \nu}^{\left(\frac{l_{m(\nu)}(\nu)}{e_{m}(\nu)}\right)}$.

Now let $\nu$ be an element in $L_{m}^{<}$. We have to study carefully the generators of $J_{m}^{\nu}$ given in Corollary 4.15. Any $F_{i, \nu}^{(l)}$ is quasi-homogeneous of degree $l$, but the second property described in Remark 2.2 is not true anymore once we consider the equations modulo $I^{\nu}$. We need to know when a certain variable $x_{k}^{(l)}$ or $z^{(l)}$ appear for the first time in the generators of $J_{m}^{\nu}$. Notice that for any $\gamma \in D_{m}^{\nu}$ we have ord $\operatorname{lo}_{t}\left(x_{i} \circ \gamma\right)=\nu_{i}$ for $i=1,2$, and $\operatorname{ord}_{t}(z \circ \gamma)=\left\langle\nu, \gamma_{1}\right\rangle$. It is clear that the variables $x_{k}^{\left(\nu_{k}\right)}$ for $k=1,2$ and $z^{\left(\left\langle\nu, \gamma_{1}\right\rangle\right)}$ appear for the first time in the first non-monomial equation $F_{m(\nu), \nu}^{\left(\frac{l_{m(\nu)}(\nu)}{e_{m}(\nu)}\right)}$. In the next equation appear $x_{k}^{\left(\nu_{k}+1\right)}, z^{\left(\left\langle\nu, \gamma_{1}\right\rangle+1\right)}$, and so on. Then looking at the generators of $J_{m}^{\nu}$ described in Corollary 4.15, we deduce that, for $m(\nu) \leq i<j$ and $0 \leq r<k_{i}$, or $0 \leq r<\left[\frac{m-l_{j}(\nu)}{e_{j}}\right]$ when $i=j$,

$$
\text { the variables } x_{k}^{\left(\nu_{k}+k_{m(\nu)}+\cdots+k_{i-1}+r\right)}, z^{\left(\left\langle\nu, \gamma_{1}\right\rangle+k_{m(\nu)}+\cdots+k_{i-1}+r\right)} \text { appear for the first time in } F_{i, \nu}^{\left(\frac{l_{i}(\nu)}{e_{i}}+r\right)}
$$

Notice that for $1 \leq l<m(\nu)$ we have $k_{l}=0$. We divide the set of generators of $J_{m}^{\nu}$, given in Corollary 4.15, in two sets:

$$
\begin{aligned}
& \mathcal{C}_{1}=\left\{F_{i, \nu}^{\left(\frac{l_{i}(\nu)}{e_{i}}\right)}\right\}_{c(\nu) \leq i \leq j, l_{i}(\nu)<l_{i+1}(\nu)} \\
& \mathcal{C}_{2}=\left\{F_{i, \nu}^{\left(\frac{l_{i}(\nu)}{e_{i}}+r\right)}\right\}_{(i, r) \in A_{2}}
\end{aligned}
$$

where $A_{2}=\left\{(i, r) \mid c(\nu) \leq i<j, l_{i}(\nu)<l_{i+1}(\nu), 0<r<k_{i}\right\} \cup\left\{(j, r) \mid 0<r<\left[\frac{m-l_{j}(\nu)}{e_{j}}\right]\right\}$.

We claim:

(i) $V\left(\mathcal{C}_{1}\right) \simeq Z^{\Gamma_{m}^{\nu}}$, the toric variety defined by the semigroup $\Gamma_{m}^{\nu}$ generated by

$$
\left\{\gamma_{i}\right\}_{c(\nu) \leq i \leq j(m, \nu), l_{i}(\nu)<l_{i+1}(\nu)}
$$

If $\nu \notin \rho_{1} \cup \rho_{2}$ then $\Gamma_{m}^{\nu}=\Gamma_{j(m, \nu)}$ and $V\left(\mathcal{C}_{1}\right)$ is isomorphic to the monomial variety associated to $X^{(j(m, \nu))}$ (see Definition 3.6).

(ii) any $F_{i, \nu}^{\left(\frac{l_{i}(\nu)}{e_{i}}+r\right)} \in \mathcal{C}_{2}$ is linear over $D\left(x_{1}^{\left(\nu_{1}\right)}\right) \cap D\left(x_{2}^{\left(\nu_{2}\right)}\right)$ with respect to one of the variables described in $(*)$, which appears for the first time on this equation.

Since any of these equations in $\mathcal{C}_{2}$ is linear in a different variable, and, by $(*)$ we have that it appears for the first time in $\mathcal{C}_{2}$, we deduce

$$
V\left(I^{\nu}, \mathcal{C}_{2}\right) \cap D\left(x_{1}^{\left(\nu_{1}\right)}\right) \cap D\left(x_{2}^{\left(\nu_{2}\right)}\right) \simeq \mathbb{A}^{\alpha(m, \nu)}
$$

where $\alpha(m, \nu)=3(m+1)-\nu_{1}-\nu_{2}-\sum_{i=c(\nu), \ldots, j-1, l_{i}(\nu)<l_{i+1}(\nu)}\left(k_{i}-1\right)-\left[\frac{m-l_{j}(\nu)}{e_{j}}\right]$, because $V\left(I^{\nu}, \mathcal{C}_{2}\right) \subseteq$ $\mathbb{A}_{m}^{3} \simeq \mathbb{A}^{3(m+1)}$. Hence

$$
D_{m}^{\nu} \simeq\left(Z^{\Gamma_{m}^{\nu}} \cap D\left(x_{1}^{\left(\nu_{1}\right)}\right) \cap D\left(x_{2}^{\left(\nu_{2}\right)}\right)\right) \times \mathbb{A}^{\alpha(m, \nu)}
$$

The toric variety $Z^{\Gamma_{m}^{\nu}}$ is irreducible and hence the irreducibility of $C_{m}^{\nu}$ follows by the previous isomorphism. Moreover $Z^{\Gamma_{m}^{\nu}}$ is complete intersection, hence the codimension equals the number of defining equations, which is the cardinal of $\mathcal{C}_{1}$. Therefore

$$
\begin{aligned}
\operatorname{Codim}\left(C_{m}^{\nu}\right) & =\sharp \mathcal{C}_{1}+\nu_{1}+\nu_{2}+\sum_{c(\nu) \leq i<j, l_{i}(\nu)<l_{i+1}(\nu)}\left(k_{i}-1\right)+\left[\frac{m-l_{j}(\nu)}{e_{j}}\right] \\
& =\nu_{1}+\nu_{2}+\sum_{c(\nu) \leq i<j, l_{i}(\nu)<l_{i+1}(\nu)} k_{i}+\left[\frac{m-l_{j}(\nu)}{e_{j}}\right]+\sharp \mathcal{C}_{1}-\sharp\left\{c(\nu) \leq i<j \mid l_{i}(\nu)<l_{i+1}(\nu)\right\}
\end{aligned}
$$

Finally the statement about the codimension follows now by these two remarks:

- $\sum_{c(\nu) \leq i<j, l_{i}(\nu)<l_{i+1}(\nu)} k_{i}=\sum_{i=c(\nu)}^{j-1} k_{i}$, since $k_{i}=0$ whenever $l_{i}(\nu)=l_{i+1}(\nu)$.

- $l_{j}(\nu)<l_{j+1}(\nu)$ by definition of $j(m, \nu)$, and therefore

$$
\sharp\left\{c(\nu) \leq i<j \mid l_{i}(\nu)<l_{i+1}(\nu)\right\}=\sharp \mathcal{C}_{1}-1 .
$$


Now we prove the claim. To prove (i), notice that we can write equation (18) as

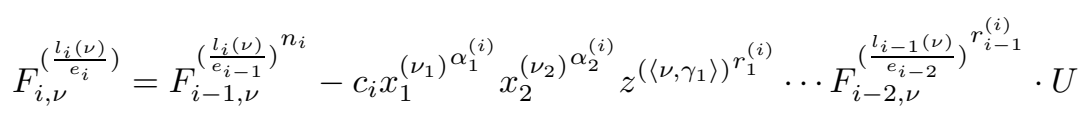

where, arguing as in the proof of Lemma 5.4 (i), we have $U \neq 0$. Then we have the isomorphism

$$
V\left(F_{i, \nu}^{\left(\frac{l_{i}(\nu)}{e_{i}}\right)}\right)_{c(\nu) \leq i \leq j(m, \nu), l_{i}(\nu)<l_{i+1}(\nu)} \simeq V\left(h_{i}\right)_{c(\nu) \leq i \leq j(m, \nu), l_{i}(\nu)<l_{i+1}(\nu)}
$$

where $h_{i}=w_{i}^{n_{i}}-x_{1}^{\alpha_{1}^{(i)}} x_{2}^{\alpha_{2}^{(i)}} z_{1}^{r_{1}^{(i)}} w_{1}^{r_{2}^{(i)}} \cdots w_{i-2}^{r_{i-1}^{(i)}}$, with the relation $n_{i} \gamma_{i}=\left(\alpha_{1}^{(i)}, \alpha_{2}^{(i)}\right)+r_{1}^{(i)} \gamma_{1}+\cdots+r_{i-1}^{(i)} \gamma_{i-1}$. And $V\left(h_{i}\right)_{c(\nu) \leq i \leq j(m, \nu), l_{i}(\nu)<l_{i+1}(\nu)}$ is isomorphic to the toric variety $Z^{\Gamma_{m}^{\nu}}$.

To prove the claim (ii), we distinguish three cases, depending on $m$.

(a) For $m<l_{g_{1}+1}(\nu)+e_{g_{1}+1}$. In this case we have that $g_{1}>0$ and therefore $m(\nu)$ is either $g_{1}$ or 1 . Suppose first that $m(\nu)=1$. Then

$$
F_{1, \nu}^{\left(\frac{l_{1}(\nu)}{e_{1}}\right)}=z^{\left(\left\langle\nu, \gamma_{1}\right\rangle\right)^{n_{1}}}-x_{1}^{\left(\nu_{1}\right)^{a_{1}}}+G_{1, \nu},
$$

where $G_{1, \nu}$ is the polynomial $\sum c_{i_{1} i_{2} k} x_{1}^{\left(\nu_{1}\right)^{i_{1}}} x_{2}^{\left(\nu_{2}\right)^{i_{2}}} z^{\left(\left\langle\nu, \gamma_{1}\right\rangle\right)^{k}}$ with $\left\langle\nu,\left(i_{1}, i_{2}\right)+k \gamma_{1}\right\rangle=n_{1}\left\langle\nu, \gamma_{1}\right\rangle=a_{1} \nu_{1}$. Moreover, for $0<r<k_{1}, F_{1, \nu}^{\left(\frac{l_{1}(\nu)}{e_{1}}+r\right)}$ is a quasi-homogeneous polynomial of degree $\frac{l_{1}(\nu)}{e_{1}}+r=a_{1} \nu_{1}+r$, and using $(*)$

$$
F_{1, \nu}^{\left(\frac{l_{1}(\nu)}{e_{1}}+r\right)}=x_{1}^{\left(\nu_{1}\right)^{a_{1}-1}} x_{1}^{\left(\nu_{1}+r\right)} \cdot U_{1}^{(r)}+H_{1, \nu}^{\left(\frac{l_{1}(\nu)}{e_{1}}+r\right)}
$$

where $H_{1, \nu}^{\left(\frac{l_{1}(\nu)}{e_{1}}+r\right)}$ is a polynomial in which $x_{1}^{\left(\nu_{1}+r\right)}$ do not appear, and $U_{1}^{(r)} \neq 0$. Analogously, for $1<i<$ $j(m, \nu)$ and $0<r<k_{i}(\nu)$ we have

$$
F_{i, \nu}^{\left(\frac{l_{i}(\nu)}{e_{i}}+r\right)}=n_{i} F_{i-1, \nu}^{\left(\frac{l_{i}(\nu)}{e_{i-1}}\right)^{n_{i}-1}} F_{i-1, \nu}^{\left(\frac{l_{i}(\nu)}{e_{i}-1}+r\right)}+H_{i, \nu}^{\left(\frac{l_{i}(\nu)}{e_{i}}+r\right)}
$$

where $H_{i, \nu}^{\left(\frac{l_{i}(\nu)}{e_{i}}+r\right)}$ is a polynomial in which the variable $x_{1}^{\left(\nu_{1}+k_{1}+\cdots+k_{i-1}+r\right)}$ does not appear. Moreover

$$
F_{i-1, \nu}^{\left(\frac{l_{i}(\nu)}{e_{i-1}}+r\right)}=a_{1} x_{1}^{\left(\nu_{1}\right)^{a_{1}-1}} x_{1}^{\left(\nu_{1}+k_{1}+\cdots+k_{i-1}+r\right)}+H_{i-1, \nu}^{(r)},
$$

where the variable $x_{1}^{\left(\nu_{1}+k_{1}+\cdots+k_{i-1}+r\right)}$ does not appear in the polynomial $H_{i-1, \nu}^{(r)}$. Then, by Lemma 5.4 (ii) it follows that for $1=m(\nu) \leq i<j(m, \nu)$ and $0<r<k_{i}(\nu)$ the equation $F_{i}^{\left(\frac{l_{i}(\nu)}{e_{i}}+r\right)}$ is linear on $x_{1}^{\left(\nu_{1}+k_{1}+\cdots+k_{i-1}+r\right)}$ over $D\left(x_{1}^{\left(\nu_{1}\right)}\right)$. We still have to deal with the equations $F_{j}^{\left(\frac{l_{j}(\nu)}{e_{j}}+r\right)}$ with $1 \leq r \leq\left[\frac{m-l_{j}(\nu)}{e_{j}}\right]$. Notice that $j(m, \nu) \leq g_{1}+1$. If $j(m, \nu)<g_{1}+1$ then the argument is exactly as before, and if $j(m, \nu)=g_{1}+1$ then $\left[\frac{m-l_{j}(\nu)}{e_{j}}\right]=0$.

Suppose now that $m(\nu)=g_{1}>1$. Then $\nu=\left(0, \nu_{2}\right)$ and the generators of $J_{m}^{\nu}$ are

$$
\begin{array}{ll}
F_{g_{1}}^{(r)} \text { for } 0 \leq r \leq\left[\frac{m-l_{g_{1}}(\nu)}{e_{g_{1}}}\right] & \text { if } j(m, \nu)=g_{1} \\
F_{g_{1}}^{(r)} \text { for } 0 \leq r<k_{g_{1}}(\nu) \text { and } F_{g_{1}+1}^{\left(\frac{l_{g_{1}+1}(\nu)}{e_{g_{1}+1}}\right)} & \text { if } j(m, \nu)=g_{1}+1
\end{array}
$$

For $r>0$ we have that $F_{g_{1}}^{(r)} \equiv \frac{\partial F_{g_{1}}}{\partial x_{1}}\left(x_{1}^{(0)}, z^{(0)}\right) x_{1}^{(r)}+\frac{\partial F_{g_{1}}}{\partial z}\left(x_{1}^{(0)}, z^{(0)}\right) z^{(0)}+H_{r} \bmod I^{\nu}$, with $H_{r}$ a polynomial where the variables $x_{1}^{(r)}$ and $z^{(r)}$ do not appear. Since we are looking at $\left\{x_{1}^{(0)} \neq 0\right\}$, we are outside the singular locus, and we deduce that these equations are linear either in $x_{1}^{(r)}$ or in $z^{(r)}$. The rest of the proof follows as in the previous part of this case.

(b) For $l_{g_{1}+1}(\nu)+e_{g_{1}+1} \leq m<l_{g_{2}+1}(\nu)+e_{g_{2}+1}$. This is only possible when $g_{2}=g_{1}+1$. Notice that if $j(m, \nu)=g_{2}+1$ then $\left[\frac{m-l_{j}(\nu)}{e_{j}}\right]=0$. We just have to study the generators $F_{i, \nu}^{\left(\frac{l_{i}(\nu)}{e_{i}}+r\right)}$ for $i>g_{1}$ (which are all the generators of $J_{m}^{\nu}$ when $\left.g_{1}=0\right)$ since the others were studied in the previous case. That is, we have 
the equations $F_{g_{2}, \nu}^{\left(\frac{l_{g_{2}}(\nu)}{g_{2}}+r\right)}$ for $0 \leq r \leq k_{g_{2}}(\nu)-1$ if $j(m, \nu)=g_{2}+1$ and $0 \leq r \leq\left[\frac{m-l_{g_{2}}(\nu)}{e_{g_{2}}}\right]$ otherwise. If

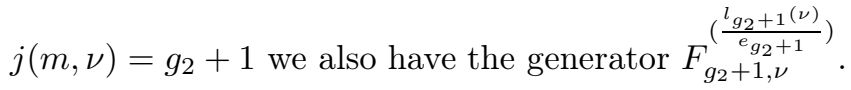

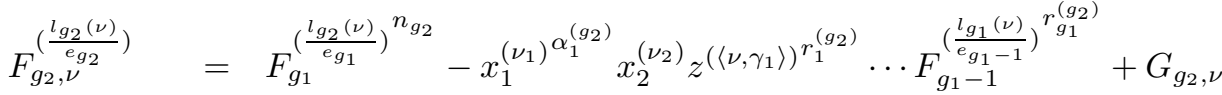

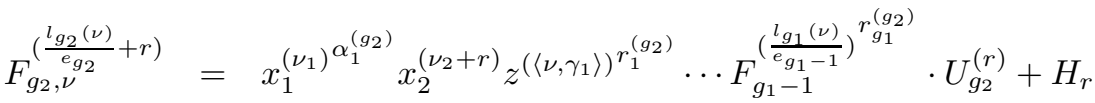

where $U_{g_{2}}^{(r)}$ is 1 if $\nu \notin \rho_{1} \cup \rho_{2}$ and $\neq 0$ otherwise, and $H_{r}$ is a polynomial in which the variable $x_{2}^{\left(\nu_{2}+r\right)}$ does not appear. Then by Lemma 5.4 (ii) we deduce that every $F_{g_{2}, \nu}^{\left.\frac{\left(l_{g_{2}(\nu)} g_{2}\right.}{2}+r\right)}$ is linear on $x_{2}^{\left(\nu_{2}+r\right)}$ over $D\left(x_{1}^{\left(\nu_{1}\right)}\right)$. And the rest of the argument goes as in the previous case.

(c) For $l_{g_{2}+1}(\nu)+e_{g_{2}+1} \leq m$, with the same arguments it is easy to see that for $i>g_{2}$ and $1 \leq r<k_{i}(\nu)$ each $F_{i, \nu}^{\left(\frac{l_{i}(\nu)}{e_{i}}+r\right)}$ is linear on $x_{1}^{\left(\nu_{1}+k_{1}+\cdots+k_{i}+r\right)}$ over $D\left(x_{1}^{\left(\nu_{1}\right)}\right) \cap D\left(x_{2}^{\left(\nu_{2}\right)}\right)$.

In particular we have the following variation of the codimension of $C_{m}^{\nu}$ as $m$ grows.

Corollary 4.17. For $\nu \in H_{m} \cup L_{m}$ such that $\nu \in H_{m-1} \cup L_{m-1}$ we have that

$$
\operatorname{Codim}\left(C_{m}^{\nu}\right)= \begin{cases}\operatorname{Codim}\left(C_{m-1}^{\nu}\right)+1 & \text { if } m \equiv 0 \bmod e_{j(m-1, \nu)} \\ \operatorname{Codim}\left(C_{m-1}^{\nu}\right) & \text { otherwise }\end{cases}
$$

4.2. Inclusions among the $C_{m}^{\nu}$. We have a collection of irreducible sets $\left\{C_{m}^{\nu} \mid \nu \in H_{m} \cup L_{m}\right\}$ covering $\pi_{m}^{-1}\left(X_{\text {Sing }}\right)$, but in general it is not its decomposition in irreducible components. We have to study the inclusions

$$
C_{m}^{\nu^{\prime}} \subseteq C_{m}^{\nu} \text { for different } \nu, \nu^{\prime} \in H_{m} \cup L_{m} .
$$

We need to see $C_{m}^{\nu}$ as the closure of a set, which is slightly different from $D_{m}^{\nu}$, though described by the ideals $I^{\nu}$ and $J_{m}^{\nu}$. For instance, when $j(m, \nu)=c(\nu)=g_{1}>0$, by Corollary 4.15 $J_{m}^{\nu}=\left(F_{g_{1}, \nu}^{(0)}, \ldots, F_{g_{1}, \nu}^{\left(\left[\frac{m}{e_{1}}\right]\right)}\right)$, and

$$
C_{m}^{\nu}=\overline{V\left(I^{\nu}, F_{g_{1}, \nu}^{(0)}, \ldots, F_{g_{1}, \nu}^{\left(\left[\frac{m}{e_{1}}\right]\right)}\right) \cap D\left(x_{1}^{\left(\nu_{1}\right)}\right)},
$$

because the polynomials $F_{g_{1}, \nu}^{(0)}, \ldots, F_{g_{1}, \nu}^{\left(\left[\frac{m}{e_{1}}\right]\right)}$ do not depend on $x_{2}^{\left(\nu_{2}\right)}$, and hence, when taking the Zariski closure, we can drop the condition $D\left(x_{2}^{\left(\nu_{2}\right)}\right)$ in the description of $D_{m}^{\nu}$ given in (16). This is the description we are looking for, and it is the content of the next Lemma.

Lemma 4.18. For $m \in \mathbb{Z}_{>0}$ and $\nu \in H_{m} \cup L_{m}$ we have that $C_{m}^{\nu}=\overline{O_{m}^{\nu}}$, where

$$
O_{m}^{\nu}:=\left\{\begin{array}{cl}
V\left(I^{\nu}, J_{m}^{\nu}\right) & \text { if } j^{\prime}(m, \nu)<m(\nu) \\
V\left(I^{\nu}, J_{m}^{\nu}\right) \cap D\left(x_{1}^{\left(\nu_{1}\right)}\right) & \text { if } m(\nu) \leq j^{\prime}(m, \nu) \leq g_{2} \\
V\left(I^{\nu}, J_{m}^{\nu}\right) \cap D\left(x_{1}^{\left(\nu_{1}\right)}\right) \cap D\left(x_{2}^{\left(\nu_{2}\right)}\right) & \text { if } j^{\prime}(m, \nu) \geq g_{2}+1
\end{array}\right.
$$

Notice that when $j^{\prime}(m, \nu)<m(\nu)$ then $O_{m}^{\nu}=C_{m}^{\nu}$, and if $j^{\prime}(m, \nu) \geq g_{2}+1$ then $O_{m}^{\nu}=D_{m}^{\nu}$.

Proof. If $j^{\prime}(m, \nu)<m(\nu)$ we have two possibilities regarding $j(m, \nu)$, either $j(m, \nu)<m(\nu)$ or $j(m, \nu)=$ $m(\nu)$. Suppose that $j(m, \nu)<m(\nu)$, then $j(m, \nu)=0$, since $l_{j(m, \nu)}(\nu)<l_{j(m, \nu)+1}(\nu)$ and $l_{1}(\nu)=\cdots=$ $l_{m(\nu)}(\nu)<l_{m(\nu)+1}(\nu)$. Then $\nu \in H_{m}$, and by Proposition 4.12 we have that $C_{m}^{\nu}=V\left(I^{\nu}, J_{m}^{\nu}\right)$ where $J_{m}^{\nu}=\left(z^{(0)}, \ldots, z^{\left(\left[\frac{m}{n}\right]\right)}\right)$. If $j(m, \nu)=m(\nu)$ we have that $J_{m}^{\nu}$ is the ideal

$$
J_{m}^{\nu}=\left\{\begin{array}{cl}
\left(F_{g_{1}, \nu}^{(0)}\right) & \text { if } c(\nu)=g_{1}>0 \\
\left(z^{(0)}, \ldots, z^{\left(\left\langle\nu, \gamma_{1}\right\rangle-1\right)}, F_{m(\nu), \nu}^{\left(\frac{l_{m(\nu)} e_{m}(\nu)}{e^{\prime}}\right)}\right) & \text { otherwise }
\end{array}\right.
$$


Therefore the conditions $D\left(x_{1}^{\left(\nu_{1}\right)}\right) \cap D\left(x_{2}^{\left(\nu_{2}\right)}\right)$ disappear when taking the Zariski closure.

Suppose now that $m(\nu) \leq j^{\prime}(m, \nu) \leq g_{2}$. We prove that

$$
\overline{V\left(I^{\nu}, J_{m}^{\nu}\right) \cap D\left(x_{1}^{\left(\nu_{1}\right)}\right) \cap D\left(x_{2}^{\left(\nu_{2}\right)}\right)}=\overline{V\left(I^{\nu}, J_{m}^{\nu}\right) \cap D\left(x_{1}^{\left(\nu_{1}\right)}\right)}
$$

or in other words, the open condition $x_{2}^{\left(\nu_{2}\right)} \neq 0$ is superfluous when taking the Zariski closure. This claim is obvious for $m(\nu) \leq j^{\prime}(m, \nu) \leq g_{1}$, since $x_{2}^{\left(\nu_{2}\right)}$ appears in the generators of $J_{m}^{\nu}$ at most once (in $F_{g_{1}+1, \nu}^{\left(\frac{l_{g_{1}+1}(\nu)}{g_{1}+1}\right)}$ if $\left.l_{g_{1}+1}(\nu) \leq m<l_{g_{1}+1}(\nu)+e_{g_{1}+1}\right)$. For $g_{1}<j^{\prime}(m, \nu) \leq g_{2}$, we are necessarily in the case $g_{2}=g_{1}+1$ and we prove the equality (23) by induction on $m$. For $m=l_{g_{2}}(\nu)+e_{g_{2}}$, if the equality (23) does not hold, then

$$
C:=\overline{V\left(I^{\nu}, J_{m}^{\nu}\right) \cap D\left(x_{1}^{\left(\nu_{1}\right)}\right) \cap\left\{x_{2}^{\left(\nu_{2}\right)}=0\right\}} \nsubseteq C_{m}^{\nu}
$$

Notice that by Corollary 4.15

$$
F_{g_{2}, \nu}^{\left(\frac{l_{g_{2}(\nu)}}{g_{2}}\right)}=F_{g_{2}-1, \nu}^{\left(\frac{l_{g_{2}(\nu)}}{e_{2}-1}\right)^{n_{g_{2}}}}-c_{g_{2}} x_{1}^{\left(\nu_{1}\right)^{\alpha_{1}^{\left(g_{2}\right)}}} x_{2}^{\left(\nu_{2}\right)} \cdots F_{g_{2}-2, \nu}^{\left(\frac{l_{g_{2}-1}(\nu)}{e_{2}-2}\right)^{r_{g_{2}-1}^{\left(g_{2}\right)}}}+G_{g_{2}, \nu}
$$

and by Lemma 5.4 (i) we deduce that, if $x_{2}^{\left(\nu_{2}\right)}=0$ then $F_{g_{2}-1, \nu}^{\left(\frac{l_{g_{2}}(\nu)}{g_{2}-1}\right)}$. Moreover, since the polynomials $F_{\nu}^{(l)}$ are quasi-homogeneous

$$
F_{g_{2}, \nu}^{\left(\frac{l_{g_{2}}(\nu)}{g_{2}}+1\right)} \equiv \bar{c}_{g_{2}} x_{1}^{\left(\nu_{1}\right)^{\left(g_{2}\right)}} x_{2}^{\left(\nu_{2}+1\right)} \cdots F_{g_{2}-2, \nu}^{\left(\frac{l_{g_{2}-1}(\nu)}{e_{2}-2}\right)^{r_{g_{2}-1}^{\left(g_{2}\right)}}} \bmod x_{2}^{\left(\nu_{2}\right)}
$$

and, by (19), we deduce that $x_{2}^{\left(\nu_{2}+1\right)}=0$. Hence

$$
C=V\left(I^{\nu}, J_{l_{g_{2}}(\nu)-1}^{\nu}, x_{2}^{\left(\nu_{2}\right)}, x_{2}^{\left(\nu_{2}+1\right)}, F_{g_{2}-1, \nu}^{\left(\frac{l_{g_{2}}(\nu)}{g_{2}-1}\right)}\right)
$$

and now consider the closed set $C^{\prime}:=\pi_{m, m-1}\left(C_{m}^{\nu}\right)=\overline{V\left(I^{\nu}, J_{m-1}^{\nu}\right) \cap D\left(x_{1}^{\left(\nu_{1}\right)}\right)}$. We have that $\pi_{m, m-1}^{-1}\left(C^{\prime}\right)=$ $\overline{V\left(I^{\nu}, J_{m}^{\nu}\right) \cap D\left(x_{1}^{\left(\nu_{1}\right)}\right)}=C_{m}^{\nu} \cup C$ with $\operatorname{Codim}\left(C_{m}^{\nu}\right)=\operatorname{Codim}\left(C^{\prime}\right)+1$ and $\operatorname{Codim}(C)=\operatorname{Codim}\left(C^{\prime}\right)+2$, which is a contradiction. Suppose it true for $m$ and we prove it for $m+1$. Consider $C^{\prime}:=\pi_{m+1, m}\left(C_{m}^{\nu}\right)=$ $\overline{V\left(I^{\nu}, J_{m}^{\nu}\right) \cap D\left(x_{1}^{\left(\nu_{1}\right)}\right) \cap D\left(x_{2}^{\left(\nu_{2}\right)}\right)}$. By induction hypothesis $C^{\prime}=\overline{V\left(I^{\nu}, J_{m}^{\nu}\right) \cap D\left(x_{1}^{\left(\nu_{1}\right)}\right)}$, and then $\pi_{m+1, m}^{-1}\left(C^{\prime}\right)=$ $\overline{V\left(I^{\nu}, J_{m}^{\nu}, F_{\nu}^{(m+1)}\right) \cap D\left(x_{1}^{\left(\nu_{1}\right)}\right)}$. If $F_{\nu}^{(m+1)}=0$ then we are done. Otherwise, by Corollary 4.15, $F_{\nu}^{(m+1)}=$ $F_{g_{2}, \nu}^{\left(\frac{l_{g_{2}}(\nu)}{e_{g_{2}}}+r\right)}{ }^{e_{g_{2}}}$ where $r=\frac{m+1-l_{g_{2}}(\nu)}{e_{g_{2}}}$, and, as in the first step of induction, if it subdivides as

$$
C_{m+1}^{\nu} \cup \overline{V\left(I^{\nu}, J_{m}^{\nu}, F_{\nu}^{(m+1)}\right) \cap D\left(x_{1}^{\left(\nu_{1}\right)}\right) \cap\left\{x_{2}^{\left(\nu_{2}\right)}=0\right\}},
$$

then $\operatorname{Codim}\left(\overline{V\left(I^{\nu}, J_{m}^{\nu}, F_{\nu}^{(m+1)}\right) \cap D\left(x_{1}^{\left(\nu_{1}\right)}\right) \cap\left\{x_{2}^{\left(\nu_{2}\right)}=0\right\}}\right)=\operatorname{Codim}\left(C^{\prime}\right)+2$ which is a contradiction.

Finally, if $j^{\prime}(m, \nu) \geq g_{2}+1$ there is nothing to prove.

We will describe a set $F_{m} \subset H_{m} \cup L_{m}$ such that $\left\{C_{m}^{\nu} \mid \nu \in F_{m}\right\}$ is the set of irreducible components. The process of defining $F_{m}$ as a subset of $H_{m} \cup L_{m}$ is done in two steps. The first reduction is easy. We consider the product ordering $\leq_{p}$ in $\mathbb{Z}^{2}$ given by:

$$
\nu \leq_{p} \nu^{\prime} \text { if and only if } \nu_{i} \leq \nu_{i}^{\prime} \text { for } i=1,2 .
$$

Proposition 4.19. For $\nu, \nu^{\prime} \in H_{m} \cup L_{m}$ we have that

(i) If $C_{m}^{\nu^{\prime}} \subseteq C_{m}^{\nu}$ then $\nu \leq_{p} \nu^{\prime}$.

(ii) Moreover if $\nu, \nu^{\prime} \in H_{m} \cup L_{m}^{=}$then we have

$$
C_{m}^{\nu^{\prime}} \subseteq C_{m}^{\nu} \Longleftrightarrow \nu \leq{ }_{p} \nu^{\prime}
$$

Proof.

(i) Suppose that $\nu$ and $\nu^{\prime}$ are not comparable. Then we can assume that $\nu_{1}\left\langle\nu_{1}^{\prime}\right.$ and $\nu_{2}>\nu_{2}^{\prime}$. Then, since $C_{m}^{\nu} \subseteq V\left(I^{\nu}\right)$, and $C_{m}^{\nu^{\prime}} \subset V\left(I^{\nu^{\prime}}\right)$, it follows that

$$
C_{m}^{\nu} \nsubseteq C_{m}^{\nu^{\prime}} \text { and } C_{m}^{\nu^{\prime}} \nsubseteq C_{m}^{\nu}
$$


(ii) The claim follows by (i) and the definition of $C_{m}^{\nu}$ for $\nu \in H_{m} \cup L_{m}^{=}$.

Definition 4.20. According to Proposition 4.19 we define the set:

$$
P_{m}=\min _{\leq p}\left\{H_{m} \cup L_{m}^{=}\right\} .
$$

The second reduction, which defines the set $F_{m} \subseteq P_{m} \cup L_{m}^{<}$, is much more involved, and the singular locus of the approximated roots play a role now when studying the inclusions $C_{m}^{\nu^{\prime}} \subseteq C_{m}^{\nu}$ for different elements $\nu$ and $\nu^{\prime}$ in $P_{m} \cup L_{m}^{<}$. By Proposition 4.19 (i) we have to consider $\nu \Varangle_{p} \nu^{\prime}$, where, by definition of $P_{m}, \nu \in L_{m}^{<}$ and $\nu^{\prime} \in P_{m} \cup L_{m}^{<}$.

Proposition 4.21. Given $m \in \mathbb{Z}_{>0}, \nu \in L_{m}^{<}$and $\nu^{\prime} \in P_{m} \cup L_{m}^{<}$with $\nu \lesseqgtr p$, such that $\nu^{\prime}-\nu \in \sigma_{R e g, j^{\prime}(m, \nu)}$ then $C_{m}^{\nu^{\prime}} \subseteq C_{m}^{\nu}$.

Proof. We simplify notation by setting $k_{i}(\nu)=\frac{l_{i+1}(\nu)-l_{i}(\nu)}{e_{i}}$, for $1 \leq i \leq g$. By the description of $\sigma_{R e g, j}$ given in (14) we have to prove the inclusion $C_{m}^{\nu^{\prime}} \subseteq C_{m}^{\nu}$ when $\nu^{\prime}-\nu \in \sigma_{R e g, j^{\prime}(m, \nu)}$ and $1 \leq j^{\prime}(m, \nu) \leq g_{2}$. Then, by Lemma 4.18, we have that

$$
C_{m}^{\nu}=\overline{V\left(I^{\nu}, J_{m}^{\nu}\right) \cap D\left(x_{1}^{\left(\nu_{1}\right)}\right)}
$$

Suppose first that $\sigma_{R e g, j^{\prime}(m, \nu)}=\rho_{2}$, then $\nu^{\prime}=\nu+(0, \beta)$ with $\beta>0$. We distinguish two cases:

(i) If $\nu^{\prime} \in H_{m}$, then by Proposition 4.12 ,

$$
C_{m}^{\nu^{\prime}}=V\left(I^{\nu}, x_{2}^{\left(\nu_{2}\right)}, \ldots, x_{2}^{\left(\nu_{2}+\beta-1\right)}, z^{(0)}, \ldots, z^{([m / n])}\right) .
$$

Note that $g_{1}=0$, because otherwise $l_{1}\left(\nu^{\prime}\right)=l_{1}(\nu)$ and $\nu^{\prime} \notin H_{m}$. Then, since $1 \leq j^{\prime}(m, \nu) \leq g_{2}$, we deduce that $g_{2}=1$. There exists $1 \leq r<k_{1}(\nu)$ such that

$$
l_{1}(\nu)+r e_{1} \leq m<l_{1}(\nu)+(r+1) e_{1}
$$

since $\nu \in L_{m}^{<}$. Then

$$
J_{m}^{\nu}=\left(z^{(0)}, \ldots, z^{\left(\left\langle\nu, \gamma_{1}\right\rangle-1\right)}, F_{1, \nu}^{\left(\frac{l_{1}(\nu)}{e_{1}}\right)}, \ldots, F_{1, \nu}^{\left(\frac{l_{1}(\nu)}{e_{1}}+r\right)}\right) .
$$

Notice that $\left[\frac{m}{n}\right]=\left[\frac{l_{1}(\nu)+r e_{1}}{n}\right]=\left\langle\nu, \gamma_{1}\right\rangle+\alpha$, where $\alpha=\left[\frac{r}{n_{1}}\right]$. Now, since $\nu^{\prime}=\nu+(0, \beta) \in H_{m}$ and $g_{2}=1$, we have that $l_{1}\left(\nu^{\prime}\right)=l_{1}(\nu)+e_{1} \beta \geq m+1$ and it follows that $\beta>r$. Hence we have to prove

$$
F_{1, \nu}^{\left(\frac{l_{1}(\nu)}{e_{1}}+l\right)} \equiv 0 \bmod \left(x_{2}^{\left(\nu_{2}\right)}, \ldots, x_{2}^{\left(\nu_{2}+\beta-1\right)}, F_{1, \nu}^{\left(\frac{l_{1}(\nu)}{e_{1}}\right)}, \ldots, F_{1, \nu}^{\left(\frac{l_{1}(\nu)}{e_{1}}+l-1\right)}\right)
$$

for $0 \leq l \leq r$. By Corollary 4.15 we have $F_{1, \nu}^{\left(\frac{l_{1}(\nu)}{e_{1}}\right)}=z^{\left(\left\langle\nu, \gamma_{1}\right\rangle\right)^{n_{1}}}-x_{1}^{\left(\nu_{1}\right)^{a_{1}}} x_{2}^{\left(\nu_{2}\right)}+G_{1, \nu}$. And by Lemma 5.4 (i), if $x_{2}^{\left(\nu_{2}\right)}=0$ then $z^{\left(\left\langle\nu, \gamma_{1}\right\rangle\right)}=0$, and hence $G_{1, \nu}=0$.

By quasi-homogeneity we can write

$$
F_{1, \nu}^{\left(\frac{l_{1}(\nu)}{e_{1}}+1\right)}=c_{1} z^{\left(\left\langle\nu, \gamma_{1}\right\rangle\right)^{n_{1}-1}} z^{\left(\left\langle\nu, \gamma_{1}\right\rangle+1\right)}+c_{2} x_{1}^{\left(\nu_{1}\right)^{a_{1}-1}} x_{1}^{\left(\nu_{1}+1\right)} x_{2}^{\left(\nu_{2}\right)}+c_{3} x_{1}^{\left(\nu_{1}\right)^{a_{1}}} x_{2}^{\left(\nu_{2}+1\right)}+G_{1, \nu}^{(1)}
$$

where $c_{1}, c_{2}, c_{3}$ are certain coefficients and $G_{1, \nu}^{(1)}$ is a quasi-homogeneous polynomial of degree $\frac{l_{1}(\nu)}{e_{1}}+1$. We have that $G_{1, \nu}^{(1)}=0$ when $\nu \notin \rho_{1} \cup \rho_{2}$, and otherwise, we can apply the same arguments as in the proof of Lemma 5.4 we deduce that

$$
F_{1, \nu}^{\left(\frac{l_{1}(\nu)}{e}+1\right)} \equiv 0 \bmod \left(x_{2}^{\left(\nu_{2}\right)}, x_{2}^{\left(\nu_{2}+1\right)}, z^{\left(\left\langle\nu, \gamma_{1}\right\rangle\right)}\right) .
$$

Again by quasi-homogeneity, if $n_{1}<r$,

$$
\begin{aligned}
F_{1, \nu}^{\left(\frac{l_{1}(\nu)}{e_{1}}+n_{1}\right)}= & z^{\left(\left\langle\nu, \gamma_{1}\right\rangle\right)} h_{1,0}\left(z^{\left(\left\langle\nu, \gamma_{1}\right\rangle\right)}, \ldots, z^{\left(\left\langle\nu, \gamma_{1}\right\rangle+n_{1}\right)}\right)+z^{\left(\left\langle\nu, \gamma_{1}\right\rangle+1\right)^{n_{1}}}+x_{2}^{\left(\nu_{2}\right)} h_{2,0}\left(x_{1}^{\left(\nu_{1}\right)}, \ldots, x_{1}^{\left(\nu_{1}+n_{1}\right)}\right)+ \\
& x_{2}^{\left(\nu_{2}+1\right)} h_{2,1}\left(x_{1}^{\left(\nu_{1}\right)}, \ldots, x_{1}^{\left(\nu_{1}+n_{1}\right)}\right)+\cdots+x_{2}^{\left(\nu_{2}+n_{1}\right)} h_{2, n_{1}}\left(x_{1}^{\left(\nu_{1}\right)}, \ldots, x_{1}^{\left(\nu_{1}+n_{1}\right)}\right)+G_{1, \nu}^{\left(n_{1}\right)} .
\end{aligned}
$$

And analogously we prove that

$$
F_{1, \nu}^{\left(\frac{l_{1}(\nu)}{e_{1}}+n_{1}\right)} \equiv 0 \bmod \left(x_{2}^{\left(\nu_{2}\right)}, \ldots, x_{2}^{\left(\nu_{2}+n_{1}\right)}, z^{\left(\left\langle\nu, \gamma_{1}\right\rangle\right)}, z^{\left(\left\langle\nu, \gamma_{1}\right\rangle+1\right)}\right) .
$$


And in general, for $1 \leq k \leq r$

$$
\begin{aligned}
F_{1, \nu}^{\left(\frac{l_{1}(\nu)}{e_{1}}+k\right)} & =z^{\left(\left\langle\nu, \gamma_{1}\right\rangle\right)} h_{1,0}\left(z^{\left(\left\langle\nu, \gamma_{1}\right\rangle\right)}, \ldots, z^{\left(\left\langle\nu, \gamma_{1}\right\rangle+k\right)}\right)+z^{\left(\left\langle\nu, \gamma_{1}\right\rangle+1\right)} h_{1,1}\left(z^{\left(\left\langle\nu, \gamma_{1}\right\rangle+1\right)}, \ldots, z^{\left(\left\langle\nu, \gamma_{1}\right\rangle+k\right)}\right) \\
& +\cdots+z^{\left(\left\langle\nu, \gamma_{1}\right\rangle+\left[\frac{k}{n_{1}}\right]\right)} h_{1,\left[k / n_{1}\right]}\left(z^{\left(\left\langle\nu, \gamma_{1}\right\rangle+\left[\frac{k}{n_{1}}\right]\right)}, \ldots, z^{\left(\left\langle\nu, \gamma_{1}\right\rangle+k\right)}\right)+ \\
& +h_{2,0}\left(x_{1}^{\left(\nu_{1}\right)}, \ldots, x_{1}^{\left(\nu_{1}+k\right)}\right) x_{2}^{\left(\nu_{2}\right)}+h_{2,1}\left(x_{1}^{\left(\nu_{1}\right)}, \ldots, x_{1}^{\left(\nu_{1}+k\right)}\right) x_{2}^{\left(\nu_{2}+1\right)}+\cdots+ \\
& +h_{2, k}\left(x_{1}^{\left(\nu_{1}\right)}, \ldots, x_{1}^{\left(\nu_{1}+k\right)}\right) x_{2}^{\left(\nu_{2}+k\right)}
\end{aligned}
$$

where $h_{1,0}, h_{1,1}, \ldots, h_{1,\left[k / n_{1}\right]}, h_{2,0}, \ldots, h_{2, k}$ are polynomials. And since $k \leq r<\beta$ and $\left[\frac{m}{n}\right]=\left\langle\nu, \gamma_{1}\right\rangle+\alpha$ with $\alpha=\left[\frac{r}{n_{1}}\right] \geq\left[\frac{k}{n_{1}}\right]$, we have proved (25) as we wanted.

(ii) If $\nu^{\prime} \in L_{m}$, then by Lemma 4.18

$$
C_{m}^{\nu^{\prime}}=\overline{V\left(I^{\nu^{\prime}}, J_{m}^{\nu^{\prime}}\right) \cap D\left(x_{1}^{\left(\nu_{1}^{\prime}\right)}\right)} .
$$

Since $\nu \Varangle_{p} \nu^{\prime}$ we have that $I^{\nu} \subseteq I^{\nu^{\prime}}$. We are going to prove that the generators of $J_{m}^{\nu}$ modulo $\left(I^{\nu^{\prime}}, z^{(l)}\right)_{0 \leq l<\frac{l_{1}\left(\nu^{\prime}\right)}{n}}$ belong to $J_{m}^{\nu^{\prime}}$. Since $l_{i}(\nu)=l_{i}\left(\nu^{\prime}\right)$ for $1 \leq i \leq g_{1}$, we have that

$$
\begin{aligned}
& F_{i, \nu}^{\left(\frac{l_{i}(\nu)}{e_{i}}+r_{i}\right)}=F_{i, \nu^{\prime}}^{\left(\frac{l_{i}\left(\nu^{\prime}\right)}{e_{i}}+r_{i}\right)} \quad \text { for } 1 \leq i \leq g_{1}-1 \text { and } 0 \leq r_{i}<k_{i}(\nu)=k_{i}\left(\nu^{\prime}\right), \\
& F_{g_{1}, \nu}^{\left(\frac{l_{g_{1}}(\nu)}{g_{1}}+r\right)}=F_{g_{1}, \nu^{\prime}}^{\left(\frac{l_{g_{1}}\left(\nu^{\prime}\right)}{g_{1}}+r\right)} \quad \text { for } 0 \leq r<k_{g_{1}}(\nu)<k_{g_{1}}\left(\nu^{\prime}\right) .
\end{aligned}
$$

When $J_{m}^{\nu}$ has more generators, that is, when $j(m, \nu)>g_{1}$, then $j^{\prime}(m, \nu) \geq g_{1}$. We distinguish two cases.

(ii.a) If $j^{\prime}(m, \nu)=g_{1}$, then, by Corollary 4.15,

$$
J_{m}^{\nu}=\left(z^{(0)}, \ldots, z^{\left(\frac{l_{1}(\nu)}{n}-1\right)} ; F_{i, \nu}^{\left(\frac{l_{i}(\nu)}{e_{i}}+r_{i}\right)}, 1 \leq i \leq g_{1}, 0 \leq r_{i}<k_{i}(\nu) ; F_{g_{1}+1, \nu}^{\left(\frac{l_{g_{1}+1}(\nu)}{e_{11}+1}\right)}\right)_{l_{i}(\nu)<l_{i+1}(\nu)},
$$

and $l_{g_{1}+1}(\nu) \leq m<l_{g_{1}+1}(\nu)+e_{g_{1}+1}$. By (20) we have that

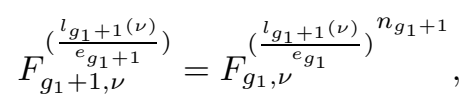

since $\nu_{2}^{\prime}>\nu_{2}$. Notice that $l_{g_{1}+1}(\nu)=l_{g_{1}}(\nu)+l_{g_{1}+1}(\nu)-l_{g_{1}}(\nu)=l_{g_{1}}\left(\nu^{\prime}\right)+\alpha \leq m$, with $\alpha>0$. Then $l_{g_{1}}\left(\nu^{\prime}\right)<m$ and therefore $j\left(m, \nu^{\prime}\right) \geq g_{1}$. If $j\left(m, \nu^{\prime}\right)>g_{1}$ then

$$
F_{g_{1}, \nu}^{\left(\frac{l_{g_{1}+1}(\nu)}{e_{g_{1}}}\right)} \in J_{m}^{\nu^{\prime}}
$$

since $\frac{l_{g_{1}+1}(\nu)}{e_{g_{1}}}=\frac{l_{g_{1}}\left(\nu^{\prime}\right)}{e_{g_{1}}}+k_{g_{1}}(\nu)$ and $k_{g_{1}}(\nu)<k_{g_{1}}\left(\nu^{\prime}\right)$. If $j\left(m, \nu^{\prime}\right)=g_{1}$ then

$$
F_{g_{1}, \nu}^{\left(\frac{l_{g_{1}+1}(\nu)}{e_{g_{1}}}\right)} \in J_{m}^{\nu^{\prime}}
$$

since $\frac{l_{g_{1}+1}(\nu)}{e_{g_{1}}}=\frac{l_{g_{1}}\left(\nu^{\prime}\right)}{e_{g_{1}}}+k_{g_{1}}(\nu)$, and $k_{g_{1}}(\nu) \leq\left[\frac{m-l_{g_{1}}\left(\nu^{\prime}\right)}{e_{g_{1}}}\right]$, because $l_{g_{1}}(\nu)=l_{g_{1}}\left(\nu^{\prime}\right)$ and $m \geq l_{g_{1}+1}(\nu)$.

(ii.b) If $j^{\prime}(m, \nu)>g_{1}$, then we are in the case $g_{2}=g_{1}+1$ and $j^{\prime}(m, \nu)=g_{1}+1$. There exists an integer $1 \leq r<k_{g_{1}+1}(\nu)$ such that

$$
l_{g_{1}+1}(\nu)+r e_{g_{1}+1} \leq m<l_{g_{1}+1}(\nu)+(r+1) e_{g_{1}+1} .
$$

Then $J_{m}^{\nu}=\left(z^{(0)}, \ldots, z^{\left(\frac{l_{1}(\nu)}{n}-1\right)}, F_{1, \nu}^{\left(\frac{l_{1}(\nu)}{e_{1}}\right)}, \ldots, F_{g_{1}, \nu}^{\left(\frac{l_{g_{1}}+k_{g_{1}}}{g_{1}}+(\nu)-1\right)}, F_{g_{1}+1, \nu}^{\left(\frac{l_{g_{1}+1}(\nu)}{e_{1}+1}\right)}, \ldots, F_{g_{1}+1, \nu}^{\left(\frac{l_{g_{1}+1}(\nu)}{e_{1}+1}+r\right)}\right)$, where

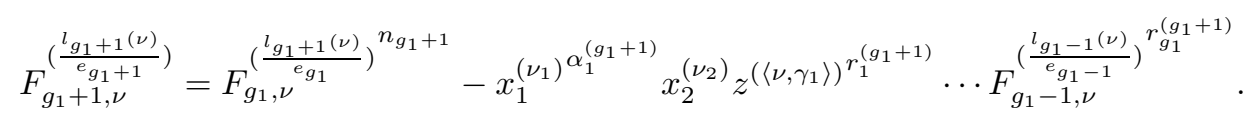




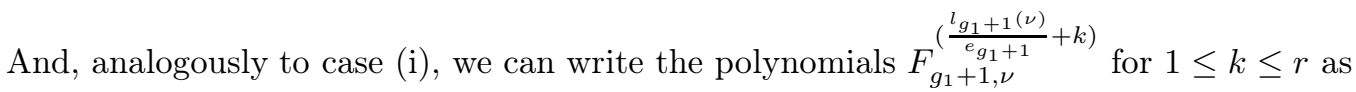

$$
\begin{aligned}
F_{g_{1}+1, \nu}^{\left(\frac{l_{g_{1}+1}(\nu)}{g_{1}+1}+k\right)} & =F_{g_{1}, \nu}^{\left(\frac{l_{g_{1}+1}(\nu)}{g_{1}}\right)} h_{1,0}+F_{g_{1}, \nu}^{\left(\frac{l_{g_{1}+1}(\nu)}{g_{g_{1}}}+1\right)} h_{1,1}+\cdots+F_{g_{1}, \nu}^{\left(\frac{l_{g_{1}+1}(\nu)}{g_{g_{1}}}+\left[\frac{k}{n_{g_{1}+1}}\right]\right)} h_{1,\left[k / n_{g_{1}+1}\right]}+ \\
& h_{2,0} x_{2}^{\left(\nu_{2}\right)}+\cdots+h_{2, k} x_{2}^{\left(\nu_{2}+k\right)},
\end{aligned}
$$

for certain polynomials $h_{1,0}, \ldots, h_{1,\left[k / n_{g_{1}+1}\right]}, h_{2,0}, \ldots, h_{2, k}$.

If $j\left(m, \nu^{\prime}\right)=g_{1}$ then $l_{g_{1}+1}\left(\nu^{\prime}\right)>m$ and by (26) it follows that $\beta>r$. Moreover

$$
J_{m}^{\nu^{\prime}}=\left(z^{(0)}, \ldots, z^{\left(\frac{l_{1}\left(\nu^{\prime}\right)}{n}-1\right)}, F_{1, \nu}^{\left(\frac{l_{1}\left(\nu^{\prime}\right)}{e}\right)}, \ldots, F_{g_{1}, \nu}^{\left(\frac{l_{g_{1}}\left(\nu^{\prime}\right)}{g_{1}}\right)}, \ldots, F_{g_{1}, \nu}^{\left(\frac{l_{g_{1}\left(\nu^{\prime}\right)}}{e_{1}}+\alpha\right)}\right)
$$

with $\alpha=\left[\frac{m-l_{g_{1}}\left(\nu^{\prime}\right)}{e_{g_{1}}}\right]$. We have that $\left[\frac{m-l_{g_{1}}\left(\nu^{\prime}\right)}{e_{g_{1}}}\right]=\left[\frac{m-l_{g_{1}}(\nu)}{e_{g_{1}}}\right]=\left[\frac{m}{e_{g_{1}}}\right]-\frac{l_{g_{1}}(\nu)}{e_{g_{1}}}$, since $\frac{l_{g_{1}}(\nu)}{e_{g_{1}}}$ is an integer, and by (26) we have that $\left\langle\nu, \gamma_{g_{1}+1}\right\rangle+\frac{r}{n_{g_{1}+1}} \leq \frac{m}{e_{g_{1}}}<\left\langle\nu, \gamma_{g_{1}+1}\right\rangle+\frac{r+1}{n_{g_{1}+1}}$. Then $\left[\frac{m}{e_{g_{1}}}\right]=$ $\left\langle\nu, \gamma_{g_{1}+1}\right\rangle+\left[\frac{r}{n_{g_{1}+1}}\right]$, and we have that

$$
\frac{l_{g_{1}+1}(\nu)}{e_{g_{1}}}+\left[\frac{k}{n_{g_{1}+1}}\right] \leq \frac{l_{g_{1}}(\nu)}{e_{g_{1}}}+\left[\frac{m-l_{g_{1}}(\nu)}{e_{g_{1}}}\right] .
$$

It follows that $F_{g_{1}+1, \nu}^{\left(\frac{l_{g_{1}+1}(\nu)}{e_{g_{1}+1}}+k\right)}$ belongs to $J_{m}^{\nu^{\prime}}$.

If $j\left(m, \nu^{\prime}\right)=g_{1}+1$ then $\nu^{\prime} \in N_{g_{+} 1}$ and therefore $\beta=q n_{g_{1}+1}$ with $q \in \mathbb{Z}_{>0}$. Moreover there exists an integer $0<r^{\prime}<k_{g_{1}+1}\left(\nu^{\prime}\right)$ such that

$$
l_{g_{1}+1}\left(\nu^{\prime}\right)+r^{\prime} e_{g_{1}+1} \leq m<l_{g_{1}+1}\left(\nu^{\prime}\right)+\left(r^{\prime}+1\right) e_{g_{1}+1} .
$$

Or equivalently $l_{g_{1}+1}(\nu)+\left(r^{\prime}+q n_{g_{1}+1}\right) e_{g_{1}+1} \leq m<l_{g_{1}+1}(\nu)+\left(r^{\prime}+q n_{g_{1}+1}+1\right) e_{g_{1}+1}$. Then by (26) it follows that

$$
r=r^{\prime}+q n_{g_{1}+1}
$$

and therefore $r>\beta$. Moreover $k_{g_{1}}\left(\nu^{\prime}\right)=k_{g_{1}}(\nu)+q n_{g_{1}+1}$. Then

$J_{m}^{\nu^{\prime}}=\left(z^{(0)}, \ldots, z^{\left(\frac{l_{1}\left(\nu^{\prime}\right)}{n}-1\right)}, F_{1, \nu}^{\left(\frac{l_{1}\left(\nu^{\prime}\right)}{e}\right)}, \ldots, F_{g_{1}, \nu}^{\left(\frac{l_{g_{1}}\left(\nu^{\prime}\right)}{e_{1}}+k_{g_{1}}\left(\nu^{\prime}\right)-1\right)}, F_{g_{1}+1, \nu}^{\left(\frac{l_{g_{1}+1}\left(\nu^{\prime}\right)}{e_{g_{1}+1}}\right)}, \ldots, F_{g_{1}+1, \nu}^{\left(\frac{l_{g_{1}+1}\left(\nu^{\prime}\right)}{e_{1}+1}+r^{\prime}\right)}\right)$,

where notice that $\frac{l_{g_{1}}\left(\nu^{\prime}\right)}{e_{g_{1}}}+k_{g_{1}}\left(\nu^{\prime}\right)-1=\frac{l_{g_{1}}(\nu)}{e_{g_{1}}}+k_{g_{1}}(\nu)+q n_{g_{1}+1}-1$, and

$$
J_{m}^{\nu}=\left(z^{(0)}, \ldots, z^{\left(\frac{l_{1}(\nu)}{n}-1\right)}, F_{1, \nu}^{\left(\frac{l_{1}(\nu)}{e}\right)}, \ldots, F_{g_{1}, \nu}^{\left(\frac{l_{g_{1}}(\nu)}{e_{1}}+k_{g_{1}}(\nu)-1\right)}, F_{g_{1}+1, \nu}^{\left(\frac{l_{g_{1}+1}(\nu)}{e_{g_{1}+1}}\right)}, \ldots, F_{g_{1}+1, \nu}^{\left(\frac{l_{g_{1}+1}(\nu)}{e_{1}+1}+r\right)}\right) .
$$

Since $I^{\nu^{\prime}}=\left(x_{1}^{(0)}, \ldots, x_{1}^{\left(\nu_{1}-1\right)}, x_{2}^{(0)}, \ldots, x_{2}^{\left(\nu_{2}+q n_{g_{1}+1}-1\right)}\right)$ it follows that for $0 \leq k \leq r^{\prime}$ and $s=q n_{g_{1}+1}+k$

$$
F_{g_{1}+1, \nu}^{\left(\frac{l_{g_{1}+1}(\nu)}{e_{1}+1}+s\right)}=F_{g_{1}+1, \nu}^{\left(\frac{l_{g_{1}+1}\left(\nu^{\prime}\right)}{e_{g_{1}+1}+1}+k\right)} .
$$

Then finally we have to prove that $F_{g_{1}+1, \nu} \frac{\left(\frac{l_{g_{1}+1}(\nu)}{e_{1}+1}+s\right)}{J_{m}} \in J_{m}^{\nu^{\prime}}$ for $0 \leq s<q n_{g_{1}+1}$. This follows as in the previous cases, since

$$
\begin{aligned}
F_{g_{1}+1, \nu}^{\left(\frac{l_{g_{1}+1}(\nu)}{e_{g_{1}+1}}\right)} & =F_{g_{1}, \nu}^{\left(\frac{l_{g_{1}+1}(\nu)}{e_{g_{1}}}\right)^{n_{g_{1}+1}}}-x_{1}^{\left(\nu_{1}\right)^{\alpha_{1}^{\left(g_{1}+1\right)}}} x_{2}^{\left(\nu_{2}\right)} z^{\left(\left\langle\nu, \gamma_{1}\right\rangle\right)^{r_{1}^{\left(g_{1}+1\right)}} \cdots} \\
& \text { and for } 0 \leq s<q n_{g_{1}+1} \\
F_{g_{1}+1, \nu}^{\left(\frac{l_{g_{1}+1}(\nu)}{e_{1}+1}+s\right)} & =F_{g_{1}, \nu}^{\left(\frac{l_{g_{1}+1}(\nu)}{g_{1}}\right)} h_{1,0}+\cdots+F_{g_{1}, \nu}^{\left(\frac{l_{g_{1}+1}(\nu)}{g_{1}}+\left[\frac{s}{n_{g_{1}+1}}\right]\right)} h_{1,\left[s / n_{g_{1}+1}\right]} \\
& -h_{2,0} x_{2}^{\left(\nu_{2}\right)}-\cdots-h_{2, s} x_{2}^{\left(\nu_{2}+s\right)}
\end{aligned}
$$




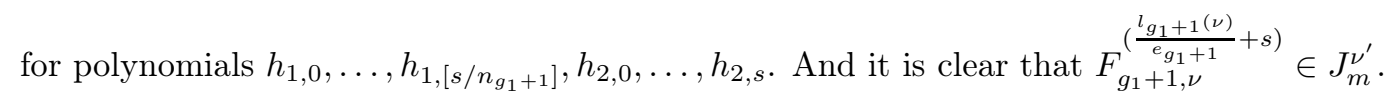

The key point in all the cases is that $\alpha_{2}^{\left(g_{1}+1\right)}=1$.

If $\nu^{\prime}-\nu \in \rho_{1}$ then we are in the case $\gamma_{1}=\left(\frac{1}{n_{1}}, \frac{1}{n_{1}}\right)$. Now $\nu_{1}^{\prime}>\nu_{1}$ and $\nu_{2}^{\prime}=\nu_{2}$, and similar arguments apply to this case to get the inclusion we want to prove.

The previous Proposition motivates the following definition.

Definition 4.22. We consider the relation in $N_{0}$, depending on $m$ and denoted by $<_{R, m}$, given by

$$
\nu \leq_{R, m} \nu^{\prime} \text { if and only if } \nu \leq_{p} \nu^{\prime} \text { and } \nu^{\prime}-\nu \in \sigma_{R e g, j^{\prime}(m, \nu)} .
$$

We define the set $F_{m}=\min _{\leq_{R, m}}\left\{P_{m} \cup L_{m}^{<}\right\}$.

Notice that, by (14), for $m$ and $\nu$ such that $j^{\prime}(m, \nu)>g_{2}$, this order is just equality.

It is worth pointing out that the inclusions which are described by this last relation in Proposition 4.21. can be explained by the fact that even though a curve may be in the singular locus of a quasi-ordinary surface, it may not be part of the singular locus of its first approximated quasi-ordinary surfaces. And as Proposition 4.13 explains, the geometry of $C_{m}^{\nu}$ is only determined by the geometry of one of its approximated roots, for $m$ small enough. Hence, the jets which project to the singular locus of the surface but not to the singular locus of the approximated surfaces will not give rise to irreducible components of the jet schemes for $m$ small enough, and they will be included in other components.

Now we prove that all possible inclusions are controlled by the relation defined in Definition 4.22 and the product ordering, that is, in the set $F_{m}$.

Proposition 4.23. Given $m \in \mathbb{Z}_{>0}$ and $\nu, \nu^{\prime} \in F_{m}$ with $\nu \leq_{p} \nu^{\prime}$ then $C_{m}^{\nu^{\prime}} \nsubseteq C_{m}^{\nu}$.

Proof. We will prove that $C_{m}^{\nu^{\prime}} \nsubseteq C_{m}^{\nu}$ by showing that

$$
\operatorname{Codim}\left(C_{m}^{\nu^{\prime}}\right) \leq \operatorname{Codim}\left(C_{m}^{\nu}\right)
$$

First notice that $\nu \in L_{m}^{<}$, since otherwise there would not exist $\nu^{\prime} \neq \nu$ such that $\nu^{\prime} \in F_{m}$ and $\nu \leq \nu_{p}^{\prime}$. Recall that $\sigma=\mathbb{R}_{\geq 0}^{2}$, we define the set

$$
\begin{aligned}
E(\nu)_{m} & =\left\{\nu^{\prime} \in(\nu+\sigma) \cap\left(P_{m} \cup L_{m}^{<}\right) \mid \nu^{\prime} \neq \nu \text { and it is minimal with respect to } \leq_{R, m} \text { in } \nu+\sigma\right\} \\
& =\left\{\nu^{\prime} \in(\nu+\sigma) \cap\left(P_{m} \cup L_{m}^{<}\right) \mid \nu^{\prime} \neq \nu \text { and } \nexists \widetilde{\nu} \in(\nu+\sigma) \cap\left(P_{m} \cup L_{m}^{<}\right) \text {such that } \widetilde{\nu} \leq_{R, m} \nu^{\prime}\right\} .
\end{aligned}
$$

We claim that for any $\nu^{\prime} \in E(\nu)_{m}$ we have that

$$
\operatorname{Codim}\left(C_{m_{0}}^{\nu^{\prime}}\right) \leq \operatorname{Codim}\left(C_{m_{0}}^{\nu}\right) \text { for } l_{1}(\nu)+e_{1} \leq m_{0}<l_{i(\nu)}(\nu) .
$$

We prove this claim by induction on $m$. For $m=l_{1}(\nu)+e_{1}$ we have that

(i) if $a_{1}=1$ then we are in the case $\gamma_{1}=\left(\frac{1}{n_{1}}, \frac{1}{n_{1}}\right)$ and $E(\nu)_{m}=\emptyset$, because $\nu+(1,0), \nu+(0,1) \notin N_{1}$ and $\nu+(2,0), \nu+(0,2) \in H_{m}$ but $\nu \leq_{R, m} \nu+(2,0)$ and $\nu \leq_{R, m} \nu+(0,2)$.

(ii) If $a_{1}>1$ then $\nu+(1,0) \in H_{m}$ and it follows that in fact $E(\nu)_{m}=\{\nu+(1,0)\}$, because the only other possible $\nu^{\prime}$ is

$$
\begin{array}{ll}
\nu^{\prime}=\nu+(0,1) \in P_{m} \cup L_{m} & \text { if } b_{1} \equiv 0 \bmod n_{1} \\
\nu^{\prime}=\nu+(0,2) \in P_{m} \cup L_{m} & \text { otherwise }
\end{array}
$$

and in both cases we have that $\nu \leq_{R, m} \nu^{\prime}$. Now, by Lemma 4.16 we have that for $\nu^{\prime}=\nu+(1,0)$,

$$
\operatorname{Codim}\left(C_{m}^{\nu^{\prime}}\right)=\nu_{1}+\nu_{2}+\frac{l_{1}(\nu)}{n}+2=\operatorname{Codim}\left(C_{m}^{\nu}\right)
$$

Suppose that the claim is true for $m-1$ and we prove it for $m$. Let $\nu^{\prime}$ be an element in $E(\nu)_{m}$.

(i) If $\nu^{\prime} \in E(\nu)_{m-1}$, by induction hypothesis, we have that $\operatorname{Codim}\left(C_{m-1}^{\nu^{\prime}}\right) \leq \operatorname{Codim}\left(C_{m-1}^{\nu}\right)$. By Corollary 4.17 we know that, passing from $m-1$ to $m$, the codimension of $C_{m}^{\nu}$ grows if and only if $m$ is divisible by $e_{j(m-1, \nu)}$, and it grows by one. But since $\nu \leq_{p} \nu^{\prime}$ we have that $j\left(m-1, \nu^{\prime}\right) \leq j(m-1, \nu)$ and therefore if $e_{j\left(m-1, \nu^{\prime}\right)}$ divides $m$ then $e_{j(m-1, \nu)}$ divides $m$, and it follows that $\operatorname{Codim}\left(C_{m}^{\nu^{\prime}}\right) \leq \operatorname{Codim}\left(C_{m}^{\nu}\right)$.

(ii) If $\nu^{\prime} \notin E(\nu)_{m-1}$, there exists $\widetilde{\nu} \in E(\nu)_{m-1}$ such that $\widetilde{\nu} \leq_{R, m-1} \nu^{\prime}$ and $\widetilde{\nu} \mathbb{E}_{R, m} \nu^{\prime}$. By induction hypothesis we have that $\operatorname{Codim}\left(C_{m-1}^{\widetilde{\nu}}\right) \leq \operatorname{Codim}\left(C_{m-1}^{\nu}\right)$, and again, since $\nu \lesseqgtr \widetilde{\nu}$ then $j(m, \nu) \geq j(m, \widetilde{\nu})$ and 
JET SCHEMES OF QUASI-ORDINARY SURFACE SINGULARITIES

therefore $\operatorname{Codim}\left(C_{m}^{\widetilde{\nu}}\right) \leq \operatorname{Codim}\left(C_{m}^{\nu}\right)$. Now we are going to prove that $\operatorname{Codim}\left(C_{m}^{\nu^{\prime}}\right) \leq \operatorname{Codim}\left(C_{m}^{\widetilde{\nu}}\right)$. We have two possibilities, either $\widetilde{\nu} \in L_{m}^{<}$or $\widetilde{\nu} \notin L_{m}^{<}$.

If $\widetilde{\nu} \in L_{m}^{<}$, then $m=l_{g_{2}+1}(\widetilde{\nu})+e_{g_{2}+1}$ and

$$
\pi_{m, m-1}^{-1}\left(O_{m-1}^{\widetilde{\nu}}\right)=V\left(I^{\widetilde{\nu}}, z^{(0)}, \ldots, z^{\left(\left(\widetilde{\nu}, \gamma_{1}\right\rangle-1\right)}, F_{1, \widetilde{\nu}}^{\left(\frac{l_{1}(\widetilde{\nu})}{e_{1}}\right)}, \ldots, F_{g_{2}+1, \widetilde{\nu}}^{\left(\frac{l_{g_{2}+1}(\widetilde{\nu})}{e_{g_{2}}+1}\right)}, F_{g_{2}+1, \widetilde{\nu}}^{\left(\frac{l_{g_{2}+1}(\widetilde{\nu})}{e_{g_{2}+1}}+1\right)}\right) \cap D\left(x_{1}^{(\widetilde{\nu})}\right),
$$

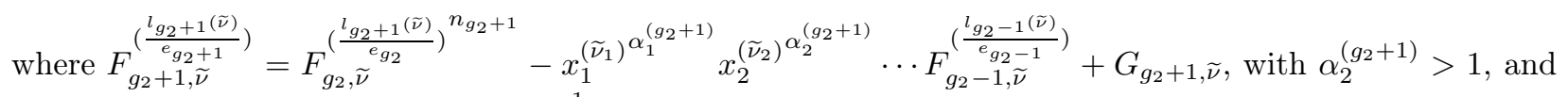
$F_{g_{2}+1, \widetilde{\nu}}^{\left(\frac{l_{g_{2}+1}(\widetilde{\nu})}{e_{g_{2}+1}}+1\right)}=n_{g_{2}+1} F_{\left.g_{2}, \frac{\left(g_{2}\right.}{\left(g_{2}\right.}\right)^{l_{2}}} F_{g_{2}, \widetilde{\nu}}^{\left(\frac{l_{g_{2}+1}(\widetilde{\nu})}{g_{2}}+1\right)}-x_{2}^{(\widetilde{\nu})} H$, where $H$ is a polynomial in the variables $H\left(x_{1}^{\left(\widetilde{\nu}_{1}\right)}, x_{1}^{\left(\widetilde{\nu}_{1}+1\right)}, x_{2}^{\left(\widetilde{\nu}_{2}\right)}, x_{2}^{\left(\widetilde{\nu}_{2}+1\right)}, \ldots, F_{g_{2}-1, \widetilde{\nu}}^{\left(\frac{l_{g_{2}-1}(\widetilde{\nu})}{e_{g_{2}}-1}\right)}, F_{g_{2}-1, \widetilde{\nu}}^{\left(\frac{l_{g_{2}-1}(\widetilde{\nu})}{e_{g_{2}-1}}+1\right)}\right)$. Then

$$
\left(\pi_{m, m-1}^{-1}\left(C_{m-1}^{\widetilde{\widetilde{\nu}}}\right)\right)_{r e d}=\overline{V\left(I^{\widetilde{\nu}}, J_{m}^{\widetilde{\nu}}\right) \cap D\left(x_{1}^{\left(\widetilde{\nu}_{1}\right)}\right) \cap V\left(x_{2}^{\left(\widetilde{\nu}_{2}\right)}\right)} \cup \overline{V\left(I^{\widetilde{\nu}}, J_{m}^{\widetilde{\nu}}\right) \cap D\left(x_{1}^{\left(\widetilde{\nu}_{1}\right)}\right) \cap D\left(x_{2}^{\left(\widetilde{\nu}_{2}\right)}\right)}
$$

and it is not difficult to see that $\left(\pi_{m, m-1}^{-1}\left(C_{m-1}^{\widetilde{\nu}}\right)\right)_{r e d}=C_{m}^{\nu^{\prime}} \cup C_{m}^{\widetilde{\nu}}$, where $\nu^{\prime}=\widetilde{\nu}+(0, \alpha)$, with

$$
\alpha=\left\{\begin{array}{cc}
1 & \text { if } g_{2}=g_{1} \\
\min \left\{n_{g_{1}+1}, k_{g_{1}+1}(\widetilde{\nu})\right\} & \text { otherwise }
\end{array}\right.
$$

where remember that $k_{i}(\widetilde{\nu})$ denotes $\frac{l_{i+1}(\widetilde{\nu})-l_{i}(\widetilde{\nu})}{e_{i}}$. In both cases we have, by Proposition 4.16, that $\operatorname{Codim}\left(C_{m}^{\nu^{\prime}}\right)=$ $\operatorname{Codim}\left(C_{m-1}^{\widetilde{\nu}}\right)+1=\operatorname{Codim}\left(C_{m}^{\widetilde{\nu}}\right)$.

If $\widetilde{\nu} \notin L_{m}^{<}$then $m=l_{i(\widetilde{\nu})}(\widetilde{\nu})$ with $i(\widetilde{\nu}) \leq g_{2}+1$, since $j^{\prime}(m-1, \widetilde{\nu}) \leq g_{2}$. We have that $\left(\pi_{m, m-1}^{-1}\left(O_{m-1}^{\widetilde{\nu}}\right)\right)_{\text {red }}=$

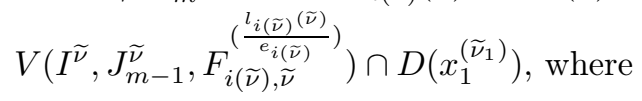

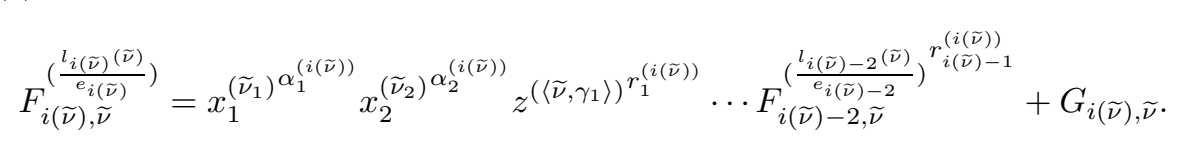

Therefore, by Lemma 5.4, $F_{i(\widetilde{\nu}), \widetilde{\nu}}^{\left(\frac{\left.l_{i(\widetilde{\nu})(\widetilde{\nu})}\right)}{e^{(}}\right)}=0$ implies that $x_{2}^{\left(\widetilde{\nu}_{2}\right)}=0$ because $i(\widetilde{\nu})-2<g_{2}$. And, as before, if $g_{2}=g_{1}+1$ and $i(\widetilde{\nu})=g_{2}+1$ then we have that $\nu^{\prime}=\widetilde{\nu}+(0, \alpha)$ with $\alpha=\min \left\{n_{g_{1}+1}, k_{g_{1}+1}(\widetilde{\nu})\right\}$. Otherwise $\nu^{\prime}=\widetilde{\nu}+(0,1)$, and in both cases we have

$$
\left(\pi_{m, m-1}^{-1}\left(C_{m-1}^{\widetilde{\nu}}\right)\right)_{r e d}=C_{m}^{\nu^{\prime}}
$$

with $\operatorname{Codim}\left(C_{m}^{\nu^{\prime}}\right)=\operatorname{Codim}\left(C_{m-1}^{\widetilde{\nu}}\right)+1$. Since $\widetilde{\nu} \in E(\nu)_{m-1}$, it follows that $j(m-1, \nu)>j(m-1, \widetilde{\nu})=i(\widetilde{\nu})-1$ and by Corollary 4.17 we have that $\operatorname{Codim}\left(C_{m}^{\nu}\right)=\operatorname{Codim}\left(C_{m-1}^{\nu}\right)+1$, which finishes the proof.

Now we can prove the main theorem of this section.

Theorem 4.24. For $m \in \mathbb{Z}_{>0}$ the decomposition of $\pi_{m}^{-1}\left(X_{\text {Sing }}\right)$ in irreducible components is given by

$$
\left(\pi_{m}^{-1}\left(X_{\text {Sing }}\right)\right)_{r e d}=\bigcup_{\nu \in F_{m}} C_{m}^{\nu}
$$

Proof. The irreducibility of the sets $C_{m}^{\nu}$ was proven in Proposition 4.16. And by Proposition 4.19, Proposition 4.21 and Proposition 4.23 we have that

$$
\bigcup_{\nu \in H_{m} \cup L_{m}} C_{m}^{\nu}=\bigcup_{\nu \in F_{m}} C_{m}^{\nu}
$$

Hence the result follows by Lemma 4.10 .

Remark 4.25. For $\nu \in N_{g}$, if $\nu \in F_{l_{g}(\nu)}$, then $\nu \in F_{m}$ for every $m \geq l_{g}(\nu)$, or in other words, $\nu$ gives rise to an irreducible component for any $m \geq l_{g}(\nu)$. 
Remark 4.26. When the equisingular dimension is $c=1$ (see Definition 3.14), then $g_{1}=g_{2}=g$. Moreover we have the following properties for $1 \leq i \leq g$

$$
\begin{aligned}
& l_{i}(\nu)=l_{i}(\nu+(0, r)), \text { for all } r \in \mathbb{Z} \\
& \text { if } \nu \in N_{i} \text { then } \nu+(0, r) \in N_{i}, \text { for all } r \in \mathbb{Z}
\end{aligned}
$$

Hence we deduce that for any $m \in \mathbb{Z}_{>0}$ and $\nu \in H_{m} \cup L_{m}$ we have $\sigma_{\text {Reg, } j^{\prime}(m, \nu)}=\rho_{2}$, and therefore $F_{m}=$ $\left(P_{m} \cup L_{m}^{<}\right) \cap \rho_{1}$.

The behaviour of the jet schemes is exactly as the plane curve defined by the Puiseux pairs $\lambda_{1}^{(1)}, \ldots, \lambda_{g}^{(1)}$. In [26] the second author describes the irreducible components of jets through the origin in the case of plane curves .

The previous remark is the simplest evidence of the fact that the irreducible components are only affected by the topological type. This is proved in Theorem 4.29

To any quasi-ordinary surface singularity we can associate a weighted graph, containing information about the irreducible components of jet schemes and how they behave under truncation maps.

Definition 4.27. The weighted graph of the jet schemes of $X$ is the leveled weighted graph $\Gamma$ defined as follows:

- for $m \geq 1$ we represent every irreducible components of $\pi_{m}^{-1}\left(X_{\text {Sing }}\right)$ by a vertex $V_{m}$, the sub-index $m$ being the level of the vertex;

- we join the vertices $V_{m+1}$ and $V_{m}$ if the canonical morphism $\pi_{m+1, m}$ induces a morphism between the corresponding irreducible components;

- we weight each vertex by the dimension of the corresponding irreducible component.

We define $E \Gamma$ to be the weighted graph that we obtain from $\Gamma$ by weighting any vertex of $\Gamma$ by the embedding dimensions of the corresponding irreducible components (note that by the definition of $\Gamma$, these vertices are also weighted by their dimensions).

Notice that the data of the codimension together with the embedded dimension permits to distinguish when the vertex corresponds to a hyperplane or a lattice component. Indeed, given a vertex of the graph, let $e$ be the embedded dimension and $c$ the codimension, then the vertex corresponds to a hyperplane component if and only if $e+c=3(m+1)$. Therefore we can extract from $E \Gamma$ a subgraph $\Gamma^{\prime}$ as follows.

Definition 4.28. We define a weighted subgraph $\Gamma^{\prime}$ of $E \Gamma$ by adding the condition that we join the vertices $V_{m}$ (corresponding to a certain component, say $C_{m}^{\nu^{\prime}}$ ) and $V_{m-1}$ (corresponding to $C_{m}^{\nu}$ ) only if

- if $\nu \in L_{m-1}^{<}$with $j(m-1, \nu) \leq g_{2}$ then $\nu^{\prime}=\nu+(0, \alpha)$ with $\alpha$ minimal among the elements in $F_{m}$.

- if $\nu \in L_{m-1}^{<}$with $j(m-1, \nu)>g_{2}$ then $\nu^{\prime}=\nu$.

The important thing about this new graph $\Gamma^{\prime}$ is that, with the weights, we are able to detect when we pass from a hyperplane component at level $m$ to a lattice component at level $m+1$, as we also do in the graph $E \Gamma$, but now we can follow this component in a unique path in the graph as $m$ grows. This will be useful to prove the following result.

Theorem 4.29. The graph $\Gamma^{\prime}$ determines and it is determined by the topological type of the singularity.

Proof. Obviously the graph is determined by the semigroup, and therefore, by [16, by the topological type.

To prove the converse we consider two different sets of generators of the semigroup $\left\{\gamma_{1}, \ldots, \gamma_{g}\right\}$ and $\left\{\gamma_{1}^{\prime}, \ldots, \gamma_{g^{\prime}}^{\prime}\right\}$ and we will prove that the corresponding weighted graphs are different too.

Given a weighted graph we can recover the number of characteristic exponents in the following way. Any vertex $V_{m}$ on the graph comes with the codimension $c\left(V_{m}\right)$ and the embedded dimension $e\left(V_{m}\right)$. Take an infinite branch, and consider the finite part that starts at

$$
m_{0}=\max \left\{m \mid V_{m-1} \text { is a hyperplane component and } V_{m} \text { is a lattice component }\right\},
$$

and ends at

$$
m_{1}=\min \left\{m \mid c\left(V_{m}\right)=c\left(V_{m-1}\right)+1 \text { for all } m>m_{1}\right\} .
$$

We can read $e_{0}, \ldots, e_{g-1}$ making use of Corollary 4.17. Indeed, along the piece of branch, the vertex $V_{m}$ corresponds to a component $C_{m}^{\nu}$ with $\nu \in N_{g}, m_{0}=l_{1}(\nu)$ and $m_{1}=l_{g}(\nu)$. To read this data we consider 
only branches that projects into the component $Z_{1}$ of the singular locus, since otherwise we can only assure that $m_{0}=l_{m(\nu)}(\nu)$, and we do not have all the information whenever $m(\nu)>1$. Notice that $Z_{1}$ is always a component of the singular locus unless we are in the case $g=1$ and $\gamma=\left(\frac{1}{n}, \frac{1}{n}\right)$, which is very easy to recognize. Indeed, it is the only case when at level $m=1$ we have only one component, with codimension 3 and embedded dimension 0. Moreover the multiplicity $n$ equals the first time $m$ when we have a lattice component. Therefore this simple case is very easily understood in the graph. For the rest of the cases, since we know that $\nu \in N_{g}$, going backwards we look for the biggest $m^{\prime}$ such that $c\left(V_{m^{\prime}}\right)=c\left(V_{m_{0}}\right)-1$. Then $n=m_{0}-m^{\prime}$. Now, going from level $m_{0}$ to $m_{1}$, we know that the codimension grows by one exactly every $e_{1}$ steps at first, after every $e_{2}$ steps, and so on. Since $e_{1}>e_{2}>\cdots>e_{g}=1$ we can read these numbers on the graph. Notice that equivalently we get $n_{1}, \ldots, n_{g}$, and in particular we have $g$, the number of characteristic exponents.

Suppose now that the number of generators of the semigroups is the same, say $g$. We will prove by induction on $g$ that the graphs corresponding to different sets of generators, are different. We denote the vertices at level $m$ by $V_{m}\left(c\left(V_{m}\right), e\left(V_{m}\right)\right)$. For $g=1$, the multiplicity is read from the graph as was explained before, and the situation for $m=1$ is:

$$
\begin{array}{cl}
\bullet V_{1}(3,0) & \text { if } \gamma=\left(\frac{1}{n}, \frac{1}{n}\right) \\
\bullet V_{1}(2,1) & \text { if } \gamma=\left(\frac{a}{n}, \frac{1}{n}\right), \text { with } a>1 \\
\bullet V_{1}(2,1) \bullet V_{1}(2,1) & \text { if } \gamma=\left(\frac{a}{n}, \frac{b}{n}\right), \text { with } b>1
\end{array}
$$

If we want to compare the graph associated to $\gamma$ and the graph associated to $\gamma^{\prime}$, we just have to consider the cases $\gamma=\left(\frac{a}{n}, \frac{1}{n}\right), \gamma^{\prime}=\left(\frac{a^{\prime}}{n}, \frac{1}{n}\right)$ with $a \neq a^{\prime}$, and $\gamma=\left(\frac{a}{n}, \frac{b}{n}\right), \gamma^{\prime}=\left(\frac{a^{\prime}}{n}, \frac{b^{\prime}}{n}\right)$ with $b, b^{\prime} \neq 1$ and $\gamma \neq \gamma^{\prime}$. The first case is very easy to distinguish, since the first moment a component splits in two is at $m=a$ for one graph, and at $m=a^{\prime}$ for the other. For the other case, first note that the graph of any quasi-ordinary with only one characteristic exponent $\gamma=\left(\frac{a}{n}, \frac{b}{n}\right)$ is the graph associated to $z^{n}-x_{1}^{a} x_{2}^{b}$. The key point is that, when $b>1$ we have in the graph a branch which generically corresponds to $x_{2}^{(0)} \neq 0$ (resp. $x_{1}^{(0)} \neq 0$ ), that is, it behaves like the graph of the curve $z^{n}-x_{1}^{a}$ (resp. $z^{n}-x_{2}^{b}$ ). Therefore comparing graphs associated to $\gamma$ and $\gamma^{\prime}$ with $\gamma \neq \gamma^{\prime}$, we deduce from Theorem 3.3 in [26], that the graphs must be different.

Now, suppose it is true for $g-1$ characteristic exponents, and we will prove it for $g$. From Proposition 4.13 we deduce that is sufficient to prove that the graphs associated to the sets $\left\{\gamma_{1}, \ldots, \gamma_{g-1}, \gamma_{g}\right\}$ and $\left\{\gamma_{1}, \ldots, \gamma_{g-1}, \gamma_{g}^{\prime}\right\}$ are different, since otherwise it holds by induction hypothesis. Moreover, since we read the integers $n_{1}, \ldots, n_{g}$ in the graph, we assume that $n_{g}^{\prime}=n_{g}$. As in the case $g=1$, by looking at the singular locus (which is seen at $m=1$ ) we just have to consider the case $\gamma_{g}^{(2)}=\gamma_{g}^{\prime(2)}=\frac{1}{n_{g}}$ and the case $\gamma_{g}^{(2)}, \gamma_{g}^{\prime(2)}>\frac{1}{n_{g}}$. In the first case $\gamma_{g}^{(1)} \neq \gamma_{g}^{\prime(1)}$ and $\gamma_{i}^{(2)}=\gamma_{i}^{\prime(2)}=0$ for $1 \leq i \leq g-1$. Therefore the graphs are the same till we get to level $m=\min \left\{n_{g}\left\langle\nu, \gamma_{g}\right\rangle, n_{g}\left\langle\nu, \gamma_{g}^{\prime}\right\rangle\right\}$, where $\nu=\left(\nu_{1}, 0\right) \in \sigma_{\text {Sing }} \cap N_{g-1}$ with $\nu_{1}$ smallest with this property. Since $\left\langle\nu, \gamma_{g}\right\rangle \neq\left\langle\nu, \gamma_{g}^{\prime}\right\rangle$ the graphs must differ at some moment. Finally, when $\gamma \neq \gamma^{\prime}$ with $\gamma_{g}^{(2)}, \gamma_{g}^{\prime(2)}>\frac{1}{n_{g}}$, again by Proposition 4.13, the graphs must be the same for $\left\{\gamma_{1}, \ldots, \gamma_{g}\right\}$ and $\left\{\gamma_{1}, \ldots, \gamma_{g-1}, \gamma_{g}^{\prime}\right\}$, till the last approximated root, that is, $f$, starts playing a role in the definition of a component, say $C^{\nu}$. Since $\left\langle\nu, \gamma_{g}\right\rangle \neq\left\langle\nu, \gamma_{g}^{\prime}\right\rangle$ we will see the difference on the graphs at level $m=\min \left\{n_{g}\left\langle\nu, \gamma_{g}\right\rangle, n_{g}\left\langle\nu, \gamma_{g}^{\prime}\right\rangle\right\}$.

4.3. Log-canonical threshold. In [32], Mustaţa gave a formula of the log-canonical threshold in terms of the codimension of jet schemes, which in our setting can be stated as

$$
l c t(f)=\min _{m \geq 0} \frac{\operatorname{Codim}\left(X_{m}\right)}{m+1} .
$$

Then, as an application to Theorem 4.24] we can recover, for the case of surfaces, the result in $[8]$.

Corollary 4.30. The log-canonical threshold of a quasi-ordinary surface singularity is given by: 


$$
l c t_{0}\left(X, \mathbb{A}^{3}\right)=\left\{\begin{array}{cl}
\frac{1+\lambda_{1}^{(1)}}{e_{0} \lambda_{1}^{(1)}} & \text { if } \lambda_{1} \neq\left(\frac{1}{n_{1}}, \frac{1}{n_{1}}\right) \\
1 & \text { if } \lambda_{1}=\left(\frac{1}{n_{1}}, \frac{1}{n_{1}}\right) \text { and } g=1 \\
\frac{n_{1}\left(1+\lambda_{2}^{(1)}\right)}{e_{1}\left(n_{1}\left(1+\lambda_{2}^{(1)}\right)-1\right)} & \text { if } \lambda_{1}=\left(\frac{1}{n_{1}}, \frac{1}{n_{1}}\right) \text { and } g>1
\end{array}\right.
$$

Moreover, the components that contribute to the log canonical threshold are

$$
\begin{aligned}
& C_{l_{1}(\nu)-1}^{\nu} \quad \text { if } \gamma_{1} \neq\left(\frac{1}{n_{1}}, \frac{1}{n_{1}}\right) \text { or } g=1 \\
& C_{l_{2}(\nu)-1}^{\nu} \quad \text { otherwise }
\end{aligned}
$$

where $\nu=(l, 0) \in N_{1}$ if $\gamma_{1} \neq\left(\frac{1}{n_{1}}, \frac{1}{n_{1}}\right)$ and $\nu=(l, 0) \in N_{2}$ otherwise.

Proof. The case $\lambda_{1}=\left(\frac{1}{n_{1}}, \frac{1}{n_{1}}\right)$ and $g=1$ behaves as an $A_{n}$-singularity, and then $l c t(f)=1$. For the rest of the cases, by Corollary 4.17, the codimension of a component grows faster as $m$ grows, for bigger $j(m, \nu)$. Therefore, the smaller codimension will be attached for $\nu \in P_{m} \cap F_{m}$, and more concretely for $\nu \in H_{m} \cap F_{m}$ whenever $H_{m} \cap F_{m} \neq \emptyset$. If $g_{1}=0$, since $a_{1} \geq b_{1}$, we deduce that the minimal codimension among the elements in $P_{m} \cap F_{m}$ is attached for $\nu$ of the form $\nu=(l, 0)$, while if $g_{1}>0$ then $P_{m} \cap F_{m}$ consists of just a point of the form $\nu=(l, 0)$.

We want to minimize not just the codimension, but the quotient $\frac{\operatorname{Codim}\left(X_{m}\right)}{m+1}$. That is, to find the biggest $m$ such that $\nu$ still belongs to $P_{m} \cap F_{m}$. Then, when the first characteristic exponent is different from $\left(\frac{1}{n_{1}}, \frac{1}{n_{1}}\right)$, this is attached for $m=l_{1}(\nu)-1$ such that $\nu \in L_{m+1}^{=}$. Then $m=l_{1}(l, 0)-1$ and $\operatorname{Codim}\left(C_{m}^{\nu}\right)=l+\left[\frac{m}{n}\right]+1$, and since $\nu \in L_{m+1}^{=},(l, 0) \in N_{1}$ and therefore $\operatorname{Codim}\left(C_{m}^{\nu}\right)=l+l \frac{a_{1}}{n_{1}}$, which implies that $\frac{\operatorname{Codim}\left(C_{m}^{\nu}\right)}{m+1}=\frac{a_{1}+n_{1}}{n a_{1}}$.

If $\gamma_{1}=\left(\frac{1}{n_{1}}, \frac{1}{n_{1}}\right)$ and $g>1$, what happens is that when $m=l_{1}(\nu)$ there is no subdivision of the component and $\sigma_{R e g, 1}=\rho_{1} \cup \rho_{2}$. If we denote the second exponent by $\gamma_{2}=\left(\frac{\alpha_{2}}{n_{1} n_{2}}, \frac{\beta_{2}}{n_{1} n_{2}}\right)$, we look for $\nu$ of the form $(l, 0)$ such that $m+1=l_{2}(\nu)$ with $\nu \in N_{2}$. Then $\operatorname{Codim}\left(C_{m}^{\nu}\right)=l+\frac{l_{1}(\nu)}{n}+\left[\frac{m-l_{1}(\nu)}{e_{1}}\right]+1=l+\frac{l_{1}(\nu)}{n}+\frac{l_{2}(\nu)-l_{1}(\nu)}{e_{1}}$, and therefore $\frac{\operatorname{Codim}\left(C_{m}^{\nu}\right)}{m+1}=\frac{l+l \frac{1}{n_{1}}+\frac{1}{e_{1}}\left(e_{2} n_{2} l \frac{\alpha_{2}}{n_{1} n_{2}}-e_{1} n_{1} l \frac{1}{n_{1}}\right)}{e_{2} n_{2} l \frac{\alpha_{2}}{n_{1} n_{2}}}=\frac{1+\frac{1}{n_{1}}+\frac{\alpha_{2}}{n_{1} n_{2}}-1}{e_{2} \frac{\alpha_{2}}{n_{1}}}=\frac{1+\frac{\alpha_{2}}{n_{2}}}{e_{1} \frac{\alpha_{2}}{n_{2}}}$. This coincides with the statement since $\lambda_{2}=\left(\frac{\alpha_{2}}{n_{1} n_{2}}-\frac{n_{1}-1}{n_{1}}, \frac{\beta_{2}}{n_{1} n_{2}}-\frac{n_{1}-1}{n_{1}}\right)$.

Remark 4.31. Notice that $\frac{1+\lambda_{1}^{(1)}}{e_{0} \lambda_{1}^{(1)}} \leq 1$ except in the case $\lambda_{1}=\left(\frac{1}{n_{1}}, \frac{1}{n_{1}}\right)$ and $g=1$. Moreover in the case of surfaces the condition $\lambda_{1}^{(1)}=\frac{1}{n_{1}}$ is equivalent to $\lambda_{1}=\left(\frac{1}{n_{1}}, \frac{1}{n_{1}}\right)$ since the branch is normalized. Then, see notations in [8], $\ell_{1}=\ell_{2}$ and in Theorem 4.30 we recover, for the case of surfaces, the formula given in Theorem 3.1 in 8 .

We now deduce a family of examples whose log canonical threshold can not be computed by a monomial valuation.

Corollary 4.32. Let $X$ be a quasi-ordinary surface singularity with $g>1$ characteristic exponents, and such that $\lambda_{1}=\left(\frac{1}{n_{1}}, \frac{1}{n_{1}}\right)$. Then lct $\left(X, \mathbb{A}^{3}\right)$ can not be contributed by monomial valuations in any variables.

Proof. It follows from Corollary 4.30 that $l c t\left(X, \mathbb{A}^{3}\right)$ is contributed by $C_{l_{1}(\nu)}^{\nu}$, for $\nu$ as is made precise in the above statement. This is equivalent to say that the valuation

$$
\begin{aligned}
\mathcal{V}_{C_{l_{2}(\nu)-1}^{\nu}}: \mathbb{C}\left[\left[x_{1}, x_{2}, z\right]\right] & \longrightarrow \mathbb{N} \\
h & \longmapsto \operatorname{ord}_{t}(h \circ \eta)
\end{aligned}
$$

where $\eta$ is the generic point of $\left(\Psi_{l_{2}(\nu)-1}^{\mathbb{A}^{3}}\right)^{-1}\left(C_{l_{2}(\nu)-1}^{\nu}\right)$. Note that $\nu$ can take all the values described in Corollary 4.30 but since $z^{\left(\left\langle\nu, \gamma_{1}\right\rangle\right)^{n_{1}}}-x_{1}^{\left(\nu_{1}\right)} x_{2}^{\left(\nu_{2}\right)}=0$ is one of the defining equations of $C_{l_{2}(\nu)-1}^{\nu}$, then $\mathcal{V}_{C_{l_{2}(\nu)-1}^{\nu}}\left(z^{n_{1}}-x_{1} x_{2}\right)>n_{1} \mathcal{V}_{C_{l_{2}(\nu)-1}^{\nu}}(z)$ and $\mathcal{V}_{C_{l_{2}(\nu)-1}^{\nu}}\left(z^{n_{1}}-x_{1} x_{2}\right)>\mathcal{V}_{C_{l_{2}(\nu)-1}^{\nu}}\left(x_{1}\right)+\mathcal{V}_{C_{l_{2}(\nu)-1}^{\nu}}\left(x_{2}\right)$. Therefore $\mathcal{V}_{l_{l_{2}(\nu)-1}^{\nu}}$ is not a monomial valuation. 
4.4. Example. Consider the quasi-ordinary surface $f=\left(\left(z^{2}-x_{1}^{3}\right)^{2}-x_{1}^{7} x_{2}^{3}\right)^{2}-x_{1}^{11} x_{2}^{5}\left(z^{2}-x_{1}^{3}\right)$. The semigroup is generated by the vectors

$$
\gamma_{1}=\left(\frac{3}{2}, 0\right), \gamma_{2}=\left(\frac{7}{2}, \frac{3}{2}\right) \text { and } \gamma_{3}=\left(\frac{29}{4}, \frac{13}{4}\right) .
$$

We have that $g_{1}=g_{2}=1$. The singular locus is reducible, of the form

$$
X_{\text {Sing }}=\left\{z=x_{1}=0\right\} \cup\left\{x_{2}=z^{2}-x_{1}^{3}=0\right\}=Z_{1} \cup Z_{2} .
$$

Then $\sigma_{\text {Sing }}=\mathbb{R}_{>0}^{2} \backslash\{0\}$ and $\sigma_{\text {Reg, }, 1}=\rho_{2}, \sigma_{\text {Reg }, 2}=\sigma_{\text {Reg, } 3}=\{(0,0)\}$.

The set $F_{m}$ describing the irreducible components is the following, for some $m$ :

$$
\begin{aligned}
& F_{m}=\{(1,0),(0,1)\}, \text { for } 1 \leq m<6 \\
& F_{m}=\{(1,0),(0,2)\}, \text { for } 6 \leq m<12 \\
& F_{12}=\{(2,0),(0,2)\} \\
& F_{13}=\{(2,0),(0,3)\} \\
& F_{18}=\{(2,0),(0,4)\} \\
& F_{26}=\{(2,0),(0,4),(0,5)\} \\
& F_{28}=\{(3,0),(2,0),(0,4),(0,5)\}
\end{aligned}
$$

and the result can be checked by lifting the components $Z_{1}$ and $Z_{2}$ of the singular locus to level $m$ as the following graph shows (we did not draw the weights of the vertices for clearness).

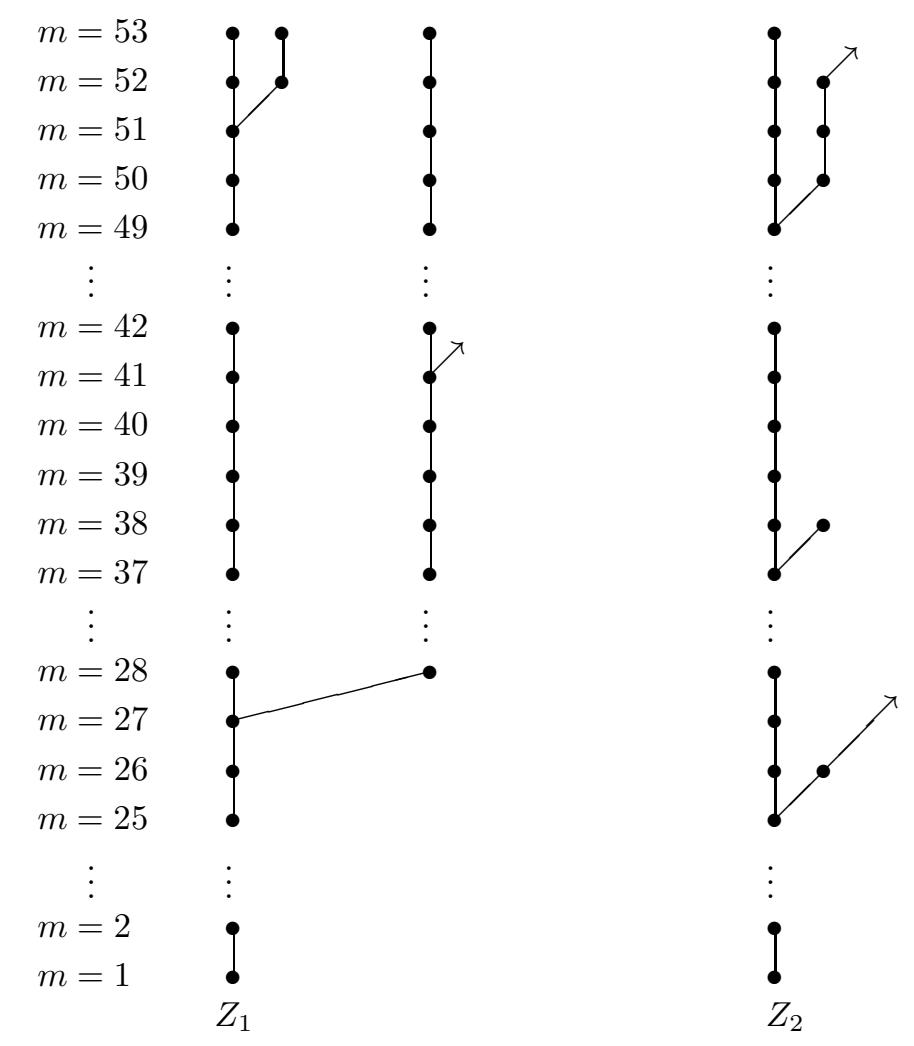

Figure 1 . The graph of the surface defined by $f=\left(\left(z^{2}-x_{1}^{3}\right)^{2}-x_{1}^{7} x_{2}^{3}\right)^{2}-x_{1}^{11} x_{2}^{5}\left(z^{2}-x_{1}^{3}\right)$.

The arrows in the figure represent the behaviour explained in Remark 4.25 , 
Now we give some explanations to illustrate how Proposition 4.19 and Proposition 4.21 work.

For $m=1$, we have $H_{1}=\{(1,0),(0,1)\}, L_{1}^{=}\{(0,1)\}$ and $L_{1}^{<}=\emptyset$. The claim on $F_{1}$ in this case follows easily by Proposition 4.19,

At level $m=6$ we have $H_{6}=\left\{\nu \in[0, m]^{2} \cap N_{0} \mid \nu_{1} \neq 0\right\}, L_{6}^{=}=\emptyset$ and $L_{6}^{<}=\left\{\left(0, \nu_{2}\right) \mid 2 \leq \nu_{2} \leq 6\right\}$ with $j^{\prime}(6,(0,2))=1$, then, by Proposition $4.21 C_{6}^{\nu^{\prime}} \subseteq C_{6}^{(0,2)}$ for $\nu^{\prime} \in\{(0,3),(0,4),(0,5),(0,6)\}$, because $\sigma_{\text {Reg, } 1}=\rho_{2}=\{0\} \times \mathbb{R}_{\geq 0}$. By Proposition 4.19] only $\nu=(1,0)$ contributes to $F_{6}$ from the vectors in $H_{6}$, and the claim on $F_{6}$ follows. Note how at this level $\nu=(0,1)$ does no longer give rise to an irreducible component, since $l_{2}(0,1)=6$ and $(0,1) \notin N_{2}$. Then we have that $(0,2) \in F_{6}$ and the vertex associated with $C_{5}^{(0,1)}$ and the one associated with $C_{6}^{(0,2)}$ are joined in the graph $\Gamma^{\prime}$.

\section{TECHNICAL RESUltS AND PROOFS.}

In this section we state and prove some results which are used along the paper but only in the proofs of other results, and can be skipped to read Section 4. Moreover there are some proofs which we leave to this section.

Recall that we denote the first characteristic exponent by $\lambda_{1}=\gamma_{1}=\left(\frac{a_{1}}{n_{1}}, \frac{b_{1}}{n_{1}}\right)$ with $a_{1} \geq b_{1}, a_{1}>0$ and $b_{1} \geq$ 0 , and if $b_{1}=0$ then $a_{1}>n_{1}$ (we always consider normalized branches). If $g_{1}>0$ then, by Lemma 3.12, we have that $n_{g_{1}+1} \gamma_{g_{1}+1}=\left(\alpha_{1}^{\left(g_{1}+1\right)}, \alpha_{2}^{\left(g_{1}+1\right)}\right)+\left(r_{1}^{\left(g_{1}+1\right)} \gamma_{1}^{(1)}+\cdots+r_{g_{1}}^{\left(g_{1}+1\right)} \gamma_{g_{1}}^{(1)}, 0\right)$, therefore $n_{g_{1}+1} \gamma_{g_{1}+1}^{(2)} \in \mathbb{Z}$, or in other words, $\gamma_{g_{1}+1}=\left(\gamma_{g_{1}+1}^{(1)}, \frac{b_{g_{1}+1}}{n_{g_{1}+1}}\right)$ with $b_{g_{1}+1} \geq 1$.

Lemma 5.1. In the relation $n_{g_{2}+1} \gamma_{g_{2}+1}=\left(\alpha_{1}^{\left(g_{2}+1\right)}, \alpha_{2}^{\left(g_{2}+1\right)}\right)+r_{1}^{\left(g_{2}+1\right)} \gamma_{1}+\cdots+r_{g_{2}}^{\left(g_{2}+1\right)} \gamma_{g_{2}}$ given in Lemma 3.7, we have that $\alpha_{2}^{\left(g_{2}+1\right)}>1$.

Proof. If $g_{2}=g_{1}$ the claim is trivial since $\alpha_{2}^{\left(g_{2}+1\right)}=b_{g_{2}+1}>1$. Otherwise $n_{g_{2}+1} \gamma_{g_{2}+1}^{(2)}=\alpha_{2}^{\left(g_{2}+1\right)}+$ $r_{g_{2}}^{\left(g_{2}+1\right)} \frac{1}{n_{g_{2}}}$, and since, by Lemma 3.7. $\gamma_{g_{2}+1}^{(2)} \geq n_{g_{2}} \gamma_{g_{2}}^{(2)}=1$ and $0 \leq r_{g_{2}}^{\left(g_{2}+1\right)}<n_{g_{2}}$, then $\alpha_{2}^{\left(g_{2}+1\right)} \geq n_{g_{2}+1}-$ $\frac{r_{g_{2}}^{\left(g_{2}+1\right)}}{n_{g_{2}}}>1$, because $n_{g_{2}+1} \geq 2$.

In Corollary 4.15 we describe the generators of $J_{m}^{\nu}$ for $\nu \in H_{m} \cup L_{m}$. But we also need to describe the polynomial $F_{\nu}^{\left(l_{i(\nu)}(\nu)\right)}$ (recall that by definition $\nu \notin H_{l_{i(\nu)}(\nu)} \cup L_{l_{i(\nu)}(\nu)}$ ). We do this in the next Lemma, but before we look at an example.

Example 5.2. Let $X$ be a quasi-ordinary surface defined by $f=\left(\left(z^{2}-x_{1}^{3} x_{2}^{2}\right)^{2}-x_{1}^{6} x_{2}^{4} z\right)^{3}-x_{1}^{23} x_{2}^{14} z$. The generators of the semigroup are $\gamma_{1}=\left(\frac{3}{2}, 1\right), \gamma_{2}=\left(\frac{15}{4}, \frac{5}{2}\right)$ and $\gamma_{3}=\left(\frac{49}{6}, 5\right)$. Notice that $\nu=(0,3) \notin N_{2}$, and $l_{2}(\nu)=l_{3}(\nu)$. At level $m=45$ we have the set

$$
D_{45}^{(0,3)}=V\left(x_{2}^{(0)}, x_{2}^{(1)}, x_{2}^{(2)}, z^{(0)}, z^{(1)}, z^{(2)}, F_{1, \nu}^{(6)}, F_{1, \nu}^{(7)}, F_{3, \nu}^{(45)}\right) \cap D\left(x_{1}^{(0)}\right) \cap D\left(x_{2}^{(3)}\right),
$$

where

$$
\begin{aligned}
F_{3, \nu}^{(45)} & =F_{2, \nu}^{(15)^{3}}-x_{1}^{(0)^{23}} x_{2}^{(3)^{14}} z^{(3)} \\
& =\left(x_{1}^{(0)^{6}} x_{2}^{(3)^{4}} z^{(3)}\right)^{3}-x_{1}^{(0)}{ }^{23} x_{2}^{(3)}{ }^{14} z^{(3)} \\
= & \left(x_{1}^{(0)^{6}} x_{2}^{(3)^{4}} z^{(3)}\right)^{3}\left(1-\frac{x_{1}^{(0)} x_{2}^{(3)^{3}}}{z^{(3)^{2}}}\right),
\end{aligned}
$$

since $D_{45}^{(0,3)} \subset D\left(z^{(3)}\right)$. Since $\gamma(0)=\left(x_{1}^{(0)}, 0,0\right) \in X$, and we are considering germs of quasi-ordinary singularities, we have that $\left|x_{1}^{(0)}\right|<<1$ and we deduce that $1-\frac{x_{1}^{(0)^{8}} x_{2}^{(3)^{3}}}{z^{(3)^{2}}} \neq 0$.

This example illustrates the fact that we are looking at jet schemes of a germ of quasi-ordinary singularity, instead of jet schemes of the whole affine surface. If we looked at the whole surface there would be other irreducible components that we do not consider here. This is expectable because the components we consider are determined by the invariants of the topological type at the origin, so they describe only what happens in a small neighbourhood of zero. Actually the other components that may appear when looking at the whole affine surface, will project on closed points, different from the origin, of the singular locus. 
Lemma 5.3. Given $m \in \mathbb{Z}_{>0}$ and $\nu \in H_{m} \cup L_{m}$ with $m+1=l_{i(\nu)}(\nu)$, then

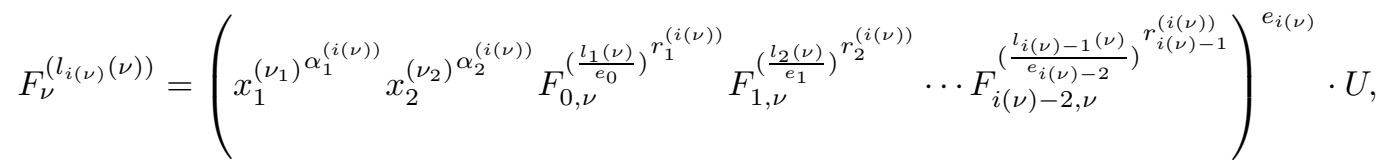

where $U$ is a unit in $\mathbb{C}\left[\left[x_{1}^{\left(\nu_{1}\right)^{ \pm 1}}, x_{2}^{\left(\nu_{2}\right)^{ \pm 1}}, F_{0}^{\left(\frac{l_{1}(\nu)}{e_{0}}\right)^{ \pm 1}}, \ldots, F_{i(\nu)-2}^{\left(\frac{l_{i(\nu)-1}(\nu)}{e_{(i \nu)-2}}\right)}\right]\right]$. When $\nu \notin \rho_{1} \cup \rho_{2}$, then $U=1$.

Proof. We have that $j(m, \nu)=i(\nu)-1$, and, by Lemma 4.14, for any $\gamma \in D_{m}^{\nu}$

$$
\begin{aligned}
& \operatorname{ord}_{t}\left(f_{k} \circ \gamma\right)=\left\langle\nu, \gamma_{k+1}\right\rangle \quad \text { for } 0 \leq k \leq i(\nu)-2 \\
& \operatorname{ord}_{t}\left(f_{i(\nu)-1} \circ \gamma\right)>\frac{m}{e_{i(\nu)-1}}
\end{aligned}
$$

Then $\operatorname{ord}_{t}\left(f_{i(\nu)-1} \circ \gamma\right) \geq \frac{m+1}{e_{i(\nu)-1}}=\frac{l_{i(\nu)}(\nu)}{e_{i(\nu)-1}}=\left\langle\nu, \gamma_{i(\nu)}\right\rangle$, and since $\nu \notin N_{i(\nu)},\left\langle\nu, \gamma_{i(\nu)}\right\rangle$ is not an integer. Hence

$$
\operatorname{ord}_{t}\left(f_{i(\nu)-1} \circ \gamma\right)>\left\langle\nu, \gamma_{i(\nu)}\right\rangle
$$

We have by Lemma 3.12

$$
f_{i(\nu)}=f_{i(\nu)-1}^{n_{i(\nu)}}-c_{i(\nu)} x_{1}^{\alpha_{1}^{(i(\nu))}} x_{2}^{\alpha_{2}^{(i(\nu))}} f_{0}^{r_{1}^{(i(\nu))}} \cdots f_{i(\nu)-2}^{r_{i(\nu)-1}^{(i(\nu))}}+\sum c_{\underline{\alpha}, \underline{r}} x_{1}^{\alpha_{1}} x_{2}^{\alpha_{2}} f_{0}^{r_{1}} \cdots f_{i(\nu)-1}^{r_{i(\nu)}},
$$

and

$$
\begin{aligned}
\operatorname{ord}_{t}\left(f_{i(\nu)-1}^{n_{i(\nu)}} \circ \gamma\right) & >n_{i(\nu)}\left\langle\nu, \gamma_{i(\nu)}\right\rangle \\
\operatorname{ord}_{t}\left(\left(c_{i(\nu)} x_{1}^{\alpha_{1}^{(i(\nu))}} x_{2}^{\alpha_{2}^{(i(\nu))}} \cdots f_{i(\nu)-2}^{r_{i(\nu))}^{(i(\nu))}}\right) \circ \gamma\right) & =n_{i(\nu)}\left\langle\nu, \gamma_{i(\nu)}\right\rangle \\
\operatorname{ord}_{t}\left(\left(c_{\underline{\alpha}, \underline{r}} x_{1}^{\alpha_{1}} x_{2}^{\alpha_{2}} \cdots f_{i(\nu)-1}^{r_{i(\nu)}}\right) \circ \gamma\right) & =\left\langle\nu,\left(\alpha_{1}, \alpha_{2}\right)+r_{1} \gamma_{1}+\cdots+r_{i(\nu)-1} \gamma_{i(\nu)-1}\right\rangle+r_{i(\nu)} \operatorname{ord}_{t}\left(f_{i(\nu)-1} \circ \gamma\right) \\
& >\left\langle\nu,\left(\alpha_{1}, \alpha_{2}\right)+r_{1} \gamma_{1}+\cdots+r_{i(\nu)} \gamma_{i(\nu)}\right\rangle \\
& \geq n_{i(\nu)}\left\langle\nu, \gamma_{i(\nu)}\right\rangle .
\end{aligned}
$$

Then $\operatorname{ord}_{t}\left(f_{i(\nu)} \circ \gamma\right)=n_{i(\nu)}\left\langle\nu, \gamma_{i(\nu)}\right\rangle=\frac{l_{i(\nu)}(\nu)}{e_{i(\nu)}}$, and

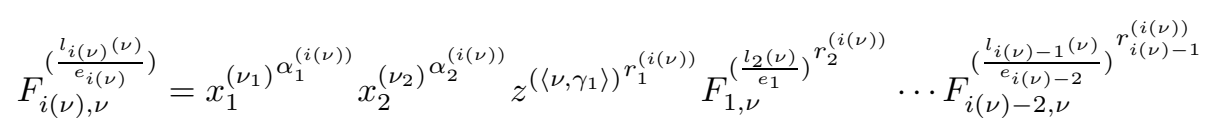

By Lemma 3.13

$$
f=f_{i(\nu)}^{e_{i(\nu)}}+\sum c_{i j k}^{(i(\nu))} x_{1}^{i} x_{2}^{j} z^{k}
$$

where $(i, j)+k \gamma_{1}>n_{i(\nu)} e_{i(\nu)} \gamma_{i(\nu)}$. Then

$$
\begin{aligned}
& \operatorname{ord}_{t}\left(f_{i(\nu)}^{e_{i(\nu)}} \circ \gamma\right)=l_{i(\nu)}(\nu) \\
& \operatorname{ord}_{t}\left(\left(c_{i j k}^{(i(\nu))} x_{1}^{i} x_{2}^{j} z^{k}\right) \circ \gamma\right) \geq l_{i(\nu)}(\nu)
\end{aligned}
$$

and hence

$$
F_{\nu}^{\left(l_{i(\nu)}(\nu)\right)}=F_{i(\nu), \nu}^{\left(\frac{l_{i(\nu)}(\nu)}{e_{i(\nu)}}\right)^{e_{i(\nu)}}}+G_{i(\nu), \nu}
$$

where

$$
G_{i(\nu), \nu}=\sum c_{i j k}^{(i(\nu))} x_{1}^{\left(\nu_{1}\right)^{i}} x_{2}^{\left(\nu_{2}\right)^{j}} z^{\left(\left\langle\nu, \gamma_{1}\right\rangle\right)^{k}}
$$

and the sum runs over $i, j, k$ such that

(i) $c_{i j k}^{(i(\nu))} \neq 0$

(ii) $\left\langle\nu,(i, j)+k \gamma_{1}\right\rangle=l_{i(\nu)}(\nu)$ 
Notice that if $\nu \notin \rho_{1} \cup \rho_{2}$, then condition (ii) never holds and $G_{\nu, i(\nu)}=0$. In this case we are done. Otherwise, from (19) we deduce that $\gamma \in D_{m}^{\nu} \subset D\left(F_{\nu, i(\nu)}^{\left.\frac{\left(l_{i(\nu)}(\nu)\right.}{e_{i(\nu)}}\right)}\right)$, and hence

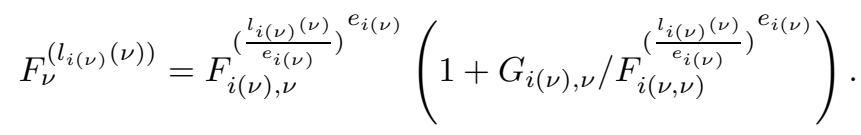

Proof of Lemma 4.8. If $m<l_{i(\nu)}(\nu)$, then we deduce from Proposition 4.16 that $D_{m}^{\nu}$ is non-empty. Otherwise $m \geq l_{i(\nu)}(\nu)$, and by definition $F^{\left(l_{i(\nu)}(\nu)\right)} \in J_{m}^{\nu}$, and by Lemma 5.3 (and its proof)

$$
F_{\nu}^{\left(l_{i(\nu)}(\nu)\right)}=F_{i_{(\nu), \nu}}^{\left(\frac{l_{i(\nu)}(\nu)}{e_{i(\nu)}}\right)^{e_{i(\nu)}}} \cdot U
$$

where

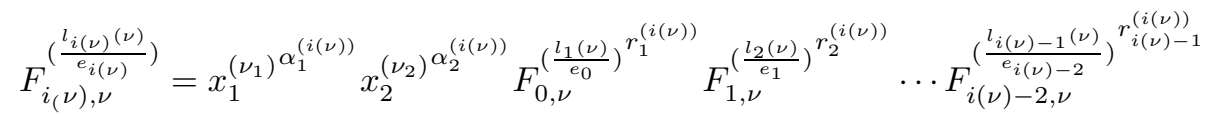

with $\left(\alpha_{1}^{(i(\nu))}, \alpha_{2}^{(i(\nu))}\right)+r_{1}^{(i(\nu))} \gamma_{1}+\cdots+r_{i(\nu)-1}^{(i(\nu))} \gamma_{i(\nu)-1}=n_{i(\nu)} \gamma_{i(\nu)}$. And

$$
U=1+G_{i(\nu), \nu} / F_{i(\nu), \nu}^{\left(\frac{l_{i(\nu)}(\nu)}{e_{i(\nu)}}\right)^{e_{i(\nu)}}}
$$

where

$$
G_{i(\nu), \nu}=\sum c_{i j k}^{(i(\nu))} x_{1}^{\left(\nu_{1}\right)^{i}} x_{2}^{\left(\nu_{2}\right)^{j}} z^{\left(\left\langle\nu, \gamma_{1}\right\rangle\right)^{k}}
$$

with $\left\langle\nu,(i, j)+k \gamma_{1}\right\rangle=n_{i(\nu)} e_{i(\nu)}\left\langle\nu, \gamma_{i(\nu)}\right\rangle$, though $(i, j)+k \gamma_{1}>n_{i(\nu)} e_{i(\nu)} \gamma_{i(\nu)}$.

Notice that at level $l_{i(\nu)}(\nu)-1$ we can apply Corollary 4.15, and deduce, as in (19), that

$$
D_{l_{i(\nu)-1}(\nu)-1}^{\nu} \subseteq D\left(F_{0, \nu}^{\left(\frac{l_{1}(\nu)}{e_{0}}\right)} \cdots F_{i(\nu)-2, \nu}^{\left(\frac{l_{i(\nu)-1}(\nu)}{e_{i(\nu)-2}}\right)}\right)
$$

Hence $D_{l_{i(\nu)}(\nu)}^{\nu}$ satisfies the same property, and since, by definition, $D_{l_{i(\nu)}(\nu)}^{\nu} \subseteq D\left(x_{1}^{\left(\nu_{1}\right)} \cdot x_{2}^{\left(\nu_{2}\right)}\right)$, we just have to argue that $U \neq 0$.

In Lemma 5.3 we prove that $U=1$ when $\nu \notin \rho_{1} \cup \rho_{2}$. Suppose the contrary, then either $\nu_{1}=0$ or

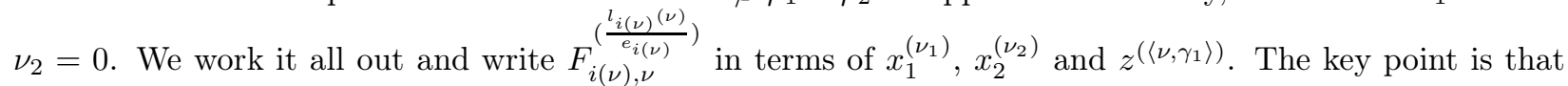
when $G_{i(\nu), \nu} \neq 0$ is because $(i, j)+k \gamma_{1}>n_{i(\nu)} e_{i(\nu)} \gamma_{i(\nu)}$ but $\left\langle\nu,(i, j)+k \gamma_{1}\right\rangle=n_{i(\nu)} e_{i(\nu)}\left\langle\nu, \gamma_{i(\nu)}\right\rangle$, because

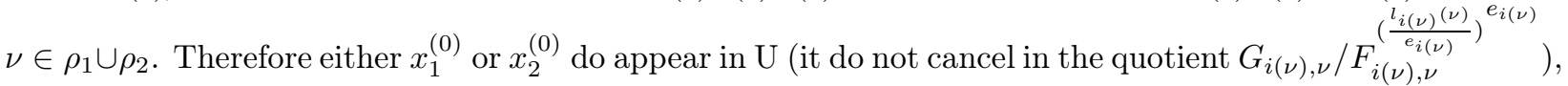
depending on whether $\nu_{1}=0$ or $\nu_{2}=0$. Then we have that $U$ depends on the origin of the jet $\gamma(0) \in X$, and since we are dealing with germs of quasi-ordinary singularities, we have that $\left|x_{i}^{(0)}\right|<<1$ for $i=1,2$, and we deduce that $U \neq 0$.

Proof of Lemma 4.14. We distinguish the cases $\nu \in H_{m}$ and $\nu \in L_{m}$. For $\nu \in H_{m}, j(m, \nu)=0$ and we have to prove that ord $\mathrm{d}_{t}\left(f_{k} \circ \gamma\right)>\frac{m}{e_{k}}$ for $0 \leq k \leq g$. By Proposition 4.12 it is true for $k=0$. For $k=g$ the claim is obvious, and for $1 \leq k \leq g-1$ we use the expansion (11) in Lemma 3.13,

$$
f=f_{k}^{e_{k}}+\sum_{(i, j)+r \gamma_{1}>n_{k} e_{k} \gamma_{k}} c_{i j r}^{(k)} x_{1}^{i} x_{2}^{j} z^{r} .
$$

Suppose that there exist $c_{i j r}^{(k)} \neq 0$ such that $\langle\nu,(i, j)\rangle+r \operatorname{ord}_{t}(z \circ \gamma) \leq m$. Then, using that $\operatorname{ord}_{t}(z \circ \gamma)>\frac{m}{n}$ and $k \geq 1$, we have the following inequalities

$$
l_{1}(\nu)-r\left\langle\nu, \gamma_{1}\right\rangle+r \frac{m}{n}<l_{1}(\nu)-r\left\langle\nu, \gamma_{1}\right\rangle+r \operatorname{ord}_{t}(z \circ \gamma) \leq l_{k}(\nu)-r\left\langle\nu, \gamma_{1}\right\rangle+r \operatorname{ord}_{t}(z \circ \gamma) \leq m .
$$

Then $l_{1}(\nu)-r\left\langle\nu, \gamma_{1}\right\rangle<m\left(1-\frac{r}{n}\right)$, and since $r<n$ this is equivalent to $\left\langle\nu, \gamma_{1}\right\rangle<\frac{m}{n}$, which contradicts the fact that $j(m, \nu)=0$. Hence $\operatorname{ord}_{t}\left(c_{i j r}^{(k)} x_{1}^{i} x_{2}^{j} z^{r} \circ \gamma\right)>m$ and therefore $\operatorname{ord}_{t}\left(f_{k}^{e_{k}} \circ \gamma\right)>m$ as we wanted to prove. 
For $\nu \in L_{m}$ the proof is by induction on $j(m, \nu)$. And we will make use repeatedly of the following observation. In general, for any function $f \in \mathbb{C}\left[x_{1}, x_{2}, z\right]$, and any $m$-jet $\gamma$, there is no relation among $\operatorname{ord}_{t}(f \circ \gamma)$ and $\operatorname{ord}_{t}\left(f \circ \pi_{m, m^{\prime}}(\gamma)\right)$ with $m^{\prime}<m$. But if $\operatorname{ord}_{t}\left(x_{i} \circ \gamma\right) \neq 0$ for $i=1,2, \operatorname{ord}_{t}(z \circ \gamma) \neq 0$, and for $m^{\prime}<m$, the $m^{\prime}$-jet $\gamma^{\prime}:=\pi_{m, m^{\prime}}(\gamma)$ verifies that $\operatorname{ord}_{t}\left(x_{i} \circ \gamma^{\prime}\right) \neq 0$ and $\operatorname{ord}_{t}\left(z \circ \gamma^{\prime}\right) \neq 0$, then

$$
\operatorname{ord}_{t}(f \circ \gamma)=\operatorname{ord}_{t}\left(f \circ \gamma^{\prime}\right) \text {. }
$$

By Remark 4.11 (ii) the first case of induction is $j(m, \nu)=1$. Then in particular $l_{1}(\nu)<l_{2}(\nu)$. For $\gamma \in D_{m}^{\nu}$, set $\bar{m}:=l_{1}(\nu)-1<m$ and $\bar{\gamma}:=\pi_{m, \bar{m}}(\gamma)$. Then $j(\bar{m}, \nu)=0$ and $\bar{\gamma} \in D_{\bar{m}}^{\nu}$. By Proposition 4.12 we have

$$
\operatorname{ord}_{t}(z \circ \bar{\gamma})>\frac{\bar{m}}{n}=\frac{l_{1}(\nu)-1}{n}=\left\langle\nu, \gamma_{1}\right\rangle-\frac{1}{n}
$$

Hence $\operatorname{ord}_{t}(z \circ \bar{\gamma}) \geq\left\langle\nu, \gamma_{1}\right\rangle$ and therefore $\operatorname{ord}_{t}(z \circ \gamma) \geq\left\langle\nu, \gamma_{1}\right\rangle$. Suppose that the inequality is strict, $\operatorname{ord}_{t}(z \circ \gamma)>$ $\left\langle\nu, \gamma_{1}\right\rangle$. By Lemma 3.13

Then

$$
f_{1}=z^{n_{1}}-x_{1}^{a_{1}} x_{2}^{b_{1}}+\sum_{\left(i_{1}, i_{2}\right)+k \gamma_{1}>n_{1} \gamma_{1}} x_{1}^{i_{1}} x_{2}^{i_{2}} z^{k}
$$

$$
\begin{aligned}
\operatorname{ord}_{t}\left(z^{n_{1}} \circ \gamma\right) & >n_{1}\left\langle\nu, \gamma_{1}\right\rangle \\
\operatorname{ord}_{t}\left(x_{1}^{a_{1}} x_{2}^{b_{1}} \circ \gamma\right) & =n_{1}\left\langle\nu, \gamma_{1}\right\rangle \\
\operatorname{ord}_{t}\left(x_{1}^{i_{1}} x_{2}^{i_{2}} z^{k} \circ \gamma\right) & >\left\langle\nu,\left(i_{1}, i_{2}\right)+k \gamma_{1}\right\rangle \geq n_{1}\left\langle\nu, \gamma_{1}\right\rangle
\end{aligned}
$$

and hence $\operatorname{ord}_{t}\left(f_{1} \circ \gamma\right)=n_{1}\left\langle\nu, \gamma_{1}\right\rangle$. Again by Lemma 3.13 we have

and since

$$
f=f_{1}^{e_{1}}+\sum_{\left(i_{1}, i_{2}\right)+k \gamma_{1}>n_{1} e_{1} \gamma_{1}} c_{i_{1} i_{2} k} x_{1}^{i_{1}} x_{2}^{i_{2}} z^{k}
$$

$$
\begin{aligned}
\operatorname{ord}_{t}\left(f_{1}^{e_{1}} \circ \gamma\right) & =e_{1} n_{1}\left\langle\nu, \gamma_{1}\right\rangle=l_{1}(\nu) \\
\operatorname{ord}_{t}\left(x_{1}^{i_{1}} x_{2}^{i_{2}} z^{k} \circ \gamma\right) & >\left\langle\nu,\left(i_{1}, i_{2}\right)+k \gamma_{1}\right\rangle \geq n_{1} e_{1}\left\langle\nu, \gamma_{1}\right\rangle=l_{1}(\nu)
\end{aligned}
$$

we deduce $\operatorname{ord}_{t}(f \circ \gamma)=l_{1}(\nu) \leq m$, which is a contradiction. Then $\operatorname{ord}_{t}(z \circ \gamma)=\left\langle\nu, \gamma_{1}\right\rangle$.

Now we prove that $\operatorname{ord}_{t}\left(f_{1} \circ \gamma\right)>\frac{m}{e_{1}}$. Suppose the contrary, $\operatorname{ord}_{t}\left(f_{1} \circ \gamma\right) \leq \frac{m}{e_{1}}$. If $g=1$ there is nothing to prove. If $g=2$, then we consider the expansion given in Lemma 3.12

$$
f=f_{2}=f_{1}^{n_{2}}-c_{2} x_{1}^{\alpha_{1}^{(2)}} x_{2}^{\alpha_{2}^{(2)}} z^{r_{1}^{(2)}}+\sum c_{\underline{\alpha}, \underline{r}} x_{1}^{i_{1}} x_{2}^{i_{2}} z^{r_{1}} f_{1}^{r_{2}}
$$

where $\left(i_{1}, i_{2}\right)+r_{1} \gamma_{1}+r_{2} \gamma_{2}>n_{2} \gamma_{2}$, and

$$
\begin{aligned}
\operatorname{ord}_{t}\left(f_{1}^{n_{2}} \circ \gamma\right) & \leq m, \\
\operatorname{ord}_{t}\left(x_{1}^{\alpha_{1}^{(2)}} x_{2}^{\alpha_{2}^{(2)}} z^{r_{1}^{(2)}} \circ \gamma\right) & =n_{2}\left\langle\nu, \gamma_{2}\right\rangle=l_{2}(\nu)>m
\end{aligned}
$$

Then there must exist $c_{\underline{\alpha}, \underline{r}} \neq 0$ such that

$$
n_{2} \operatorname{ord}_{t}\left(f_{1} \circ \gamma\right)=\left\langle\nu,\left(i_{1}, i_{2}\right)+r_{1} \gamma_{1}\right\rangle+r_{2} \operatorname{ord}_{t}\left(f_{1} \circ \gamma\right)
$$

or equivalently

$$
\left(n_{2}-r_{2}\right) \operatorname{ord}_{t}\left(f_{1} \circ \gamma\right)=\left\langle\nu,\left(i_{1}, i_{2}\right)+r_{1} \gamma_{1}\right\rangle \geq\left(n_{2}-r_{2}\right)\left\langle\nu, \gamma_{2}\right\rangle
$$

And since $r_{2}<n_{2}$ we conclude $\operatorname{ord}_{t}\left(f_{1} \circ \gamma\right) \geq\left\langle\nu, \gamma_{2}\right\rangle>\frac{m}{e_{1}}$, which is a contradiction.

If $g>2$, by Lemma 3.13

$$
f=f_{1}^{e_{1}}+\sum c_{\alpha, \underline{r}} x_{1}^{\alpha_{1}} x_{2}^{\alpha_{2}} z^{r_{1}} f_{1}^{r_{2}}
$$

and since we are supposing that $\operatorname{ord}_{t}\left(f_{1}^{e_{1}} \circ \gamma\right) \leq m$, there must exists $c_{\underline{\alpha}, \underline{r}} \neq 0$ such that $\operatorname{ord}_{t}\left(f_{1}^{e_{1}} \circ \gamma\right)=$ $\operatorname{ord}_{t}\left(x_{1}^{\alpha_{1}} x_{2}^{\alpha_{2}} z^{r_{1}} f_{1}^{r_{2}} \circ \gamma\right)$, hence

$$
e_{1} \operatorname{ord}_{t}\left(f_{1} \circ \gamma\right)=\left\langle\nu,\left(\alpha_{1}, \alpha_{2}\right)+r_{1} \gamma_{1}\right\rangle+r_{2} \operatorname{ord}_{t}\left(f_{1} \circ \gamma\right)
$$

or equivalently

$$
\left(e_{1}-r_{2}\right) \operatorname{ord}_{t}\left(f_{1} \circ \gamma\right)=\left\langle\nu,\left(\alpha_{1}, \alpha_{2}\right)+r_{1} \gamma_{1}\right\rangle \geq n_{2} e_{2}\left\langle\nu, \gamma_{2}\right\rangle-r_{2}\left\langle\nu, \gamma_{2}\right\rangle
$$

and since $r_{2}<e_{1}$ we conclude that $\operatorname{ord}_{t}\left(f_{1} \circ \gamma\right) \geq\left\langle\nu, \gamma_{2}\right\rangle$, which is a contradiction. 
The rest is simple, by Lemma 3.13 , for $k>1=j(m, \nu)$, we have

$$
f=f_{k}^{e_{k}}+\sum c_{i_{1} i_{2} k} x_{1}^{i_{1}} x_{2}^{i_{2}} z^{k}
$$

and since $\operatorname{ord}_{t}\left(c_{i_{1} i_{2} k} x_{1}^{i_{1}} x_{2}^{i_{2}} z^{k} \circ \gamma\right)=\left\langle\nu,\left(i_{1}, i_{2}\right)+k \gamma_{1}\right\rangle \geq l_{k}(\nu)>m$, we deduce $\operatorname{ord}_{t}\left(f_{k}^{e_{k}} \circ \gamma\right)>m$.

Suppose now that the claim is true for $j(m, \nu)=j$ and we will prove it for $j(m, \nu)=j+1$. Let $\gamma \in D_{m}^{\nu}$, with $l_{j+1}(\nu) \leq m<l_{j+1}(\nu)$. We set $\bar{m}=l_{j+1}(\nu)-1$ and $\bar{\gamma}=\pi_{m, \bar{m}}(\gamma)$. Then $\gamma \in D_{\bar{m}}^{\nu}$ and $j(\bar{m}, \nu)=i \leq j$, where

$$
l_{i}(\nu) \leq \bar{m}<l_{i+1}(\nu)=\cdots=l_{j+1}(\nu)
$$

Then, by Lemma 3.21, this is equivalent to

$$
\begin{gathered}
n_{i+1}\left\langle\nu, \gamma_{i+1}\right\rangle=\left\langle\nu, \gamma_{i+2}\right\rangle \\
n_{i+2}\left\langle\nu, \gamma_{i+2}\right\rangle=\left\langle\nu, \gamma_{i+3}\right\rangle \\
\vdots \\
n_{j}\left\langle\nu, \gamma_{j}\right\rangle=\left\langle\nu, \gamma_{j+1}\right\rangle
\end{gathered}
$$

By induction hypothesis we deduce $\operatorname{ord}_{t}\left(f_{k} \circ \gamma\right)=\operatorname{ord}_{t}\left(f_{k} \circ \bar{\gamma}\right)=\left\langle\nu, \gamma_{k+1}\right\rangle$ for $0 \leq k<i$. We are going to prove that $\operatorname{ord}_{t}\left(f_{i} \circ \gamma\right)=\left\langle\nu, \gamma_{i+1}\right\rangle$. By induction we have

$$
\operatorname{ord}_{t}\left(f_{i} \circ \gamma\right) \geq \operatorname{ord}_{t}\left(f_{i} \circ \bar{\gamma}\right)>\frac{\bar{m}}{e_{i}}=\left\langle\nu, \gamma_{i+1}\right\rangle-\frac{1}{e_{i}}
$$

Therefore

$$
\operatorname{ord}_{t}\left(f_{i} \circ \gamma\right) \geq\left\langle\nu, \gamma_{i+1}\right\rangle
$$

Let us suppose that $\left.\operatorname{ord}_{t}\left(f_{i} \circ \gamma\right)\right\rangle\left\langle\nu, \gamma_{i+1}\right\rangle$. By Lemma 3.13

$$
f_{i+1}=f_{i}^{n_{i+1}}-c_{i+1} x_{1}^{\alpha_{1}^{(i+1)}} x_{2}^{\alpha_{2}^{(i+1)}} z^{r_{1}^{(i+1)}} \cdots f_{i-1}^{r_{i}^{(i+1)}}+\sum c_{\underline{\alpha}, \underline{r}} x_{1}^{\alpha_{1}} x_{2}^{\alpha_{2}} z^{r_{1}} \cdots f_{i}^{r_{i+1}}
$$

and we have

$$
\begin{aligned}
\operatorname{ord}_{t}\left(f_{i}^{n_{i+1}} \circ \gamma\right) & >n_{i+1}\left\langle\nu, \gamma_{i+1}\right\rangle \\
\operatorname{ord}_{t}\left(x_{1}^{\alpha_{1}^{(i+1)}} x_{2}^{\alpha_{2}^{(i+1)}} z^{r_{1}^{(i+1)}} \circ \gamma\right) & =n_{i+1}\left\langle\nu, \gamma_{i+1}\right\rangle \\
\operatorname{ord}_{t}\left(c_{\underline{\alpha}, \underline{r}} x_{1}^{\alpha_{1}} x_{2}^{\alpha_{2}} z^{r_{1}} \cdots f_{i}^{r_{i+1}} \circ \gamma\right) & >\left\langle\nu,\left(\alpha_{1}, \alpha_{2}\right)+r_{1} \gamma_{1}+\cdots+r_{i+1} \gamma_{i+1}\right\rangle \geq n_{i+1}\left\langle\nu, \gamma_{i+1}\right\rangle
\end{aligned}
$$

Therefore $\operatorname{ord}_{t}\left(f_{i+1} \circ \gamma\right)=n_{i+1}\left\langle\nu, \gamma_{i+1}\right\rangle$. By Lemma 3.13

$$
f=f_{i+1}^{e_{i+1}}+\sum c_{\underline{\alpha}, \underline{r}} x_{1}^{\alpha_{1}} x_{2}^{\alpha_{2}} z^{r_{1}} \cdots f_{i}^{r_{i+1}}
$$

where $\left(\alpha_{1}, \alpha_{2}\right)+r_{1} \gamma_{1}+\cdots+r_{i+1} \gamma_{i+1}>n_{i+1} e_{i+1} \gamma_{i+1}$. Then

$$
\begin{aligned}
\operatorname{ord}_{t}\left(f_{i+1}^{e_{i+1}} \circ \gamma\right) & =l_{i+1}(\nu) \\
\operatorname{ord}_{t}\left(c_{\underline{\alpha}, \underline{r}} x_{1}^{\alpha_{1}} x_{2}^{\alpha_{2}} z^{r_{1}} \cdots f_{i}^{r_{i+1}} \circ \gamma\right) & >\left\langle\nu,\left(\alpha_{1}, \alpha_{2}\right)+r_{1} \gamma_{1}+\cdots+r_{i+1} \gamma_{i+1}\right\rangle \geq l_{i+1}(\nu)
\end{aligned}
$$

Then $\operatorname{ord}_{t}(f \circ \gamma)=l_{i+1}(\nu)=l_{j+1}(\nu) \leq m$, which is a contradiction. Therefore $\operatorname{ord}_{t}\left(f_{i} \circ \gamma\right)=\left\langle\nu, \gamma_{i+1}\right\rangle$.

We can prove that $\operatorname{ord}_{t}\left(f_{k} \circ \gamma\right)=\left\langle\nu, \gamma_{k+1}\right\rangle$ for $i<k \leq j$ one after the other exactly as the proof of $\operatorname{ord}_{t}\left(f_{i} \circ \gamma\right)=\left\langle\nu, \gamma_{i+1}\right\rangle$.

We prove now that $\operatorname{ord}_{t}\left(f_{j+1} \circ \gamma\right)>\frac{m}{e_{j+1}}$. Suppose the contrary, $\operatorname{ord}_{t}\left(f_{j+1} \circ \gamma\right) \leq \frac{m}{e_{j+1}}$.

If $j+2=g$ then by Lemma 3.13

$$
f=f_{j+2}=f_{j+1}^{n_{j+2}}-c_{j+2} x_{1}^{\alpha_{1}^{(g)}} x_{2}^{(g)} z^{r_{1}^{(g)}} \cdots f_{j}^{r_{j+1}^{(g)}}+\sum c_{\underline{\alpha}, \underline{r}} x_{1}^{\alpha_{1}} x_{2}^{\alpha_{2}} z^{r_{1}} \cdots f_{j+1}^{r_{j+2}}
$$

while, if $j+2<g$ we have the expansion

$$
f=f_{j+1}^{e_{j+1}}+\sum c_{\underline{\alpha}, \underline{r}} x_{1}^{\alpha_{1}} x_{2}^{\alpha_{2}} z^{r_{1}} \cdots f_{j+1}^{r_{j+2}}
$$

with $\left(\alpha_{1}, \alpha_{2}\right)+r_{1} \gamma_{1}+\cdots+r_{j+2} \gamma_{j+2}>n_{j+2} e_{j+2} \gamma_{j+2}$. In both cases we have

$$
f=f_{j+1}^{e_{j+1}}+\sum c_{\underline{\alpha}, \underline{r}} x_{1}^{\alpha_{1}} x_{2}^{\alpha_{2}} z^{r_{1}} \cdots f_{j+1}^{r_{j+2}}
$$


JET SCHEMES OF QUASI-ORDINARY SURFACE SINGULARITIES

with $\left(\alpha_{1}, \alpha_{2}\right)+r_{1} \gamma_{1}+\cdots+r_{j+2} \gamma_{j+2} \geq n_{j+2} e_{j+2} \gamma_{j+2}$. Looking at the expansion, since $\operatorname{ord}_{t}\left(f_{j+1}^{e_{j+1}} \circ \gamma\right) \leq m$, there must exist $c_{\underline{\alpha}, \underline{r}} \neq 0$ such that $\operatorname{ord}_{t}\left(f_{j+1}^{e_{j+1}} \circ \gamma\right)=\left\langle\nu,\left(\alpha_{1}, \alpha_{2}\right)+r_{1} \gamma_{1}+\cdots+r_{j+1} \gamma_{j+1}\right\rangle+r_{j+2} \operatorname{ord}_{t}\left(f_{j+1} \circ \gamma\right)$, or equivalently

$$
\left(e_{j+1}-r_{j+2}\right) \operatorname{ord}_{t}\left(f_{j+1} \circ \gamma\right)=\left\langle\nu,\left(\alpha_{1}, \alpha_{2}\right)+r_{1} \gamma_{1}+\cdots+r_{j+1} \gamma_{j+1}\right\rangle \geq n_{j+2} e_{j+2}\left\langle\nu, \gamma_{j+2}\right\rangle-r_{j+2}\left\langle\nu, \gamma_{j+2}\right\rangle
$$

And since $r_{j+2}<e_{j+1}$ we deduce that $\operatorname{ord}_{t}\left(f_{j+1} \circ \gamma\right) \geq\left\langle\nu, \gamma_{j+2}\right\rangle>\frac{m}{e_{j+1}}$, which is a contradiction.

Finally we prove that $\operatorname{ord}_{t}\left(f_{k}^{e_{k}} \circ \gamma\right)>m$. By Lemma 3.13, $f=f_{k}^{e_{k}}+\sum c_{i_{1} i_{2} r} x_{1}^{i_{1}} x_{2}^{i_{2}} z^{r}$ with $\left(i_{1}, i_{2}\right)+r \gamma_{1}>$ $n_{k} e_{k} \gamma_{k}$. Then $\operatorname{ord}_{t}\left(x_{1}^{i_{1}} x_{2}^{i_{2}} z^{r} \circ \gamma\right)=\left\langle\nu,\left(i_{1}, i_{2}\right)+r \gamma_{1}\right\rangle \geq l_{k}(\nu)>m$, and the result follows.

Lemma 5.4. For $m \in \mathbb{Z}_{>0}$ and $\nu \in L_{m}$, we have the following.

(i) If $i \leq j(m, \nu)$, then

$$
F_{i-1, \nu}^{\left(\frac{l_{i}(\nu)}{e_{i-1}}\right)}=0 \text { if and only if } x_{1}^{\left(\nu_{1}\right)^{\alpha_{1}^{(i)}}} x_{2}^{\left(\nu_{2}\right)^{\alpha_{2}^{(i)}}} z^{\left(\left\langle\nu, \gamma_{1}\right\rangle\right)^{r_{1}^{(i)}}} \cdots F_{i-2, \nu}^{\left(\frac{l_{i-1}(\nu)}{e_{i-2}}\right)^{r_{i-1}^{(i)}}}=0
$$

Roughly speaking the part $G_{i, \nu}$ in equation (18) is not meaningful.

(ii) For $m(\nu) \leq j \leq g_{1}$ we have

$$
V\left(I^{\nu}, F_{i, \nu}^{\left(\frac{l_{i}(\nu)}{e_{i}}\right)}\right)_{m(\nu) \leq i \leq j} \cap D\left(x_{1}^{\left(\nu_{1}\right)}\right) \subset D\left(z^{\left(\left\langle\nu, \gamma_{1}\right\rangle\right)}\right) \cap D\left(F_{1, \nu}^{\left(\frac{l_{2}(\nu)}{e_{1}}\right)}\right) \cap \cdots \cap D\left(F_{j-1, \nu}^{\left(\frac{l_{j}(\nu)}{e_{j-1}}\right)}\right),
$$

and for $g_{1}<j \leq g$ we have

$$
V\left(I^{\nu}, F_{i, \nu}^{\left(\frac{l_{i}(\nu)}{e_{i}}\right)}\right)_{m(\nu) \leq i \leq j} \cap D\left(x_{1}^{\left(\nu_{1}\right)}\right) \cap D\left(x_{2}^{\left(\nu_{2}\right)}\right) \subset D\left(z^{\left(\left\langle\nu, \gamma_{1}\right\rangle\right)}\right) \cap D\left(F_{1, \nu}^{\left(\frac{l_{2}(\nu)}{e_{1}}\right)}\right) \cap \cdots \cap D\left(F_{j-1, \nu}^{\left(\frac{l_{j}(\nu)}{e_{j}-1}\right)}\right) .
$$

\section{Proof.}

(i) First observe that if $j(m, \nu) \geq i$, then $F_{i, \nu}^{\left(\frac{l_{i}(\nu)}{e_{i}}\right)} \in J_{m}^{\nu}$ and it has the form given in equation (18) in Corollary 4.15. The proof is obvious if $G_{i, \nu}=0$. Suppose the contrary. Then $\nu \in \rho_{1} \cup \rho_{2}$, and the claim is not obvious if $\bar{G}_{i, \nu} \neq 0$, where

$$
\bar{G}_{i, \nu}=\sum c_{\underline{\alpha}, \underline{r}} x_{1}^{\left(\nu_{1}\right)^{\alpha_{1}}} x_{2}^{\left(\nu_{2}\right)^{\alpha_{2}}} z^{\left(\left\langle\nu, \gamma_{1}\right\rangle\right)^{r_{1}}} \cdots F_{i-2, \nu}^{\left(\frac{l_{i-1}(\nu)}{e_{i-2}}\right)^{r_{i-1}}}
$$

with the conditions $c_{\underline{\alpha}, \underline{r}} \neq 0,\left\langle\nu,\left(\alpha_{1}, \alpha_{2}\right)+r_{1} \gamma_{1}+\cdots+r_{i} \gamma_{i}\right\rangle=n_{i}\left\langle\nu, \gamma_{i}\right\rangle$ and $r_{i}=0$. Then, $F_{i-1, \nu}^{\left(\frac{l_{i}(\nu)}{e_{i-1}}\right)}=0$ if and only if

$$
c_{i} x_{1}^{\left(\nu_{1}\right)^{\alpha_{1}^{(i)}}} x_{2}^{\left(\nu_{2}\right)^{\alpha_{2}^{(i)}}} z^{\left(\left\langle\nu, \gamma_{1}\right\rangle\right)^{r_{1}^{(i)}}} \cdots F_{i-2, \nu}^{\left(\frac{l_{i-1}(\nu)}{e_{i-2}}\right)^{r_{i-1}^{(i)}}}+\bar{G}_{i, \nu}=0
$$

where, remember that any term $c_{\underline{\alpha}, \underline{\underline{r}}} x_{1}^{\left(\nu_{1}\right)^{\alpha_{1}}} x_{2}^{\left(\nu_{2}\right)^{\alpha_{2}}} z^{\left(\left\langle\nu, \gamma_{1}\right\rangle\right)^{r_{1}}} \cdots F_{i-2, \nu}^{\left(\frac{l_{i-1}(\nu)}{e_{i}-2}\right)^{r_{i-1}}}$ in particular appears in $F_{i, \nu}^{\left(\frac{l_{i}(\nu)}{e_{i}}\right)}$, and hence it satisfies that $\left(\alpha_{1}^{(i)}, \alpha_{2}^{(i)}\right)+r_{1} \gamma_{1}+\cdots+r_{i-1}^{(i)} \gamma_{i-1}<\left(\alpha_{1}, \alpha_{2}\right)+r_{1} \gamma_{1}+\cdots+r_{i-1} \gamma_{i-1}$. Then we can write last equation as

$$
x_{1}^{\left(\nu_{1}\right)^{\alpha_{1}^{(i)}}} x_{2}^{\left(\nu_{2}\right)^{\alpha_{2}^{(i)}}} z^{\left(\left\langle\nu, \gamma_{1}\right\rangle\right)^{r_{1}^{(i)}}} \cdots F_{i-2, \nu}^{\left(\frac{l_{i-1}(\nu)}{e_{i-2}}\right)^{r_{i-1}^{(i)}}}\left(c_{i}+P\left(x_{1}^{\left(\nu_{1}\right)}, x_{2}^{\left(\nu_{2}\right)}, z^{\left(\left\langle\nu, \gamma_{1}\right\rangle\right)}, \ldots, F_{i-2, \nu}^{\left(\frac{l_{i-1}(\nu)}{e_{i}-2}\right)}\right)\right)
$$

where $P$ is a polynomial non-unit. If we work it all out, then we can write $P$ as a polynomial in $x_{1}^{\left(\nu_{1}\right)}, x_{2}^{\left(\nu_{2}\right)}$ and $z^{\left(\left\langle\nu, \gamma_{1}\right\rangle\right)}$. Now we use that $\nu \in \rho_{1} \cup \rho_{2}$, and hence either $\nu_{1}=0$ or $\nu_{2}=0$. Then $P\left(x_{1}^{\left(\nu_{1}\right)}, x_{2}^{\left(\nu_{2}\right)}, z^{\left(\left\langle\nu, \gamma_{1}\right\rangle\right)}\right)$ depends on the origin $\gamma(0)$ of the jets, and, since we are dealing with germs of quasi-ordinary singularities we can always suppose that $\left|x_{i}^{\left(\nu_{i}\right)}\right|<<1$ for $i=1$ or 2 depending on whether $\nu_{1}=0$ or $\nu_{2}=0$. As a consequence we can always suppose that $P<<c_{i}$.

(ii) The inclusions follow directly from Corollary 4.15 when $G_{j, \nu}=0$. If $G_{j, \nu} \neq 0$, the proof is by induction on $j$. For $j=m(\nu)$ the claim says that

$$
V\left(I^{\nu}, F_{m(\nu), \nu}^{\left(\frac{l_{m(\nu)}(\nu)}{e_{m}(\nu)}\right)}\right) \cap D(M) \subset D\left(z^{\left(\left\langle\nu, \gamma_{1}\right\rangle\right)}\right),
$$


where

$$
M=\left\{\begin{array}{cc}
x_{1}^{\left(\nu_{1}\right)} & \text { if } g_{1} \geq 1 \\
x_{1}^{\left(\nu_{1}\right)} x_{2}^{\left(\nu_{2}\right)} & \text { if } g_{1}=0
\end{array}\right.
$$

and it follows by Lemma 4.14 Suppose it is true for $j-1$ and we prove it for $j$. We only have to prove that $F_{j-1, \nu}^{\left(\frac{l_{j}(\nu)}{e_{j-1}}\right)} \neq 0$. But this follows by (i).

\section{REFERENCES}

1. Abhyankar, S.S., On the ramification of algebraic functions. Amer. J. Math., 77. (1955), 575-592.

2. Aprodu, M., Naie, D., Enriques diagrams and log-canonical thresholds of curves on smooth surfaces. Geom. Dedicata 146 (2010), 43-66.

3. Artal Bartolo, E., Cassou-Noguès, Pi., Luengo, I. and Melle Hernández, A., Quasi-ordinary power series and their zeta functions. Mem. Amer. Math. Soc. 178 (2005), no. 841, 1-85.

4. Artal Bartolo, E., Cassou-Noguès, Pi., Luengo, I., Melle-Hernández, A., On the log-canonical threshold for germs of plane curves. (English summary) Singularities I, 1-14, Contemp. Math., 474, Amer. Math. Soc., Providence, RI, 2008.

5. Assi, A., Irreducibility criterion for quasi-ordinary polynomials. J. Singul. 4 (2012), 23-34.

6. Ban, C., McEwan, L., Canonical resolution of a quasi-ordinary surface singularity. Canad. J. Math. 52 (2000), no. 6, 1149-1163.

7. Ban, C., McEwan, L., Simultaneous resolution of equisingular quasi-ordinary singularities. Singularities in algebraic and analytic geometry (San Antonio, TX, 1999), 65-75, Contemp. Math., 266, Amer. Math. Soc., Providence, RI, 2000.

8. Budur, N., González Pérez, P.D. and González Villa, M., Log-canonical thresholds of quasi-ordinary hypersurfaces singularities. Proc. of the Amer. Math. Soc. 140 (2012) 4075-4083.

9. Cobo Pablos, H. and González Pérez, P.D., Geometric motivic Poincaré series of quasi-ordinary hypersurfaces, Math. Proc. Camb. Phil. Soc. 149 (2010), no. 01, 49-74.

10. Denef, J. and Loeser. F. Germs of arcs on singular algebraic varieties and motivic integration. Invent. Math. 135, 1, (1999), 201-232.

11. Denef, J. and Loeser. F. Geometry on arc spaces of algebraic varieties. European Congress of Mathematics, Vol. I (Barcelona, 2000), 327-348, Progr. Math., 201, Birkhäuser, Basel, 2001

12. Docampo, R., Arcs on determinantal varieties. Trans. Amer. Math. Soc. 365 (2013), no. 5, 2241-2269.

13. Ein, L. and Mustaţa, M. Jet Schemes and Singularities, Algebraic geometry-Seattle 2005, Proc. Sympos. Pure Math., 80, Part 2, Amer. Math. Soc., Providence, RI, 2009, 505-546.

14. Fulton, W. Introduction to toric varieties. Annals of Math. Studies (131), Princeton University Press, 1993.

15. García Barroso, E., Gwodziewicz, J., Quasi-ordinary singularities: tree model, discriminant and irreducibility. Int. Math. Res. Not., no. 14, (2015), 5783-5805.

16. Gau, Y-N., Embedded Topological classification of quasi-ordinary singularities, Memoirs of the American Mathematical Society $388,1988$.

17. González Pérez P.D., The semigroup of a quasi-ordinary hypersurface, J. Inst. Math. Jussieu, (2003), 2 (3), 383-399.

18. González Pérez, P.D., Toric embedded resolutions of quasi-ordinary hypersurface singularities, Ann. Inst. Fourier (Grenoble), 53 (6), (2003), 1819-1881.

19. González Pérez, P.D. and González Villa, M., Motivic Milnor fiber of a quasi-ordinary hypersurface. J. Reine Angew. Math. 687 (2014), 159-205.

20. Ishii, S., Jet schemes, arc spaces and the Nash problem. C. R. Math. Acad. Sci. Soc. R. Can. 29 (2007), no. 1, 1-21

21. Jung, H.W.E., Darstellung der Funktionen eines algebraischen Körpers zweier unabhaängigen Veränderlichen $x, y$ in der Umgebung einer stelle $x=a, y=b$, J.Reine Angew. Math., 133 (1908), 289-314.

22. Kiyek, K, Micus, M, Semigroup of a quasiordinary singularity. Topics in algebra, Part 2 (Warsaw, 1988), Banach Center Publ., 26, 149-156.

23. Lejeune-Jalabert, M., Mourtada, H., Reguera, A., Jet schemes and minimal embedded desingularization of plane branches. Revista de la Real Academia de Ciencias Exactas, Físicas y Naturales. Serie A. Matemáticas: 107, no. 1, (2013),145-157.

24. Lipman, J., Topological invariants of quasi-ordinary singularities, Memoirs of the American Mathematical Society, 74, no. $388,(1988), 1-107$.

25. Lipman, J., Equisingularity and simultaneous resolution of singularities. Resolution of singularities (Obergurgl, 1997), Progr. Math., 181, Birkhäuser, Basel, 2000, 485-505.

26. Mourtada, H., Jet schemes of complex plane branches and equisingularity, Annales de l'Institut Fourier, 61 (2011), no. 6, $2313-2336$

27. Mourtada, H., Jet schemes of toric surfaces. C. R. Math. Acad. Sci. Paris 349 (2011), no. 9-10, 563-566.

28. Mourtada, H., Jet schemes of normal toric surfaces. To appear in Bulletin de la SMF.

29. Mourtada, H., Jet schemes of rational double point surface singularities. Valuation Theory in Interaction, EMS Ser. Congr. Rep., Eur. Math. Soc., Sept. 2014, 373-388.

30. Mourtada, H., Jet schemes and minimal generating sequences of divisorial valuations in dimension 2. To appear at Michigan Math. Journal 
31. Mourtada, H., Plénat, C., Jet schemes and minimal toric embedded resolutions of rational double point singularities, arXiv: 1510.04894

32. Mustaţa, M., Singularities of pairs via jets schemes. J. Amer. Math. Soc. 15 (2002), no. 3, 599-615.

33. Mustata, M., The dimension of jet schemes of singular varieties. To appear in the Proceedings of the ICM, Seoul 2014. arXiv:1404.7731.

34. Popescu-Pampu, P., On the analytical invariance of the semigroups of a quasi-ordinary hypersurface singularity. Duke Math. J. 124 (2004), no. 1, 67-104.

35. Popescu-Pampu, P., Introduction to Jung's method of resolution of singularities. Contemporary Math. 538, (2011), 401-432.

36. Sethuraman, B. A.; Šivic, K., Jet schemes of the commuting matrix pairs scheme. Proc. Amer. Math. Soc. 137 (2009), no. 12, 3953-3967.

37. Teissier, B., Valuations, deformations, and toric geometry. Valuation theory and its applications, Vol. II (Saskatoon, SK, 1999), Fields Inst. Commun., 33, Amer. Math. Soc., Providence, RI, 2003, 361-459.

38. Veys, W., Arc spaces, motivic integration and stringy invariants, Advanced Studies in Pure Mathematics 43, Proceedings of "Singularity Theory and its applications, Sapporo (Japan), 16-25 september 2003" (2006), 529-572.

39. Villamayor, O., On equiresolution and a question of Zariski, Acta Math. 185 (2000) no.1, 123-159.

40. Yuen, C., Jet schemes of determinantal varieties. Algebra, geometry and their interactions, Contemp. Math., 448, Amer. Math. Soc., Providence, RI, 2007, 261-270.

E-mail address: helenacobo@gmail.com

Institut de Mathématiques de Jussieu-Paris Rive Gauche, Université Paris 7, Bâtiment Sophie Germain, 75013 Paris, France.

E-mail address: hussein.mourtada@imj-prg.fr 